Supporting Information

for

\title{
Studies Toward Australifungin. A Synthesis Dilemma of Regioselective Keto-Enol Tautomerization
}

\author{
David R. Williams, ${ }^{*, \dagger}$ J. Cullen Klein, ${ }^{\dagger}$ Lucas C. Kopel, ${ }^{\dagger}$ \\ Nhu Nguyen, ${ }^{\ddagger}$ and Dean J. Tantillo*,‡ \\ †Department of Chemistry, Indiana University \\ Bloomington, IN 47405, United States \\ williamd@indiana.edu \\ ‡Department of Chemistry, University of California, \\ Davis, California, 95616, United States \\ djtantillo@ucdavis.edu
}
Page
General Information
$S-2-S-3$
Experimental Procedures and Characterization Data
S-4-S-27
${ }^{1} \mathrm{H}$ and ${ }^{13} \mathrm{C}$ NMR Spectra
S-28-S-76
Computational Analysis
S-77 - S-89 


\section{Solvents and Reagents}

\section{General Methods}

Diethyl ether $\left(\mathrm{Et}_{2} \mathrm{O}\right)$ and tetrahydrofuran (THF) were distilled under argon from sodium-benzophenone ketyl immediately before use. Methylene chloride $\left(\mathrm{CH}_{2} \mathrm{Cl}_{2}\right)$ and toluene were distilled from calcium hydride. Hexanes and ethyl acetate (EtOAc) were distilled prior to their use for chromatography.

Triethylamine $\left(\mathrm{Et}_{3} \mathrm{~N}\right), N, N$-diisopropylethylamine $\left(i-\mathrm{Pr}_{2} \mathrm{NEt}\right)$, pyridine, and trimethylsilyl chloride were distilled from calcium hydride immediately prior to use. Cuprous iodide $(\mathrm{CuI})$ was continuously washed with ether over 2 days with a Soxhlet extractor, dried under vacuum and stored in the dark. Potassium tert-butoxide was sublimed and tert-butyl acetate was dried over $\mathrm{K}_{2} \mathrm{CO}_{3}$ and activated $4 \AA$ molecular sieves prior to use. Purification of meta-chloroperbenzoic acid was accomplished by dissolution in $\mathrm{CH}_{2} \mathrm{Cl}_{2}$, washing repeatedly with $\mathrm{pH} 7$ buffer ( 5 washes), and drying over anhydrous $\mathrm{Na}_{2} \mathrm{SO}_{4}$ before being concentrated to a solid which was then used without subsequent recrystallization. Tert-butanol was dried by gently stirring a slightly warmed volume over activated $3 \AA$ molecular sieves. Dess-Martin periodinane was prepared from 2iodobenzoic acid by oxidation to IBX according to Santagostino ${ }^{1}$ and then converted to the periodinane according to Ireland. ${ }^{2}$ All other reagents were used as provided by the chemical supplier. Reactions were typically carried out in flame or oven dried glassware under a positive atmosphere of dry argon.

\section{Chromatography}

Analytical thin layer chromatography (TLC) was performed on precoated $(0.25$ mm thickness) glass plates (E. Merck, silica gel 60F-254). Developed plates were visualized by UV light $(254 \mathrm{~nm})$ and stained with either an acidic ethanolic solution of $p$ anisaldehyde or an acidic solution of $\left(\mathrm{NH}_{4}\right)_{6} \mathrm{Mo}_{7} \mathrm{O}_{24} \cdot \mathrm{H}_{2} \mathrm{O}$ and ceric sulfate followed by heating. Preparative chromatography was performed using flash column chromatography with Kieselgel-60 (230-400 mesh) silica gel (E. Merck). Compounds were applied directly to silica gel and eluted under a positive pressure of in-house air. Removal of solvents from samples (in vacuo) was accomplished using a Büchi rotary-evaporator at aspirator pressure. All non-volatile compounds were evacuated to constant weight under high vacuum $(0.2-0.5 \mathrm{~mm} \mathrm{Hg})$ at room temperature.

\section{Physical Data}

Optical rotations were measured on a Perkin-Elmer 241 polarimeter at wavelength $589 \mathrm{~nm}$ (sodium, D-line) using a standard $10 \mathrm{~cm}$ cell ( $1 \mathrm{~mL}$ volume). Specific rotations, $[\alpha]_{D}^{\text {temp }}$, are reported in degree $\cdot \mathrm{mL} /(\mathrm{g} \cdot \mathrm{dm})$ at the specified temperature. Concentrations (c) are given in grams per $100 \mathrm{~mL}$ of the specified solvent. Proton $\left({ }^{1} \mathrm{H}-\mathrm{NMR}\right)$ and carbon $\left({ }^{13} \mathrm{C}-\mathrm{NMR}\right)$ nuclear magnetic resonance spectra were recorded on Varian Gemini 300, Varian VXR-400, Varian Inova-400 and Varian Inova-500 spectrometers. The chemical shifts are reported in parts per million $(\delta, \mathrm{ppm})$ downfield from tetramethylsilane (TMS) using residual $\mathrm{CHCl}_{3}(\delta 7.26 \mathrm{ppm})$ as an internal reference. Coupling constants are reported in Hertz $(\mathrm{Hz})$ and the data is presented in the following form: chemical shift (multiplicity, coupling constants, number of protons) for each resonance. Multiplicities are recorded by the following abbreviations: s, singlet; $\mathrm{d}$, doublet; $\mathrm{t}$, triplet; q, quartet; $\mathrm{m}$,

${ }^{1}$ Frigerio, M.; Santagostino, M.; Sputore, S. J. Org. Chem. 1999, 64, 4537-4538.

${ }^{2}$ Ireland, R. E.; Liu, L. J. Org. Chem. 1993, 58, 2899. 
multiplet; br, broad. All carbon chemical shifts are reported in $\delta$ using $\mathrm{CDCl}_{3}$ as an internal reference $(\delta 77.16 \mathrm{ppm})$. Infrared (IR) spectra were recorded on a Nicolet Avatar 360 FT-IR as a neat film or as the residue of a dichloromethane solution as indicated. Infrared spectral data are reported in wavenumbers $\left(\mathrm{cm}^{-1}\right)$ and calibrated to the $1601 \mathrm{~cm}^{-1}$ absorption of polystyrene film. High resolution mass spectra were recorded on a Thermo Electron MAT 95XP mass spectrometer by use of electron ionization (EI), chemical ionization (CI), or electrospray ionization (ESI). 


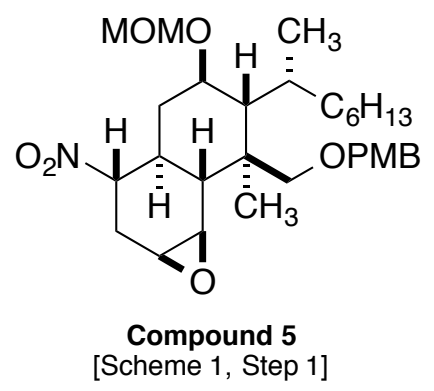

Epoxide 5 - To a solution of nitro-olefin 4 (477 $\mathrm{mg}, 0.912 \mathrm{mmol})$ and 4,4'-thiobis(6-tertbutyl- $m$-cresol) $(3.1 \mathrm{mg}, 0.027 \mathrm{mmol})$ in methylene chloride $(3.0 \mathrm{~mL})$ at $0{ }^{\circ} \mathrm{C}$ was added $m$-chloroperbenzoic acid (472 mg, $2.736 \mathrm{mmol}$ ) portionwise over $4.5 \mathrm{~h}$ (4 equal portions). The cooling bath was removed after the addition of the initial portion and the solution was allowed to come to room temperature. After the reaction was complete by TLC analysis (approx. $6 \mathrm{~h}$ ) anhydrous KF (318 mg, $5.47 \mathrm{mmol})$ was added and the heterogeneous mixture was stirred for $5 \mathrm{~min}$ before 1:1 ether-pentane $(12 \mathrm{~mL})$ was added and mixture then filtered through Celite. The filtrant was washed with 1:1 ether-pentane $(24 \mathrm{~mL})$ and the resulting filtrate was washed in a separatory funnel with $2 \times 50 \mathrm{~mL}$ of a solution of $5 \mathrm{~mL}$ saturated $\mathrm{Na}_{2} \mathrm{SO}_{3}, 50 \mathrm{~mL}$ of $\mathrm{pH} 7$ buffer and $45 \mathrm{~mL}$ of water. The aqueous layer was extracted with ether $(20 \mathrm{~mL})$ and the organic layers were combined, dried over anhydrous $\mathrm{Na}_{2} \mathrm{SO}_{4}$, filtered and concentrated in vacuo. Purification by flash chromatography (25 $\mathrm{g} \mathrm{SiO}_{2}$ ), eluting with gradient of 10:1-1:1 hexanes-ethyl acetate, provided epoxide 5 (411 $\mathrm{mg}, 0.770 \mathrm{mmol}, 84 \%$ ) as a colorless viscous oil (NMR shows 4 present in small amounts).

For epoxide 5:

$\mathrm{R}_{\mathrm{f}} 0.38$ (4:1 hexanes-EtOAc);

$[\alpha]_{\mathrm{D}}^{20}-11.4\left(c 0.62, \mathrm{CH}_{2} \mathrm{Cl}_{2}\right)$;

${ }^{1} \mathrm{H}-\mathrm{NMR}\left(400 \mathrm{MHz}, \mathrm{CDCl}_{3}\right) \delta$ 7.28-7.23 (m, 2H), 6.89-6.86 (m, 2H), 4.64-4.60 (m, 2H), 4.51-4.35 (m, 3H), $3.81(\mathrm{~s}, 3 \mathrm{H}), 3.64(\mathrm{td}, J=11.0,4.8 \mathrm{~Hz}, 1 \mathrm{H}), 3.50-3.32(\mathrm{~m}, 2 \mathrm{H})$, $3.31(\mathrm{~s}, 3 \mathrm{H}), 3.04(\mathrm{~d}, J=4.0 \mathrm{~Hz}, 1 \mathrm{H}), 2.71(\mathrm{ddd}, J=14.0,4.4,1.6 \mathrm{~Hz}, 1 \mathrm{H}), 2.32$ (ddd, $J=$ 13.6, 11.2, $2.0 \mathrm{~Hz}, 1 \mathrm{H}), 2.05-1.94(\mathrm{~m}, 2 \mathrm{H}), 1.82-1.71(\mathrm{~m}, 2 \mathrm{H}), 1.63-1.56(\mathrm{~m}, 1 \mathrm{H}), 1.46-$ $1.06(\mathrm{~m}, 12 \mathrm{H}), 1.04(\mathrm{~d}, J=7.2 \mathrm{~Hz}, 3 \mathrm{H}), 0.88(\mathrm{t}, J=6.8 \mathrm{~Hz}, 3 \mathrm{H}), 0.76(\mathrm{~s}, 3 \mathrm{H})$;

${ }^{13} \mathrm{C}-\mathrm{NMR}\left(100 \mathrm{MHz}, \mathrm{CDCl}_{3}\right) \delta 159.3,130.4,129.23,113.8,96.3,86.7,76.2,73.0,71.8$, 56.0, 55.4, 52.0, 50.3, 43.8, 42.7, 35.3, 35.2, 32.5, 32.2, 32.0, 30.7, 29.9, 28.9, 28.8, 22.9, $22.8,14.2$

IR (film) 3415 (br), 2954, 2924, 2853, 1612, 1586, 1551, 1513, 1463, 1377, 1032, 1248 , $1209,1172,1137,1097,1039,916,823 \mathrm{~cm}^{-1}$;

HRMS (CI+) calc. for $\mathrm{C}_{30} \mathrm{H}_{46} \mathrm{O}_{7} \mathrm{~N}[\mathrm{M}-\mathrm{H}]^{+}$532.3269. Found: 532.3260. 


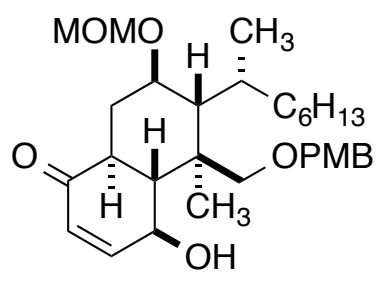

Compound 6

[Scheme 1, Step 2]

Enone 6: Epoxide 5 (499.7 mg, $0.936 \mathrm{mmol})$ was dissolved in dry THF (6.2 mL) and cooled to $10^{\circ} \mathrm{C}$. Solid potassium tert-butoxide $(157.6 \mathrm{mg}, 1.40 \mathrm{mmol})$ was added and the mixture was allowed to stir for 10 min during which the solution turned a bright yellow. A solution of dimethyldioxirane (freshly dried over anhydrous $\mathrm{Na}_{2} \mathrm{SO}_{4}$ ) was added in an amount until the solution turned colorless ( $\sim 37 \mathrm{~mL}$ of a $0.05 \mathrm{M}$ solution). The reaction mixture was allowed to stir for an additional $10 \mathrm{~min}$ at $10{ }^{\circ} \mathrm{C}$ before sequentially adding ether $(40 \mathrm{~mL})$, and a 1:1:1 solution of saturated $\mathrm{Na}_{2} \mathrm{~S}_{2} \mathrm{O}_{3}, \mathrm{pH} 7$ buffer, and water (total $30 \mathrm{~mL}$ ). The mixture was then stirred vigorously for $15 \mathrm{~min}$ and then poured into a separatory funnel. The layers separated and the aqueous layer back extracted with ether $(2 \times 40 \mathrm{~mL})$. The combined organic layers were then washed with saturated $\mathrm{NaHCO}_{3}$, dried over anhydrous $\mathrm{Na}_{2} \mathrm{SO}_{4}$, filtered and concentrated. The residue was then redissolved in dry $1: 1 \mathrm{THF}-t-\mathrm{BuOH}(10 \mathrm{~mL})$ and a cooled to $-10^{\circ} \mathrm{C}$ before adding a solution of potassium tert-butoxide in $1: 1 \mathrm{THF}-t$-BuOH $(1.5 \mathrm{~mL}, 0.1 \mathrm{M}, 0.15$ $\mathrm{mmol})$ dropwise. The mixture was allowed to stir until TLC indicated the more polar component was completely formed. Ethyl acetate $(50 \mathrm{~mL})$ and $\mathrm{pH} 7$ buffer $(50 \mathrm{~mL})$ were added and the layers separated. The aqueous layer was extracted with ethyl acetate (50 $\mathrm{mL})$ and the combined organic layers were then washed with water $(50 \mathrm{~mL})$ and brine $(50 \mathrm{~mL})$, dried over anhydrous $\mathrm{Na}_{2} \mathrm{SO}_{4}$, filtered and concentrated in vacuo. Purification by flash chromatography $\left(25 \mathrm{~g} \mathrm{SiO}_{2}\right.$ ) eluting with gradient of 16:1-1:1 hexanes-EtOac provided enone $6(373 \mathrm{mg}, 0.742 \mathrm{mmol}, 79 \%)$ as a clear colorless oil along with starting material $5(35.2 \mathrm{mg})$ and epi-5 $(27.8 \mathrm{mg})$.

For ketone 6:

$\mathrm{R}_{\mathrm{f}} 0.21(2: 1$ hexanes-EtOAc);

$[\alpha]_{\mathrm{D}}^{23}+33.8\left(c 0.87, \mathrm{CHCl}_{3}\right)$;

${ }^{1} \mathrm{H}-\mathrm{NMR}\left(500 \mathrm{MHz}, \mathrm{CDCl}_{3}\right) \delta 7.28-7.17(\mathrm{~m}, 2 \mathrm{H}), 6.90-6.82(\mathrm{~m}, 2 \mathrm{H}), 6.77(\mathrm{dd}, J=10.2$, $2.0 \mathrm{~Hz}, 1 \mathrm{H}), 5.91(\mathrm{dd}, J=10.1,1.9 \mathrm{Jz}, 1 \mathrm{H}), 4.71(\mathrm{~d}, J=8.9 \mathrm{~Hz}, 1 \mathrm{H}), 4.65-4.53(\mathrm{~m}, 2 \mathrm{H})$, 4.42 (dd, $J=22.2,11.0 \mathrm{~Hz}, 3 \mathrm{H}), 3.77$ (s, 3H), 3.70 (td, $J=9.7,4.2 \mathrm{~Hz}, 1 \mathrm{H}), 3.59$ (d, $J=$ $10.0 \mathrm{~Hz}, 1 \mathrm{H}), 3.52-3.45(\mathrm{~m}, 1 \mathrm{H}), 3.36(\mathrm{~s}, J=8.0 \mathrm{~Hz}, 3 \mathrm{H}), 2.69-2.56(\mathrm{~m}, 1 \mathrm{H}), 2.15-1.99$ $(\mathrm{m}, 2 \mathrm{H}), 1.60-1.48(\mathrm{~m}, 1 \mathrm{H}), 1.47-1.05(\mathrm{~m}, 12 \mathrm{H}), 0.99$ (d, $J=12.7 \mathrm{~Hz}, 3 \mathrm{H}), 0.91-0.82$ (m, 6H); 
${ }^{13} \mathrm{C}-\mathrm{NMR}\left(100 \mathrm{MHz}, \mathrm{CDCl}_{3}\right) \delta 199.4,159.6,153.0,129.8,129.0,126.8,114.0,95.6$, 75.2, 74.9, 73.1, 66.7, 56.1, 55.3, 53.2, 51.7, 43.4, 41.6, 32.6, 32.5, 32.0, 31.6, 29.9, 28.9, $22.7,22.6,14.2,13.1$;

IR (film) 3415 (br), 2954, 2929, 2872, 1678, 1612, 1513, 1465, 1382, 1302, 1248, 1101, $1040,820 \mathrm{~cm}^{-1}$;

HRMS (ES+) calc. for $\mathrm{C}_{30} \mathrm{H}_{46} \mathrm{O}_{6} \mathrm{Na}[\mathrm{M}+\mathrm{Na}]^{+}$525.3192. Found: 525.3199 . 


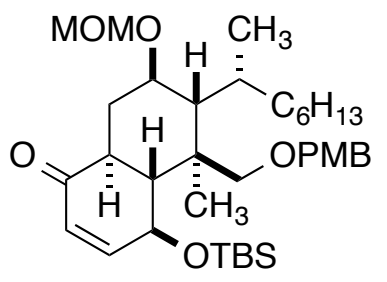

Compound 6a

[Scheme 1, Step 6]

TBS ether 6a: In a flame dried round-bottomed flask fitted with a magnetic stirbar was placed alcohol 6 (73.4 mg, $0.146 \mathrm{mmol})$ dissolved in dry $\mathrm{CH}_{2} \mathrm{Cl}_{2}(3 \mathrm{~mL})$. An argon atmosphere over the reaction mixture was generated and the reaction cooled to $-40{ }^{\circ} \mathrm{C}$. At this point 2,6 -lutidine $(70 \mu \mathrm{L}, 0.601 \mathrm{mmol})$ was added via syringe followed by TBSOTf ( $40 \mu \mathrm{L}, 0.174 \mathrm{mmol})$ which was also added via syringe. After a $4 \mathrm{~h}$, additional quantities of 2,6-lutidine $(70 \mu \mathrm{L}, 0.601 \mathrm{mmol})$ and TBSOTf $(40 \mu \mathrm{L}, 0.174 \mathrm{mmol})$ were added. After another $2 \mathrm{~h}$, the reaction mixture was quenched by the addition water $(6$ $\mathrm{mL}$ ) and the cooling bath removed. After approximately $15 \mathrm{~min}$ the mixture was poured into ether $(12 \mathrm{~mL})$. The layers were separated and the aqueous layer extracted with ether $(2 \times 6 \mathrm{~mL})$. The combined organic portion was washed with brine $(15 \mathrm{~mL})$, dried over anhydrous $\mathrm{MgSO}_{4}$, filtered and concentrated in vacuo. The residue was then purified by flash chromatography ( $5 \mathrm{~g} \mathrm{SiO}_{2}, 12: 1-4: 1$ hexanes-EtOAc) afforded silyl ether $\mathbf{6 a}(74.1$ $\mathrm{mg}, 0.120 \mathrm{mmol}, 84 \%$ ) as a clear colorless oil.

For TBS ether 6a:

$\mathrm{R}_{\mathrm{f}} 0.41$ (4:1 hexanes-EtOAc);

$[\alpha]_{\mathrm{D}}^{23}+68.4\left(c 0.95, \mathrm{CH}_{2} \mathrm{Cl}_{2}\right)$;

${ }^{1} \mathrm{H}-\mathrm{NMR}\left(400 \mathrm{MHz}, \mathrm{CDCl}_{3}\right) \delta$ 7.24-7.22 (m, 2H), 6.86-6.84 (m, 2H), $6.79(\mathrm{~d}, J=10.8$ $\mathrm{Hz}, 1 \mathrm{H}), 5.89(\mathrm{dd}, J=10.4 \mathrm{~Hz}, 1.6 \mathrm{~Hz}, 1 \mathrm{H}), 4.76\left(\mathrm{~A}\right.$ of $\left.\mathrm{AB}, J_{\mathrm{AB}}=6.6 \mathrm{~Hz}, 1 \mathrm{H}\right), 4.68(\mathrm{~B}$ of $\left.\mathrm{AB}, J_{\mathrm{AB}}=6.6 \mathrm{~Hz}, 1 \mathrm{H}\right), 4.55(\mathrm{~d}, J=9.6 \mathrm{~Hz}, 1 \mathrm{H}), 4.45\left(\mathrm{~A}\right.$ of $\left.\mathrm{AB}, J_{\mathrm{AB}}=11.2 \mathrm{~Hz}, 1 \mathrm{H}\right), 4.31$ $\left(\mathrm{B}\right.$ of $\left.\mathrm{AB}, J_{\mathrm{AB}}=11.2 \mathrm{~Hz}, 1 \mathrm{H}\right), 4.13(\mathrm{~d}, J=9.2 \mathrm{~Hz}, 1 \mathrm{H}), 3.80(\mathrm{~s}, 3 \mathrm{H}), 3.61(\mathrm{td}, J=11.2$ $\mathrm{Hz}, 4.0 \mathrm{~Hz}, 1 \mathrm{H}), 3.41(\mathrm{~s}, 3 \mathrm{H}), 3.35(\mathrm{~d}, J=9.2 \mathrm{~Hz}, 1 \mathrm{H}), 2.72(\mathrm{dt}, J=12.8,4.0 \mathrm{~Hz}, 1 \mathrm{H})$, $2.46(\mathrm{dd}, J=12.8,9.2 \mathrm{~Hz}, 1 \mathrm{H}), 2.06(\mathrm{td}, J=12.8,3.6 \mathrm{~Hz}, 1 \mathrm{H}), 1.86(\mathrm{~d}, J=11.2 \mathrm{~Hz}, 1 \mathrm{H})$, $1.61-1.54(\mathrm{~m}, 2 \mathrm{H}), 1.46-1.37(\mathrm{~m}, 2 \mathrm{H}), 1.34-1.11(\mathrm{~m}, 8 \mathrm{H}), 1.06(\mathrm{~d}, J=6.8 \mathrm{~Hz}, 3 \mathrm{H}), 0.96$ $(\mathrm{s}, 9 \mathrm{H}), 0.89$ (t, $J=6.8 \mathrm{~Hz}, 3 \mathrm{H}), 0.83(\mathrm{~s}, 3 \mathrm{H}), 0.19(\mathrm{~s}, 3 \mathrm{H}), 0.17(\mathrm{~s}, 3 \mathrm{H})$;

${ }^{13} \mathrm{C}-\mathrm{NMR}\left(100 \mathrm{MHz}, \mathrm{CDCl}_{3}\right) \delta 199.9,159.1,155.8,131.3,129.1,126.9,113.8,96.2$, 76.3, 73.5, 72.6, 56.4, 55.4, 50.2, 49.3, 44.3, 44.2, 32.8, 32.2, 31.4, 30.1, 29.9, 29.2, 26.2, $23.3,22.8,18.3,14.3,13.4,-3.1,-3.9$;

IR (film) 2954, 2926, 2855, 1682, 1514, 1463, 1384, 1362, 1249, 1171, 1075, 1043, 995, $854,835 \mathrm{~cm}^{-1}$;

HRMS (ES+) calc. for $\mathrm{C}_{36} \mathrm{H}_{60} \mathrm{O}_{6} \mathrm{SiNa}[\mathrm{M}+\mathrm{Na}]^{+}$639.4057. Found: 639.4076. 


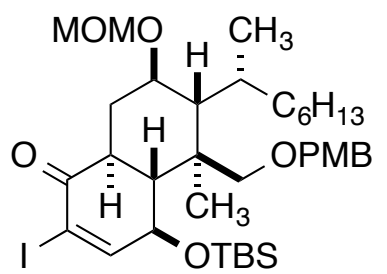

Compound 7

[Scheme 1, Step 4]

$\alpha$-Iodoketone 7: In a flame dried round-bottomed flask fitted with a magnetic stirbar and covered with aluminum foil was placed enone $\mathbf{6 a}(97.6 \mathrm{mg}, 0.158 \mathrm{mmol})$ dissolved in dry pyridine $(260 \mu \mathrm{L})$ and $\mathrm{CCl}_{4}(260 \mu \mathrm{L})$. To the solution was added $4-$

dimethylaminopyridine $(27.0 \mathrm{mg}, 0.22 \mathrm{mmol})$ and solid elemental iodine $(200.7 \mathrm{mg}$, $0.791 \mathrm{mmol}$ ) which was added portionwise over $2 \mathrm{~min}$. After a further $4 \mathrm{~h}$, a 1:1:1 solution of saturated $\mathrm{Na}_{2} \mathrm{~S}_{2} \mathrm{O}_{3}$ : saturated $\mathrm{NaHCO}_{3}: \mathrm{H}_{2} \mathrm{O}(15 \mathrm{~mL})$ was added and the mixture vigorously stirred for $15 \mathrm{~min}$. The mixture was then poured into ether $(20 \mathrm{~mL})$ and shaken. The layers were separated and the aqueous layer extracted with ether $(2 \times 10$ $\mathrm{mL})$. The combined organic portion was washed with dilute $\mathrm{HCl}(40 \mathrm{~mL}, 0.1 \mathrm{~N} \mathrm{HCl})$ and saturated $\mathrm{NaHCO}_{3}(2 \times 60 \mathrm{~mL})$ and then dried over anhydrous $\mathrm{Na}_{2} \mathrm{SO}_{4}$. The mixture was then filtered and concentrated in vacuo and the residue purified by flash chromatography (15 g SiO $2,15: 1-6: 1$ hexanes-EtOAc) afforded iodide 7 (110.3 mg, $0.1485 \mathrm{mmol}, 94 \%$ ) as a clear pale yellow oil.

For $\alpha$-iodo ketone 7:

$\mathrm{R}_{\mathrm{f}} 0.53$ (4:1 hexanes-EtOAc);

$[\alpha]_{D}^{22}+30.1\left(c 1.42, \mathrm{CH}_{2} \mathrm{Cl}_{2}\right)$

${ }^{1} \mathrm{H}-\mathrm{NMR}\left(400 \mathrm{MHz}, \mathrm{CDCl}_{3}\right) \delta 7.53(\mathrm{~d}, J=1.6 \mathrm{~Hz}, 1 \mathrm{H}), 7.24-7.21(\mathrm{~m}, 2 \mathrm{H}), 6.87-6.85$ $(\mathrm{m}, 2 \mathrm{H}), 4.75-4.68(\mathrm{~m}, 2 \mathrm{H}), 4.56(\mathrm{dd}, J=9.4,1.4 \mathrm{~Hz}, 1 \mathrm{H}), 4.44\left(\mathrm{~A}\right.$ of $\mathrm{AB}, J_{\mathrm{AB}}=11.2$ $\mathrm{Hz}, 1 \mathrm{H}), 4.30\left(\mathrm{~B}\right.$ of $\left.\mathrm{AB}, J_{\mathrm{AB}}=11.2 \mathrm{~Hz}, 1 \mathrm{H}\right), 4.06(\mathrm{~d}, J=9.2 \mathrm{~Hz}, 1 \mathrm{H}), 3.80(\mathrm{~s}, 3 \mathrm{H}), 3.60$ $(\mathrm{td}, J=10.8,4.0 \mathrm{~Hz}, 1 \mathrm{H}), 3.40(\mathrm{~s}, 3 \mathrm{H}), 3.33(\mathrm{~d}, J=9.2 \mathrm{~Hz}, 1 \mathrm{H}), 2.78(\mathrm{dt}, J=13.0,4.0$ $\mathrm{Hz}, 1 \mathrm{H}), 2.50(\mathrm{dd}, J=13.2,9.6, \mathrm{~Hz}, 1 \mathrm{H}), 2.20(\mathrm{td}, J=12.8 \mathrm{~Hz}, 4.0 \mathrm{~Hz}, 1 \mathrm{H}), 1.85(\mathrm{~d}, J=$ $11.2 \mathrm{~Hz}, 1 \mathrm{H}), 1.58-1.55(\mathrm{~m}, 1 \mathrm{H}), 1.41-1.08(\mathrm{~m}, 11 \mathrm{H}), 1.05(\mathrm{~d}, J=7.2 \mathrm{~Hz}, 3 \mathrm{H}), 0.97$ (s, $9 \mathrm{H}), 0.88(\mathrm{t}, J=6.8 \mathrm{~Hz}, 3 \mathrm{H}), 0.82(\mathrm{~s}, 3 \mathrm{H}), 0.21(\mathrm{~s}, 3 \mathrm{H}), 0.18(\mathrm{~s}, 3 \mathrm{H})$;

${ }^{13} \mathrm{C}-\mathrm{NMR}\left(100 \mathrm{MHz}, \mathrm{CDCl}_{3}\right) \delta 193.4,163.5,159.1,131.0,129.1,113.7,101.1,96.4$, 76.15, 74.1, 73.2, 72.6, 56.2, 55.4, 50.0, 49.2, 44.1, 42.9, 33.8, 32.7, 32.1, 31.3, 30.0, $29.1,26.1,23.2,22.8,18.3,14.2,13.4,-3.2,-4.0$;

IR (film) 2953, 2927, 2854, 1683, 1610, 1513, 1464, 1362, 1303, 1250, 1080, 1040, 831 $\mathrm{cm}^{-1}$;

HRMS (ES+) calc. for $\mathrm{C}_{36} \mathrm{H}_{59} \mathrm{IO}_{6} \mathrm{SiNa}[\mathrm{M}+\mathrm{Na}]^{+}$765.3023. Found: 765.3052. 


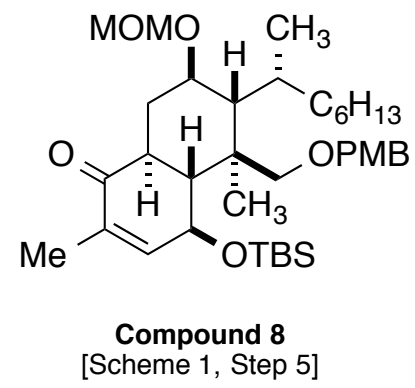

Enone 8: In a flame dried round-bottomed flask fitted with a magnetic stirbar was placed iodide $7(160.1 \mathrm{mg}, 0.216 \mathrm{mmol})$ dissolved in dry DMF $(2.1 \mathrm{~mL})$. The solution was then degassed briefly by stirring under high vacuum and backfilled with argon before $\mathrm{Pd}(\mathrm{dppf}) \mathrm{Cl}_{2} \cdot \mathrm{CH}_{2} \mathrm{Cl}_{2}$ ( $8.8 \mathrm{mg}, 0.0108 \mathrm{mmol}$ ), $\mathrm{CuI}(6.2 \mathrm{mg}, 0.0323 \mathrm{mmol})$, and $\mathrm{Me}_{4} \mathrm{Sn}$ $(208 \mu \mathrm{L}, 1.51 \mathrm{mmol})$ were added. The flask was then fitted with a reflux condenser and the reaction warmed to $60^{\circ} \mathrm{C}$. After $1 \mathrm{~h}$ the reaction was complete and the mixture was then poured into half saturated $\mathrm{NaCl}$ solution $(15 \mathrm{~mL})$. Ether was added and the layers separated and the aqueous layer extracted again with ether $(2 \times 15 \mathrm{~mL})$. The combined organic portion was washed with half saturated $\mathrm{NaCl}(20 \mathrm{~mL})$ and then dried over anhydrous $\mathrm{MgSO}_{4}$. The mixture was then filtered and concentrated in vacuo and the residue purified by flash chromatography $\left(12 \mathrm{~g} \mathrm{SiO}_{2}, 16: 1-6: 1\right.$ hexanes-EtOAc) affording enone 8 (127.1 $\mathrm{mg}, 0.2012 \mathrm{mmol}, 94 \%)$ as a clear colorless oil.

For enone 8:

$\mathrm{R}_{\mathrm{f}} 0.50$ (4:1 hexanes-EtOAc);

$[\alpha]_{\mathrm{D}}^{23}+48.0\left(c 0.65, \mathrm{CH}_{2} \mathrm{Cl}_{2}\right)$;

${ }^{1} \mathrm{H}-\mathrm{NMR}\left(400 \mathrm{MHz}, \mathrm{CDCl}_{3}\right) \delta$ 7.24-7.22 (m, 2H), 6.88-6.86 (m, 2H), $6.54(\mathrm{~s}, 1 \mathrm{H}), 4.76$ $\left(\mathrm{A}\right.$ of $\left.\mathrm{AB}, J_{\mathrm{AB}}=6.6 \mathrm{~Hz}, 1 \mathrm{H}\right), 4.69\left(\mathrm{~B}\right.$ of $\left.\mathrm{AB}, J_{\mathrm{AB}}=6.6 \mathrm{~Hz}, 1 \mathrm{H}\right), 4.50(\mathrm{td}, J=9.4,1.8 \mathrm{~Hz}$, $1 \mathrm{H}), 4.45\left(\mathrm{~A}\right.$ of $\left.\mathrm{AB}, J_{\mathrm{AB}}=11.3 \mathrm{~Hz}, 1 \mathrm{H}\right), 4.31\left(\mathrm{~B}\right.$ of $\left.\mathrm{AB}, J_{\mathrm{AB}}=11.3 \mathrm{~Hz}, 1 \mathrm{H}\right), 4.13(\mathrm{~d}, J=$ $9.2 \mathrm{~Hz}, 1 \mathrm{H}), 3.80(\mathrm{~s}, 3 \mathrm{H}), 3.61(\mathrm{td}, J=11.1,4.1 \mathrm{~Hz}, 1 \mathrm{H}), 3.41(\mathrm{~s}, 3 \mathrm{H}), 3.34(\mathrm{~d}, J=9.2$ $\mathrm{Hz}, 1 \mathrm{H}), 2.74(\mathrm{td}, J=13.1,3.9 \mathrm{~Hz}, 1 \mathrm{H}), 2.39(\mathrm{dd}, J=12.9,9.4 \mathrm{~Hz}, 1 \mathrm{H}), 2.02(\mathrm{td}, J=$ $12.5,3.7 \mathrm{~Hz}, 1 \mathrm{H}), 1.85(\mathrm{~d}, J=11.3 \mathrm{~Hz}, 1 \mathrm{H}), 1.74(\mathrm{~s}, 3 \mathrm{H}), 1.61-1.52(\mathrm{~m}, 1 \mathrm{H}), 1.48-1.38$ $(\mathrm{m}, 1 \mathrm{H}), 1.32-1.12(\mathrm{~m}, 10 \mathrm{H}), 1.06(\mathrm{~d}, J=6.8 \mathrm{~Hz}, 3 \mathrm{H}), 0.96(\mathrm{~s}, 9 \mathrm{H}), 0.88(\mathrm{t}, J=6.6 \mathrm{~Hz}$, $3 \mathrm{H}), 0.82(\mathrm{~s}, 3 \mathrm{H}), 0.19(\mathrm{~s}, 3 \mathrm{H}), 0.16(\mathrm{~s}, 3 \mathrm{H})$;

${ }^{13} \mathrm{C}-\mathrm{NMR}\left(100 \mathrm{MHz}, \mathrm{CDCl}_{3}\right) \delta 200.0,159.0,150.7,133.1,129.1,113.7,96.3,76.5,73.5$, $72.6,71.8,56.3,55.4,50.1,49.5,44.2,44.0,33.1,32.8,32.2,31.3,30.1,29.9,29.2,26.3$, $23.3,22.8,18.3,15.5,14.3,13.5,-3.1,-3.8$;

IR (film) 2955, 2927, 2855, 1676, 1613, 1513, 1465, 1364, 1301, 1249, 1080, 1044, 858, $836,777 \mathrm{~cm}^{-1}$;

HRMS (CI+) calc. for $\mathrm{C}_{37} \mathrm{H}_{61} \mathrm{O}_{6} \mathrm{Si}[\mathrm{M}-\mathrm{H}]^{+}$629.4232. Found: 629.4219. 


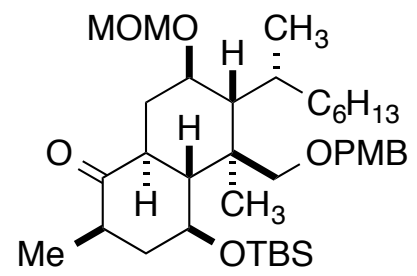

Compound 9

[Scheme 1, Step 6]

Ketone 9: In a flame dried round-bottomed flask fitted with a magnetic stirbar was placed enone $8(89.9 \mathrm{mg}, 0.142 \mathrm{mmol})$ dissolved in EtOAc $(7.2 \mathrm{~mL})$. $\mathrm{Pd} / \mathrm{C}(20 \mathrm{mg}, 5 \%$ $\mathrm{w} / \mathrm{w}, 140 \mathrm{mg} / \mathrm{mmol}$ enone) was added and the mixture degassed by applying vacuum and backfilling with argon before charging the flask with hydrogen using vacuum and backfilling from a ballon. The mixture was then stirred vigorously under a hydrogen filled balloon for $12 \mathrm{~h}$. The balloon was removed and the mixture purged of hydrogen by applying vacuum and back filling with argon several times. The mixture was then passed through a plug of silica $(2 \mathrm{~g})$ with EtOAc washing $(3 \times 5 \mathrm{~mL})$ and the filtrates concentrated in vacuo to afford ketone $9(87.7 \mathrm{mg}, 0.139 \mathrm{mmol}, 98 \%)$ as a clear colorless oil.

For ketone 9:

$\mathrm{R}_{\mathrm{f}} 0.50$ (4:1 hexanes-EtOAc);

$[\alpha]_{\mathrm{D}}^{22}+13.7\left(c 0.52, \mathrm{CH}_{2} \mathrm{Cl}_{2}\right)$;

${ }^{1} \mathrm{H}-\mathrm{NMR}\left(400 \mathrm{MHz}, \mathrm{CDCl}_{3}\right) \delta$ 7.24-7.22 (m, 2H), 6.86-6.84 (m, 2H), 4.72, (A of AB, $\left.J_{\mathrm{AB}}=6.8 \mathrm{~Hz}, 1 \mathrm{H}\right), 4.66\left(\mathrm{~B}\right.$ of $\left.\mathrm{AB}, J_{\mathrm{AB}}=6.8 \mathrm{~Hz}, 1 \mathrm{H}\right), 4.43\left(\mathrm{~A}\right.$ of $\left.\mathrm{AB}, J_{\mathrm{AB}}=11.6 \mathrm{~Hz}, 1 \mathrm{H}\right)$, $4.30\left(\mathrm{~B}\right.$ of $\left.\mathrm{AB}, J_{\mathrm{AB}}=11.6 \mathrm{~Hz}, 1 \mathrm{H}\right), 4.21(\mathrm{~d}, J=9.2 \mathrm{~Hz}, 1 \mathrm{H}), 4.11-4.04(\mathrm{~m}, 1 \mathrm{H}), 3.80(\mathrm{~s}$, $3 \mathrm{H}), 3.53(\mathrm{td}, J=11.2 \mathrm{~Hz}, 4.0 \mathrm{~Hz}, 1 \mathrm{H}), 3.39(\mathrm{~d}, J=9.2 \mathrm{~Hz}, 1 \mathrm{H}), 2.43-2.31(\mathrm{~m}, 2 \mathrm{H})$, $2.16-2.03(\mathrm{~m}, 3 \mathrm{H}), 1.86(\mathrm{~d}, J=11.6 \mathrm{~Hz}, 1 \mathrm{H}), 1.58-1.46(\mathrm{~m}, 2 \mathrm{H}), 1.45-1.20(\mathrm{~m}, 11 \mathrm{H})$, $1.04(\mathrm{~d}, J=6.8 \mathrm{~Hz}, 3 \mathrm{H}), 1.01(\mathrm{~d}, J=6.6 \mathrm{~Hz}, 3 \mathrm{H}), 0.92-0.82(\mathrm{~m}, 15 \mathrm{H}), 0.14(\mathrm{~s}, 3 \mathrm{H}), 0.13$ $(\mathrm{s}, 3 \mathrm{H})$

${ }^{13} \mathrm{C}-\mathrm{NMR}\left(100 \mathrm{MHz}, \mathrm{CDCl}_{3}\right) \delta 211.5,159.0,131.4,129.0,113.7,96.3,76.8,73.1,73.0$, $72.5,56.3,55.4,50.3,49.9,45.4,44.7,44.5,42.1,32.8,32.5,32.2,31.5,30.1,29.1,26.3$, $23.2,22.8,18.3,14.6,14.3,12.7,-3.4,-3.7$;

IR (film) 2954, 2926, 2854, 1718, 1513, 1462, 1248, 1083, 1039, 852, $843 \mathrm{~cm}^{-1}$;

HRMS (ES+) calc. for $\mathrm{C}_{37} \mathrm{H}_{64} \mathrm{O}_{6} \mathrm{SiNa}[\mathrm{M}+\mathrm{Na}]^{+}$655.4370. Found: 655.4379 . 


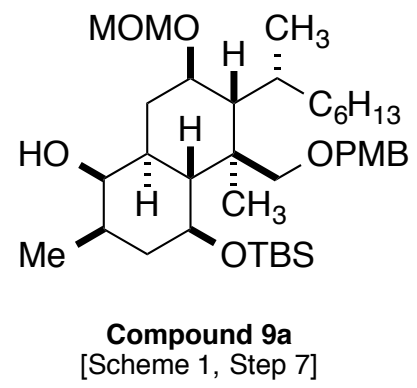

Alcohol 9a: To a $50 \mathrm{~mL}$ round-bottom flask containing ketone 9 (218 $\mathrm{mg}, 0.344 \mathrm{mmol})$ under argon was added THF $(11.1 \mathrm{~mL}, 0.03 \mathrm{M})$. The reaction mixture was cooled to -78 ${ }^{\circ} \mathrm{C}$, followed by the addition of L-selectride ${ }^{\circledR}(1.38 \mathrm{~mL}, 1.38 \mathrm{mmol}, 1 \mathrm{M}$ in THF). The solution was stirred at $-78{ }^{\circ} \mathrm{C}$ until no starting material remained by TLC, approximately $2.5 \mathrm{~h}$. At which time a solution of $\mathrm{NaOH}(7.5 \mathrm{~mL}, 1.0 \mathrm{M})$ and $30 \% \mathrm{H}_{2} \mathrm{O}_{2}(6.0 \mathrm{~mL})$ was added to the reaction mixture at $-78{ }^{\circ} \mathrm{C}$ and warmed to $0{ }^{\circ} \mathrm{C}$ and stirred for $1 \mathrm{~h}$. The solution was diluted with $\mathrm{H}_{2} \mathrm{O}(15 \mathrm{~mL})$ and EtOAc $(50 \mathrm{~mL})$. The layers were then separated and the aqueous layer was extracted with EtOAc $(2 \times 50 \mathrm{~mL})$. The combined organic layers were washed with $\mathrm{H}_{2} \mathrm{O}(1 \times 50 \mathrm{~mL})$, saturated $\mathrm{NH}_{4} \mathrm{Cl}(1 \times 50 \mathrm{~mL})$, dried over anhydrous $\mathrm{Na}_{2} \mathrm{SO}_{4}$, filtered and concentrated in vacuo. The residue was then purified by flash column chromatography (95:5-3:1 hexanes-EtOAc) to afford 9a (195 $\mathrm{mg}, 0.307 \mathrm{mmol}, 90 \%$ ) as a colorless oil.

For alcohol 9a:

$\mathrm{R}_{\mathrm{f}} 0.29$ (4:1 hexanes-EtOAc);

$[\alpha]_{\mathrm{D}}^{20}+23.7\left(c 0.35, \mathrm{CH}_{2} \mathrm{Cl}_{2}\right)$;

${ }^{1} \mathrm{H}-\mathrm{NMR}\left(500 \mathrm{MHz}, \mathrm{CDCl}_{3}\right) \delta 7.26-7.25(\mathrm{~m}, 2 \mathrm{H}), 6.87-6.85(\mathrm{~m}, 2 \mathrm{H}), 4.70$ (A of AB, $\left.J_{\mathrm{AB}}=6.8 \mathrm{~Hz}, 1 \mathrm{H}\right), 4.68\left(\mathrm{~B}\right.$ of AB, $\left.J_{\mathrm{AB}}=6.8 \mathrm{~Hz}, 1 \mathrm{H}\right), 4.47\left(\mathrm{~A}\right.$ of AB, $\left.J_{\mathrm{AB}}=11.5 \mathrm{~Hz}, 1 \mathrm{H}\right)$, $4.31\left(\mathrm{~B}\right.$ of $\left.\mathrm{AB}, J_{\mathrm{AB}}=11.5 \mathrm{~Hz}, 1 \mathrm{H}\right), 4.22(\mathrm{~d}, J=7.2 \mathrm{~Hz}, 1 \mathrm{H}), 3.80(\mathrm{~s}, 3 \mathrm{H}), 3.62-3.55(\mathrm{~m}$, $2 \mathrm{H}), 3.41-3.36(\mathrm{~m}, 4 \mathrm{H}), 3.29$ (d, $J=9.0 \mathrm{~Hz}, 1 \mathrm{H}), 2.02-1.96(\mathrm{~m}, 2 \mathrm{H}), 1.88(\mathrm{~d}, J=11.0$ $\mathrm{Hz}, 1 \mathrm{H}), 1.60-1.08(\mathrm{~m}, 17 \mathrm{H}), 1.03(\mathrm{~d}, J=7.0 \mathrm{~Hz}, 3 \mathrm{H}), 0.96(\mathrm{~m}, 3 \mathrm{H}), 0.92-0.84(\mathrm{~m}$, $15 \mathrm{H}), 0.73(\mathrm{~s}, 3 \mathrm{H}), 0.10(\mathrm{~s}, 3 \mathrm{H}), 0.08(\mathrm{~s}, 3 \mathrm{H})$;

${ }^{13} \mathrm{C}-\mathrm{NMR}\left(100 \mathrm{MHz}, \mathrm{CDCl}_{3}\right) \delta 158.9,131.8,129.1,113.7,96.5,77.7,75.2,74.7,74.3$, 72.6, 56.1, 55.4, 50.8, 43.5, 41.9, 39.7, 39.6, 37.3, 35.1, 32.9, 32.2, 31.5, 30.2, 29.1, 26.4, $23.2,22.8,18.4,17.9,14.3,13.1,-3.3,-3.7$;

IR (film) 3446 (br), 2958, 2923, 2851, 1613, 1514, 1463, 1377, 1249, 1083, 1042, 834 $\mathrm{cm}^{-1}$;

HRMS (ES+) calc. for $\mathrm{C}_{37} \mathrm{H}_{66} \mathrm{O}_{6} \mathrm{SiNa}[\mathrm{M}+\mathrm{Na}]^{+}$657.4526. Found: 657.4528. 


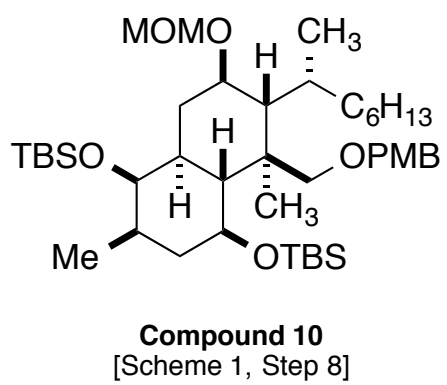

Bis-TBS Ether 10: To a $50 \mathrm{~mL}$ round-bottom flask containing alcohol 9a (256 mg, 0.403 mmol) under argon was added $\mathrm{CH}_{2} \mathrm{Cl}_{2}(8.1 \mathrm{~mL}, 0.016 \mathrm{M})$ and 2,6-di-tert-butylpyridine $(0.712 \mathrm{~mL}, 3.22 \mathrm{mmol})$. The reaction mixture was cooled to $-40{ }^{\circ} \mathrm{C}$, followed by the addition of TBSOTf $(0.612 \mathrm{~mL}, 0.575 \mathrm{mmol})$. The solution was allowed to slowly warm to $-10{ }^{\circ} \mathrm{C}$ over $30 \mathrm{~min}$ and then recooled to $-40{ }^{\circ} \mathrm{C}$. The reaction mixture was quenched by the addition of saturated $\mathrm{NaHCO}_{3}(15 \mathrm{~mL})$ and the cooling bath removed. After 20 min the solution was diluted with EtOAc $(50 \mathrm{~mL})$ and $\mathrm{H}_{2} \mathrm{O}(15 \mathrm{~mL})$. The layers were then separated and the aqueous layer was extracted with EtOAc $(2 \times 50 \mathrm{~mL})$. The combined organic layers were dried over anhydrous $\mathrm{Na}_{2} \mathrm{SO}_{4}$, filtered and concentrated in vacuo. Purification by flash chromatography (98:2-95:5 hexanes-ethyl acetate) provided 10 (309 mg, $0.395 \mathrm{mmol}, 98 \%$ ) as a colorless oil.

For TBS ether 10:

$\mathrm{R}_{\mathrm{f}} 0.54$ (6:1 hexanes-EtOAc);

$[\alpha]_{\mathrm{D}}^{23}+37.0\left(c 0.35 \mathrm{CH}_{2} \mathrm{Cl}_{2}\right)$

${ }^{1} \mathrm{H}-\mathrm{NMR}\left(400 \mathrm{MHz}, \mathrm{CDCl}_{3}\right) \delta$ 7.26-7.23 (m, 2H), 6.85-6.83 (m, 2H), $4.68(\mathrm{~s}, 2 \mathrm{H}), 4.45$ $\left(\mathrm{A}\right.$ of $\left.\mathrm{AB}, J_{\mathrm{AB}}=11.6 \mathrm{~Hz}\right), 4.31(\mathrm{~m}, 2 \mathrm{H}), 3.80(\mathrm{~s}, 3 \mathrm{H}), 3.58(\mathrm{td}, J=10.0,4.0 \mathrm{~Hz}, 1 \mathrm{H}), 3.50$ (td, $J=11.2,4.0 \mathrm{~Hz}, 1 \mathrm{H}), 3.43(\mathrm{br} \mathrm{s}, 1 \mathrm{H}), 3.37$ (s, 3H), $3.28(\mathrm{~d}, J=9.2 \mathrm{~Hz}, 1 \mathrm{H}), 2.18$ (dd, $J=11.6,10.0 \mathrm{~Hz}, 1 \mathrm{H}), 1.90-1.85(\mathrm{~m}, 2 \mathrm{H}), 1.68-1.14(\mathrm{~m}, 16 \mathrm{H}), 1.03(\mathrm{~d}, J=6.8 \mathrm{~Hz}, 3 \mathrm{H})$, 0.91-0.85 (m, 24H), 0.73 (s, 3H), 0.09 (s, 3H), 0.08 (s, 3H), 0.07 (s, 3H), 0.03 (s, 3H);

${ }^{13} \mathrm{C}-\mathrm{NMR}\left(100 \mathrm{MHz}, \mathrm{CDCl}_{3}\right) \delta 158.6,132.3,128.6,113.6,96.9,78.7,76.3,75.3,74.3$, 72.3, 56.0, 55.4, 50.7, 43.6, 41.6, 41.0, 40.0, 39, 36.4, 33.0, 32.2, 31.6, 30.2, 29.2, 26.5, $23.3,22.9,19.3,18.7,18.4,14.3,13.0,-2.9,-3.1,-3.4,-3.4$;

IR (film) 2954, 2928, 2855, 1617, 1514, 1463, 1361, 1249, 1172, 1147, 1099, 1045, $1000,921,935,773 \mathrm{~cm}^{-1}$;

HRMS (ES+) calc. for $\mathrm{C}_{43} \mathrm{H}_{80} \mathrm{O}_{6} \mathrm{Si}_{2} \mathrm{Na}[\mathrm{M}+\mathrm{Na}]^{+}$771.5391. Found: 771.5414. 


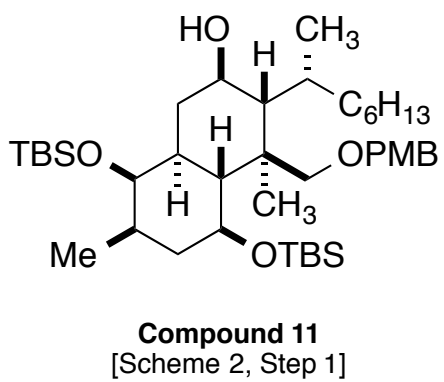

Alcohol 11:To a $50 \mathrm{~mL}$ round-bottom flask containing MOM ether 10 (150 mg, 0.192 $\mathrm{mmol})$ under argon was added $\mathrm{CH}_{2} \mathrm{Cl}_{2}(12.0 \mathrm{~mL}, 0.016 \mathrm{M})$ and 2,6-di-tert-butylpyridine $(0.424 \mathrm{~mL}, 1.92 \mathrm{mmol})$. The reaction mixture was cooled to $-78^{\circ} \mathrm{C}$, followed by the addition of $B$-bromocatecholborane $\left(0.612 \mathrm{~mL}, 0.575 \mathrm{mmol}, 0.94 \mathrm{M}\right.$ in $\left.\mathrm{CH}_{2} \mathrm{Cl}_{2}\right)$. The solution was stirred at $-78^{\circ} \mathrm{C}$ until no starting material remained by TLC, at which time the mixture was poured into a 1:1 mixture of THF/pH 7 buffer $(100 \mathrm{~mL})$ and agitated briefly. $\mathrm{Et}_{2} \mathrm{O}(100 \mathrm{~mL})$ was added and the layers were separated. The aqueous layer was extracted with $\mathrm{Et}_{2} \mathrm{O}(100 \mathrm{~mL})$ and the combined organic layers washed with saturated $\mathrm{NaHCO}_{3}(75 \mathrm{~mL})$, dried over anhydrous $\mathrm{Na}_{2} \mathrm{SO}_{4}$, filtered and concentrated in vacuo. The residue was then purified by flash column chromatography (98:2-6:1 hexanes-EtOAc) to afford alcohol $11(127 \mathrm{mg}, 0.180 \mathrm{mmol}, 94 \%)$ as a colorless oil.

For alcohol 11:

$\mathrm{R}_{\mathrm{f}} 0.48$ (6:1 hexanes-EtOAc);

$[\alpha]_{D}^{20}+21.0\left(c 0.14, \mathrm{CH}_{2} \mathrm{Cl}_{2}\right)$;

${ }^{1} \mathrm{H}-\mathrm{NMR}\left(400 \mathrm{MHz}, \mathrm{CDCl}_{3}\right) \delta 7.25-7.23(\mathrm{~m}, 2 \mathrm{H}), 6.85-6.83(\mathrm{~m}, 2 \mathrm{H}), 4.43$ (A of AB, $\left.J_{\mathrm{AB}}=11.6 \mathrm{~Hz}, 1 \mathrm{H}\right), 4.33-4.29(\mathrm{~m}, 2 \mathrm{H}), 3.80(\mathrm{~s}, 3 \mathrm{H}), 3.69(\mathrm{~m}, 1 \mathrm{H}), 3.58(\mathrm{td}, J=10.0,4.0$ $\mathrm{Hz}, 1 \mathrm{H}), 3.43$ (br s, 1H), 3.27 (d, $J=9.2 \mathrm{~Hz}, 1 \mathrm{H}), 2.17$ (dd, $J=11.6,9.8 \mathrm{~Hz}, 1 \mathrm{H}), 1.69$ $1.05(\mathrm{~m}, 19 \mathrm{H}), 1.05(\mathrm{~d}, J=6.8 \mathrm{~Hz}, 3 \mathrm{H}), 0.90-0.85(\mathrm{~m}, 24 \mathrm{H}), 0.73(\mathrm{~s}, 3 \mathrm{H}), 0.08(\mathrm{~s}, 3 \mathrm{H})$, $0.07(\mathrm{~s}, 3 \mathrm{H}), 0.05(\mathrm{~s}, 3 \mathrm{H}), 0.03(\mathrm{~s}, 3 \mathrm{H})$;

${ }^{13} \mathrm{C}-\mathrm{NMR}\left(100 \mathrm{MHz}, \mathrm{CDCl}_{3}\right) \delta 158.7,132.3,128.6,113.6,94.6,76.1,75.3,74.2,72.3$, 69.9, 55.4, 53.0, 43.7, 42.0, 41.6, 41.3, 40.1, 36.4, 33.1, 30.1, 29.1, 26.5, 23.9, 22.9, 19.3, $18.7,18.4,14.3,13.0,-2.9,-3.0,-3.4$

IR (film) 3483 (br), 2954, 2927, 2855, 1614, 1513, 1463, 1378, 1301, 1248, 1171, 1140, 1064, 1040, 927, 882, 834, $774 \mathrm{~cm}^{-1}$;

HRMS (CI+) calc. for $\mathrm{C}_{41} \mathrm{H}_{77} \mathrm{O}_{5} \mathrm{Si}_{2}[\mathrm{M}+\mathrm{H}]^{+}$705.5304. Found: 705.5326. 


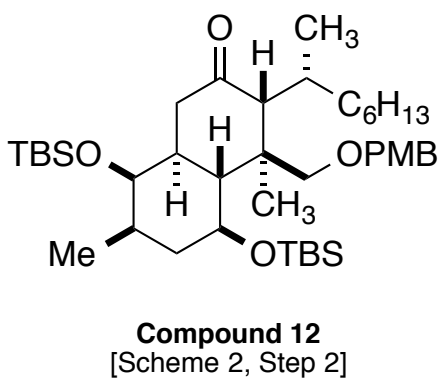

Ketone 12: In a flame dried round-bottomed flask fitted with a magnetic stirbar was placed alcohol $11(5.1 \mathrm{mg}, 0.0071 \mathrm{mmol})$ dissolved in dry $\mathrm{CH}_{2} \mathrm{Cl}_{2}(150 \mu \mathrm{L})$. To this was added powdered $4 \AA$ activated molecular sieves and the mixture was allowed to stir for 5 min. $N$-methylmorpholine- $N$-oxide $(1.3 \mathrm{mg}, 0.011 \mathrm{mmol})$ and tetra- $N$-propylammonium perruthenate $(0.2 \mathrm{mg}, 0.0006 \mathrm{mmol})$ were added. The mixture was allowed to stir for 30 min before the the reaction mixture was taken up and filtered through a plug of Celite ${ }^{\circledR}$ and concentrated in vacuo. The residue was then purified by flash column chromatography (1.5 $\mathrm{g} \mathrm{SiO}_{2}, 50: 1-20: 1$ hexanes-EtOAc) to afford ketone 12 (4.2 $\mathrm{mg}$, $0.0058 \mathrm{mmol}, 80 \%$ ) as a clear colorless oil.

For ketone 12:

$\mathrm{R}_{\mathrm{f}} 0.52$ (6:1 hexanes-EtOAc);

$[\alpha]_{\mathrm{D}}^{21}+24.1\left(c 0.29, \mathrm{CH}_{2} \mathrm{Cl}_{2}\right)$;

${ }^{1} \mathrm{H}-\mathrm{NMR}\left(400 \mathrm{MHz}, \mathrm{CDCl}_{3}\right) \delta 7.24-7.22(\mathrm{~m}, 2 \mathrm{H}), 6.86-6.84(\mathrm{~m}, 2 \mathrm{H}), 4.51$ (A of AB, $J=$ $11.6 \mathrm{~Hz}, 1 \mathrm{H}), 4.39(\mathrm{~d}, J=8.8 \mathrm{~Hz}, 1 \mathrm{H}), 4.30(\mathrm{~B}$ of AB, $J=11.6 \mathrm{~Hz}, 1 \mathrm{H}), 3.81(\mathrm{~s}, 3 \mathrm{H})$, $3.66(\mathrm{td}, J=10.0,4.0 \mathrm{~Hz}, 1 \mathrm{H}), 3.39$ (br s, $1 \mathrm{H}), 3.19$ (d, $J=8.8 \mathrm{~Hz}, 1 \mathrm{H}), 2.86($ br s, $1 \mathrm{H})$, 2.60-2.50 (m, 2H), $1.95(\mathrm{dd}, J=12.4,4.8 \mathrm{~Hz}, 1 \mathrm{H}), 1.72-1.05(\mathrm{~m}, 15 \mathrm{H}), 0.93-0.85(\mathrm{~m}$, $30 \mathrm{H}), 0.11(\mathrm{~s}, 3 \mathrm{H}), 0.10(\mathrm{~s}, 3 \mathrm{H}), 0.05(\mathrm{~s}, 3 \mathrm{H}), 0.01(\mathrm{~s}, 3 \mathrm{H})$;

${ }^{13} \mathrm{C}-\mathrm{NMR}\left(100 \mathrm{MHz}, \mathrm{CDCl}_{3}\right) \delta 214.8,209.0,170.3,131.8,128.7,113.6,75.4,74.7,74.3$, $72.2,59.8,55.4,48.3,47.4,44.5,43.2,40.1,35.9,34.1,32.2,30.8,29.5,26.5,26.4,22.9$, $20.8,19.3,18.8,18.5,14.7,14.3,-2.7,-3.1,-3.5,-4.8$;

IR (film) 2955, 2923, 2856, 1715, 1616, 1513, 1463, 1380, 1249, 1170, 1063, 1040, $1004,884,835,774 \mathrm{~cm}^{-1}$;

HRMS (ES+) calc. for $\mathrm{C}_{41} \mathrm{H}_{74} \mathrm{O}_{5} \mathrm{Si}_{2} \mathrm{Na}[\mathrm{M}+\mathrm{Na}]^{+}$725.4973. Found: 725.4993 . 


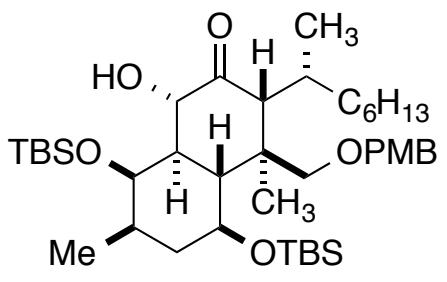

Compound 13

[Scheme 2, Step 3]

Ketol 13: A flame-dried $25 \mathrm{~mL}$ round-bottom flask charged with a magnetic stir bar was evacuated and back-filled with argon. A THF $(3.0 \mathrm{~mL})$ solution of ketone $12(228 \mathrm{mg}$, $0.324 \mathrm{mmol}$ ) was slowly added down the sides of the flask to a lithium diisopropylamide (3.89 mL, 3.89 mmol, 1.0 M in THF) solution at $-78^{\circ} \mathrm{C}$. Additional THF (3.5 mL) was used to rinse the remaining ketone into the reaction flask. The solution was allowed to stir at $-78^{\circ} \mathrm{C}$ for $2 \mathrm{~h}$ before freshly distilled TMSCl $(0.62 \mathrm{~mL}, 4.86 \mathrm{mmol})$ was added dropwise via syringe over $5 \mathrm{~min}$. The mixture was allowed to stir for $3 \mathrm{~h}$ at $-78{ }^{\circ} \mathrm{C}$. A solution of pH 7 buffer $(75 \mathrm{~mL})$ was cooled in an Erlenmeyer flask to $0{ }^{\circ} \mathrm{C}$ and then the silyl enol ether solution was poured into the cooled buffer solution and agitated briefly. Pentane $(150 \mathrm{~mL})$ was added to the quenched reaction mixture and the layers were separated. The aqueous layer was extracted with pentane $(2 \times 100 \mathrm{~mL})$ and the combined organic layers were dried over anhydrous $\mathrm{Na}_{2} \mathrm{SO}_{4}$, filtered and concentrated to provide a yellow oil. The crude product was used in the following reaction without purification. To a $50 \mathrm{~mL}$ round-bottom flask containing the crude silyl enol ether was added $\mathrm{CH}_{2} \mathrm{Cl}_{2}$ (15.4 $\mathrm{mL}, 0.021 \mathrm{M}$ ). The reaction mixture was cooled to $0{ }^{\circ} \mathrm{C}$, followed by addition of anhydrous $\mathrm{NaHCO}_{3}(544 \mathrm{mg}, 6.48 \mathrm{mmol})$ and $\mathrm{m}$-chloroperoxybenzoic acid (227 mg, $1.30 \mathrm{mmol}$ ). The reaction was stirred at $0{ }^{\circ} \mathrm{C}$ for $1 \mathrm{~h}$, at which time a $10 \%$ solution of $\mathrm{Na}_{2} \mathrm{SO}_{3}(25 \mathrm{~mL})$ was added and the solution was stirred for an additional 15 min while warming to room temperature. The solution was diluted with $\mathrm{H}_{2} \mathrm{O}(15 \mathrm{~mL})$ and $\mathrm{CH}_{2} \mathrm{Cl}_{2}$ $(75 \mathrm{~mL})$. The layers were then separated and the aqueous layer was extracted with $\mathrm{CH}_{2} \mathrm{Cl}_{2}(2 \times 100 \mathrm{~mL})$. The combined organic layers were washed with saturated $\mathrm{NaHCO} 3(1 \times 50 \mathrm{~mL})$ and saturated $\mathrm{NaCl}(1 \times 50 \mathrm{~mL})$. The organic layer was then dried over anhydrous $\mathrm{Na}_{2} \mathrm{SO}_{4}$, filtered and concentrated to provide a yellow oil. The crude product was used in the following reaction without purification. To a $50 \mathrm{~mL}$ roundbottom flask containing the crude TMS ether was added $\mathrm{CH}_{2} \mathrm{Cl}_{2}(16.2 \mathrm{~mL}, 0.02 \mathrm{M})$ and $\mathrm{MeOH}(0.77 \mathrm{~mL}, 0.42 \mathrm{M})$. The reaction mixture was cooled to $0{ }^{\circ} \mathrm{C}$, followed by addition of camphorsulfonic acid $(7.5 \mathrm{mg}, 0.0324 \mathrm{mmol})$. The reaction was stirred at $0{ }^{\circ} \mathrm{C}$ until no starting material remained by TLC, approximately $1 \mathrm{~h} 15 \mathrm{~min}$. A saturated solution of $\mathrm{NaHCO}_{3}(20 \mathrm{~mL})$ was added to the reaction at $0{ }^{\circ} \mathrm{C}$. The solution was diluted with $\mathrm{H}_{2} \mathrm{O}(20 \mathrm{~mL})$ and $\mathrm{CH}_{2} \mathrm{Cl}_{2}(75 \mathrm{~mL})$. The layers were then separated and the aqueous layer was extracted with $\mathrm{CH}_{2} \mathrm{Cl}_{2}(2 \times 100 \mathrm{~mL})$. The combined organic layers were dried over anhydrous $\mathrm{Na}_{2} \mathrm{SO}_{4}$, filtered and concentrated in vacuo. Purification by flash chromatography (95:5-9:1 hexanes-ethyl acetate) provided ketol 13 (201 mg, 0.279 mmol, $86 \%$ over 2 steps) as a colorless oil.

For ketol 13: 
$\mathrm{R}_{\mathrm{f}} 0.62(6: 1$ hexanes-EtOAc);

$[\alpha]_{23}^{\mathrm{D}}+12.0\left(\mathrm{c} 1.20, \mathrm{CH}_{2} \mathrm{Cl}_{2}\right)$;

${ }^{1} \mathrm{H}-\mathrm{NMR}\left(400 \mathrm{MHz}, \mathrm{CDCl}_{3}\right) \delta$ 7.28-7.19 (m, 2H), 6.88-6.82 (m, 2H), 4.52 (A of AB, $\left.J_{\mathrm{AB}}=11.4 \mathrm{~Hz}, 1 \mathrm{H}\right), 4.46(\mathrm{~d}, J=8.8,1 \mathrm{H}), 4.30\left(\mathrm{~B}\right.$ of AB, $\left.J_{\mathrm{AB}}=11.3 \mathrm{~Hz}, 1 \mathrm{H}\right), 3.97$ (bs, 1H) $3.93(\mathrm{dd}, J=10.5,3.4 \mathrm{~Hz}, 1 \mathrm{H}), 3.81(\mathrm{~s}, 3 \mathrm{H}), 3.62$ (ddd, $J=10.4,10.4,3.8 \mathrm{~Hz}, 1 \mathrm{H})$, $3.60(\mathrm{~d}, J=3.7 \mathrm{~Hz}, 1 \mathrm{H}), 3.19(\mathrm{~d}, J=8.8 \mathrm{~Hz}, 1 \mathrm{H}), 2.86(\mathrm{bs}, 1 \mathrm{H}), 2.69(\mathrm{dd}, J=11.7,9.7$ $\mathrm{Hz}, 1 \mathrm{H}), 1.83-0.77(\mathrm{~m}, 24 \mathrm{H}), 0.92(\mathrm{~s}, 9 \mathrm{H}), 0.91(\mathrm{~s}, 9 \mathrm{H}), 0.84(\mathrm{~s}, 3 \mathrm{H}), 0.11(\mathrm{~s}, 3 \mathrm{H}), 0.10$ (s, 3H), 0.10 (s, 3H), 0.09 (s, 3H);

${ }^{13} \mathrm{C}-\mathrm{NMR}\left(100 \mathrm{MHz}, \mathrm{CDCl}_{3}\right) \delta 214.6,158.9,131.5,128.7,113.7,74.8,73.9,73.7,72.2$, $71.1,57.3,55.4,54.0,49.4,41.7,40.1,35.7,33.9,32.2,30.6,29.8,29.4,26.5,26.4,22.8$, $20.4,19.2,18.8,18.4,14.5,14.3,-2.9,-3.1,-3.7,-4.4$;

IR (film) 3468, 2955, 2928, 2856, 1712, 1721, 1613, 1514, 1463, 1385, 1251, 1065, $1040,988,836,775 \mathrm{~cm}^{-1}$;

HRMS (ESI+) calc. for $\mathrm{C}_{41} \mathrm{H}_{74} \mathrm{O}_{6} \mathrm{Si}_{2} \mathrm{Na}[\mathrm{M}+\mathrm{Na}]^{+}$741.4916. Found 741.4955. 


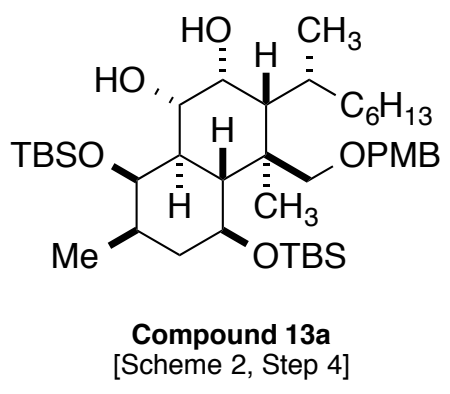

Diol 13a: To a $100 \mathrm{~mL}$ round-bottom flask containing 13 under argon was added $\mathrm{MeOH}$ (36.1 mL, $0.02 \mathrm{M})$. The solution was cooled to $0{ }^{\circ} \mathrm{C}$ and $\mathrm{NaBH}_{4}(682 \mathrm{mg}, 18.0 \mathrm{mmol})$ was added in two equal portions to the reaction. The reaction mixture was stirred at $0{ }^{\circ} \mathrm{C}$ until no starting material was present by TLC, approximately $1 \mathrm{~h}$. A saturated solution of $\mathrm{NH}_{4} \mathrm{Cl}(30 \mathrm{~mL})$ was added slowly to the reaction at $0{ }^{\circ} \mathrm{C}$, followed by stirring for $15 \mathrm{~min}$ while warming to room temperature. The solution was diluted with $\mathrm{H}_{2} \mathrm{O}(15 \mathrm{~mL})$ and EtOAc $(75 \mathrm{~mL})$. The layers were then separated and the aqueous layer was extracted with EtOAc $(2 \times 100 \mathrm{~mL})$. The combined organic layers were washed with saturated $\mathrm{NaCl}(1 \mathrm{x}$ $50 \mathrm{~mL}$ ), dried over anhydrous $\mathrm{Na}_{2} \mathrm{SO}_{4}$, filtered and concentrated in vacuo. Purification by flash chromatography (98:2-9:1 hexanes-ethyl acetate) provided diol 13a (460 mg, 0.638 mmol, $88 \%$ ) as a viscous colorless oil.

\section{For diol 13a:}

$\mathrm{R}_{\mathrm{f}} 0.34$ (6:1 hexanes-EtOAc);

$[\alpha] \mathrm{D} 23+32.7\left(\mathrm{c} 0.80, \mathrm{CH}_{2} \mathrm{Cl}_{2}\right)$;

${ }^{1} \mathrm{H}-\mathrm{NMR}\left(400 \mathrm{MHz}, \mathrm{CDCl}_{3}\right) \delta$ 7.23-7.17 (m, 2H), 6.86-6.81 (m, 2H), 4.43 (A of AB, $\left.J_{\mathrm{AB}}=11.7 \mathrm{~Hz}, 1 \mathrm{H}\right), 4.28\left(\mathrm{~B}\right.$ of $\left.\mathrm{AB}, J_{\mathrm{AB}}=11.7 \mathrm{~Hz}, 1 \mathrm{H}\right), 4.27(\mathrm{~d}, J=9.0 \mathrm{~Hz}, 1 \mathrm{H}), 4.12-$ $4.09(\mathrm{~m}, 1 \mathrm{H}), 4.04(\mathrm{bs}, 1 \mathrm{H}), 3.80(\mathrm{~s}, 3 \mathrm{H}), 3.70(\mathrm{ddd}, J=10.5,10.5,4.1 \mathrm{~Hz}, 1 \mathrm{H}), 3.36$ (ddd, $J=11.0,8.3,2.8 \mathrm{~Hz}, 1 \mathrm{H}), 3.20(\mathrm{~d}, J=8.9 \mathrm{~Hz}, 1 \mathrm{H}), 2.29(\mathrm{dd}, J=11.6,9.8 \mathrm{~Hz}, 1 \mathrm{H})$, 1.95-0.81 (m, 26H), $1.84(\mathrm{~d}, J=8.3 \mathrm{~Hz}, 1 \mathrm{H}), 1.01(\mathrm{~s}, 3 \mathrm{H}), 0.89(\mathrm{~s}, 9 \mathrm{H}), 0.88(\mathrm{~s}, 9 \mathrm{H})$, $0.10(\mathrm{~s}, 3 \mathrm{H}), 0.09(\mathrm{~s}, 3 \mathrm{H}), 0.07(\mathrm{~s}, 3 \mathrm{H}), 0.06(\mathrm{~s}, 3 \mathrm{H})$;

${ }^{13} \mathrm{C}-\mathrm{NMR}\left(100 \mathrm{MHz}, \mathrm{CDCl}_{3}\right) \delta 158.8,132.1,128.8,113.6,75.3,74.8,72.4,71.4,71.2$, $70.3,55.5,47.0,44.5,41.5,40.9,40.3,36.2,35.2,32.3,30.5,30.0,29.4,26.6,26.5,22.9$, $21.5,19.5,18.8,18.5,15.7,14.3,-3.0,-3.2,-3.5,-4.0$;

IR (film) 3445, 2955, 2928, 2856, 1614, 1514, 1463, 1386, 1301, 1249, 1171, 1089, $1063,1037,984,940,911,831,774,674 \mathrm{~cm}^{-1}$;

HRMS (ESI+) calc. for $\mathrm{C}_{41} \mathrm{H}_{76} \mathrm{O}_{6} \mathrm{Si}_{2} \mathrm{Na}[\mathrm{M}+\mathrm{Na}]^{+}$743.5073. Found 743.5093. 


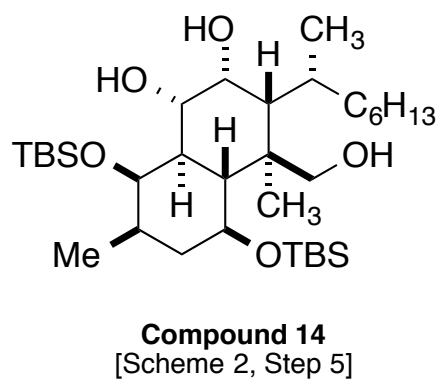

Triol 14: To a $100 \mathrm{~mL}$ round-bottom flask containing diol 13a was added a 1:1 solution of $\mathrm{CH}_{2} \mathrm{Cl}_{2}: \mathrm{pH} 7$ buffer $(31.9 \mathrm{~mL}, 0.02 \mathrm{M})$. The solution was cooled to $0{ }^{\circ} \mathrm{C}$, DDQ (434 $\mathrm{mg}, 1.91 \mathrm{mmol}$ ) was added to the reaction and the mixture was allowed to warm to room temperature. After stirring for $5 \mathrm{~h}$, a saturated solution of $\mathrm{NaHCO}_{3}(30 \mathrm{~mL})$ was added to the reaction mixture at $0{ }^{\circ} \mathrm{C}$. The solution was diluted with $\mathrm{H}_{2} \mathrm{O}(15 \mathrm{~mL})$ and EtOAc $(75$ $\mathrm{mL})$. The layers were then separated and the aqueous layer was extracted with EtOAc (2 $\mathrm{x} 100 \mathrm{~mL}$ ). The combined organic layers were dried over anhydrous $\mathrm{Na}_{2} \mathrm{SO}_{4}$, filtered and concentrated in vacuo. Purification by flash chromatography (98:2-9:1 hexanesethylacetate) provided triol 14 (354 $\mathrm{mg}, 0.589 \mathrm{mmol}, 92 \%)$ as a white foam.

For triol 14:

$\mathrm{R}_{\mathrm{f}} 0.34$ (6:1 hexanes-EtOAc);

$[\alpha]_{23}^{\mathrm{D}}+33.0\left(\mathrm{c} 0.74, \mathrm{CH}_{2} \mathrm{Cl}_{2}\right)$;

${ }^{1} \mathrm{H}-\mathrm{NMR}\left(400 \mathrm{MHz}, \mathrm{CDCl}_{3}\right) \delta 4.21-4.16(\mathrm{~m}, 1 \mathrm{H}), 4.04$ (bs, $\left.1 \mathrm{H}\right), 3.84$ (ddd, $J=10.2$, $10.2,5.7 \mathrm{~Hz}, 1 \mathrm{H}), 3.64(\mathrm{dd}, J=12.3,4.8 \mathrm{~Hz}, 1 \mathrm{H}), 3.50(\mathrm{dd}, J=11.2,11.2 \mathrm{~Hz}, 1 \mathrm{H}), 3.40$ (ddd, $J=11.0,8.0,3.5 \mathrm{~Hz}, 1 \mathrm{H}), 2.59$ (dd, $J=10.7,4.8 \mathrm{~Hz}, 1 \mathrm{H}), 1.99-0.84$ (m, 25H), $1.01(\mathrm{~d}, J=6.8 \mathrm{~Hz}, 3 \mathrm{H}), 0.99(\mathrm{~s}, 3 \mathrm{H}), 0.94(\mathrm{~s}, 9 \mathrm{H}), 0.91(\mathrm{~s}, 9 \mathrm{H}), 0.14(\mathrm{~s}, 3 \mathrm{H}), 0.13$ (s, $3 \mathrm{H}), 0.13(\mathrm{~s}, 3 \mathrm{H}), 0.09(\mathrm{~s}, 3 \mathrm{H})$;

${ }^{13} \mathrm{C}-\mathrm{NMR}\left(100 \mathrm{MHz}, \mathrm{CDCl}_{3}\right) \delta 72.2,71.6,71.5,69.9,66.8,48.4,43.3,43.1,42.3,38.3$, $35.0,35.0,32.2,31.7,29.9,29.3,26.6,26.5,22.9,21.7,19.5,19.0,18.5,16.1,14.3,-3.1$, $-3.3,-3.6,-3.7$;

IR (film) 3455, 2956, 2929, 2858, 1472, 1388, 1255, 1095, 1044, 983, 906, 836, 812, $775,674 \mathrm{~cm}^{-1}$;

HRMS (ESI+) calc. for $\mathrm{C}_{33} \mathrm{H}_{68} \mathrm{O}_{5} \mathrm{Si}_{2} \mathrm{Na}[\mathrm{M}+\mathrm{Na}]^{+}$623.4497. Found 623.4504. 


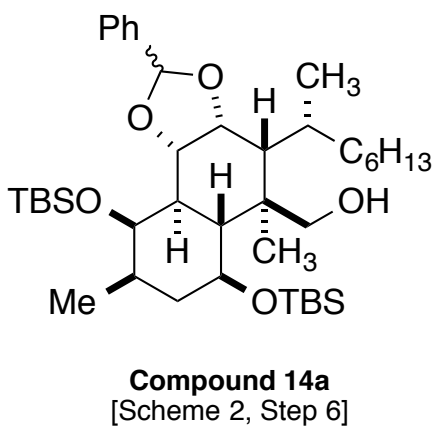

Benzyl acetal 14a: To a $25 \mathrm{~mL}$ round-bottom flask containing triol 14 under argon was added $\mathrm{CH}_{2} \mathrm{Cl}_{2}(7.2 \mathrm{~mL}, 0.02 \mathrm{M}), 4 \AA$ molecular sieves $(260 \mathrm{mg})$, benzaldehyde dimethyl acetal $(0.064 \mathrm{~mL}, 0.429 \mathrm{mmol})$ and PTSA $\bullet \mathrm{H}_{2} \mathrm{O}(8.0 \mathrm{mg}, 0.043 \mathrm{mmol})$. The reaction mixture was stirred at room temperature until no starting material remained by TLC, approximately 13 minutes. A saturated solution of $\mathrm{NaHCO}_{3}(10 \mathrm{~mL})$ was added to the reaction. The solution was diluted with $\mathrm{H}_{2} \mathrm{O}(15 \mathrm{~mL})$ and EtOAc $(50 \mathrm{~mL})$. The layers were then separated and the aqueous layer was extracted with EtOAc $(2 \times 75 \mathrm{~mL})$. The combined organic layers were dried over anhydrous $\mathrm{Na}_{2} \mathrm{SO}_{4}$, filtered and concentrated in vacuo. Purification by flash chromatography (98:2-95:5 hexanes-ethyl acetate) provided benzyl acetal 14a (95 mg, $0.138 \mathrm{mmol}, 96 \%$ ) as a viscous colorless oil.

For 1:1.6 diastereomeric mixture of benzyl acetal 14a:

$\mathrm{R}_{\mathrm{f}} 0.61(6: 1$ hexanes-EtOAc);

$[\alpha]_{23}^{\mathrm{D}}+11.7\left(\mathrm{c} 0.78, \mathrm{CH}_{2} \mathrm{Cl}_{2}\right)$;

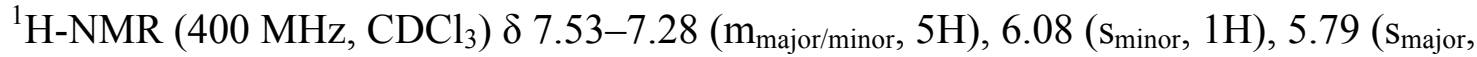
$1 \mathrm{H}), 4.43\left(\mathrm{dd}_{\text {major }}, J=5.5,3.2 \mathrm{~Hz}, 1 \mathrm{H}\right), 4.26\left(\mathrm{dd}_{\text {minor }}, J=4.9,3.0 \mathrm{~Hz}, 1 \mathrm{H}\right), 4.18-4.12$ $\left(\mathrm{m}_{\text {minor }}, 1 \mathrm{H}\right), 4.06\left(\mathrm{dd}_{\text {major }}, J=9.3,5.6 \mathrm{~Hz}, 1 \mathrm{H}\right), 3.99\left(\mathrm{bs}_{\text {minor }}, 1 \mathrm{H}\right), 3.94-3.85\left(\mathrm{~m}_{\text {minor }}, 1 \mathrm{H}\right)$, 3.87 ( $\left.\mathrm{bs}_{\text {major }}, 1 \mathrm{H}\right), 3.83$ (ddd major $\left., J=10.6,9.1,6.0 \mathrm{~Hz}, 1 \mathrm{H}\right), 3.63-3.45\left(\mathrm{~m}_{\text {major } / \text { minor }}, 2 \mathrm{H}\right)$, 3.14-3.02 ( $\left.\mathrm{m}_{\text {major/minor }}, 1 \mathrm{H}\right), 2.17-1.96\left(\mathrm{~m}_{\text {major/minor }}, 1 \mathrm{H}\right), 1.93-0.82\left(\mathrm{~m}_{\text {major } / \text { minor }}, 22 \mathrm{H}\right), 1.10$ $\left(\mathrm{d}_{\text {major }}, J=6.8 \mathrm{~Hz}, 3 \mathrm{H}\right), 1.02\left(\mathrm{~s}_{\text {major }}, 3 \mathrm{H}\right), 0.96\left(\mathrm{~s}_{\text {major }}, 9 \mathrm{H}\right), 0.96\left(\mathrm{~s}_{\text {minor }}, 9 \mathrm{H}\right), 0.93\left(\mathrm{~s}_{\text {minor }}\right.$, $9 \mathrm{H}), 0.92\left(\mathrm{~s}_{\text {major }}, 9 \mathrm{H}\right), 0.21\left(\mathrm{~s}_{\text {minor }}, 3 \mathrm{H}\right), 0.20\left(\mathrm{~s}_{\text {major }}, 3 \mathrm{H}\right), 0.17\left(\mathrm{~s}_{\text {major }}, 3 \mathrm{H}\right), 0.15$ ( $\mathrm{s}_{\text {major }}$, $3 \mathrm{H}), 0.15\left(\mathrm{~s}_{\text {minor }}, 3 \mathrm{H}\right), 0.15\left(\mathrm{~s}_{\text {minor }}, 3 \mathrm{H}\right), 0.11\left(\mathrm{~s}_{\text {minor }}, 3 \mathrm{H}\right), 0.08\left(\mathrm{~s}_{\text {major }}, 3 \mathrm{H}\right)$;

${ }^{13} \mathrm{C}-\mathrm{NMR}\left(125 \mathrm{MHz}, \mathrm{CDCl}_{3}\right) \delta 141.2,139.5,128.8,128.4,128.3,126.7,126.1,103.6$, 101.7, 77.9, 76.3, 75.2, 74.6, 71.6, 71.5, 69.9, 66.1, 46.6, 46.5, 44.9, 43.9, 43.6, 42.5, $41.9,37.7,37.6,34.9,34.8,34.4,34.3,32.4,32.2,32.1,31.9,29.9,29.8,29.1,26.6,26.5$, $22.9,21.3,21.1,19.6,18.9,18.6,15.9,15.5,14.4,-3.1,-3.2,-3.3,-3.5,-3.6$;

IR (film) 3534, 2955, 2929, 2858, 1463, 1388, 1362, 1257, 1218, 1160, 1093, 1051, $1005,987,875,836,812,774,723,692,680,634 \mathrm{~cm}^{-1}$;

HRMS (ESI+) calc. for $\mathrm{C}_{40} \mathrm{H}_{72} \mathrm{O}_{5} \mathrm{Si}_{2} \mathrm{Na}[\mathrm{M}+\mathrm{Na}]^{+}$711.4810. Found 711.4821. 


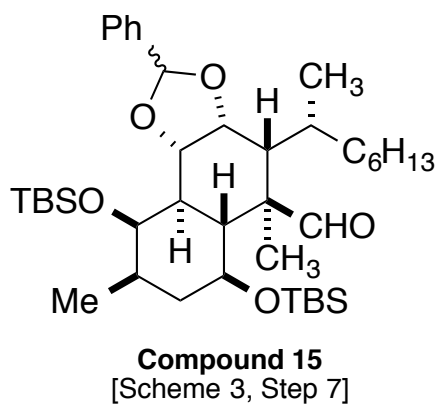

Aldehyde 15: To a $25 \mathrm{~mL}$ round-bottom flask containing benzyl acetal 14a (173 $\mathrm{mg}$, $0.251 \mathrm{mmol})$ under argon was added $\mathrm{CH}_{2} \mathrm{Cl}_{2}(5.0 \mathrm{~mL}, 0.05 \mathrm{M})$ and powdered $4 \AA$ molecular sieves $(270 \mathrm{mg})$. The reaction mixture was cooled to $0{ }^{\circ} \mathrm{C}$, followed by addition of NMO (60 mg, $0.512 \mathrm{mmol})$ and TPAP (10 mg, $0.244 \mathrm{mmol})$. The solution was warmed to room temperature and stirred for $24 \mathrm{~h}$. The reaction mixture was diluted with EtOAc and filtered through a plug of silica gel using EtOAc as the eluent and concentrated in vacuo. The crude NMR still showed the presence of alcohol, therefore the crude mixture was resubjected under the same conditions as previously described. The resulting residue was then purified by flash chromatography (98:2-95:5 hexanes-ethyl acetate) to provide aldehyde 15 (168 $\mathrm{mg}, 0.244 \mathrm{mmol}, 97 \%)$ as a viscous colorless oil.

For 1:1.6 diastereomeric mixture of aldehyde 15:

$\mathrm{R}_{\mathrm{f}} 0.56$ \& 0.48 (95:5 hexanes-EtOAc; developed 2x);

$[\alpha]_{23}^{\mathrm{D}}+16.6\left(\mathrm{c} 0.93, \mathrm{CH}_{2} \mathrm{Cl}_{2}\right)$;

${ }^{1} \mathrm{H}-\mathrm{NMR}\left(500 \mathrm{MHz}, \mathrm{CDCl}_{3}\right) \delta 9.42\left(\mathrm{~s}_{\text {major }}, 1 \mathrm{H}\right), 9.41\left(\mathrm{~s}_{\text {minor }}, 1 \mathrm{H}\right), 7.54-7.30\left(\mathrm{~m}_{\text {major/minor }}\right.$, $5 \mathrm{H}), 6.09\left(\mathrm{~s}_{\text {minor }}, 1 \mathrm{H}\right), 5.84\left(\mathrm{~s}_{\text {major }}, 1 \mathrm{H}\right), 4.40\left(\mathrm{dd}_{\text {major }}, J=5.3,2.9 \mathrm{~Hz}, 1 \mathrm{H}\right), 4.28\left(\mathrm{dd}_{\text {minor }}, J\right.$ $=9.3,4.8 \mathrm{~Hz}, 1 \mathrm{H}), 4.23\left(\mathrm{dd}_{\text {minor }}, J=4.7,2.7 \mathrm{~Hz}, 1 \mathrm{H}\right), 4.14\left(\mathrm{dd}_{\text {major }}, J=9.2,5.3 \mathrm{~Hz}, 1 \mathrm{H}\right)$, $3.99\left(\mathrm{bs}_{\text {minor }}, 1 \mathrm{H}\right), 3.87\left(\mathrm{bs}_{\text {major }}, 1 \mathrm{H}\right), 3.64\left(\mathrm{ddd}_{\text {minor }}, J=9.7,9.7,4.7 \mathrm{~Hz}, 1 \mathrm{H}\right), 3.55$ $\left(\mathrm{ddd}_{\text {major }}, J=10.2,10.2,4.7 \mathrm{~Hz}, 1 \mathrm{H}\right), 1.98-1.90\left(\mathrm{~m}_{\text {major/minor }}, 1 \mathrm{H}\right), 1.80-0.84\left(\mathrm{~m}_{\text {major/minor }}\right.$, $22 \mathrm{H}), 1.29$ ( $\left.\mathrm{s}_{\text {minor }}, 3 \mathrm{H}\right), 1.23$ ( $\left.\mathrm{s}_{\text {major }}, 3 \mathrm{H}\right), 0.97$ ( $\left.\mathrm{s}_{\text {major/minor }}, 9 \mathrm{H}\right), 0.89\left(\mathrm{~s}_{\text {minor }}, 9 \mathrm{H}\right), 0.87$ $\left(\mathrm{s}_{\text {major }}, 9 \mathrm{H}\right), 0.82\left(\mathrm{~d}_{\text {major }}, J=6.6 \mathrm{~Hz}, 1 \mathrm{H}\right), 0.75\left(\mathrm{~d}_{\text {minor }}, J=6.6 \mathrm{~Hz}, 1 \mathrm{H}\right), 0.21\left(\mathrm{~s}_{\text {minor }}, 3 \mathrm{H}\right)$, $0.19\left(\mathrm{~s}_{\text {major }}, 3 \mathrm{H}\right), 0.12\left(\mathrm{~s}_{\text {minor }}, 3 \mathrm{H}\right), 0.09\left(\mathrm{~s}_{\text {major }}, 3 \mathrm{H}\right), 0.08\left(\mathrm{~s}_{\text {minor }}, 3 \mathrm{H}\right), 0.07\left(\mathrm{~s}_{\text {minor }}, 3 \mathrm{H}\right)$, 0.05 ( $\left.\mathrm{s}_{\text {major }}, 3 \mathrm{H}\right), 0.05\left(\mathrm{~s}_{\text {major }}, 3 \mathrm{H}\right)$;

${ }^{13} \mathrm{C}-\mathrm{NMR}\left(125 \mathrm{MHz}, \mathrm{CDCl}_{3}\right) \delta 205.3,140.5,139.0,128.8,128.4,128.3,128.1,126.4$, $125.9,103.6,101.6,76.5,75.3,74.0,73.2,72.3,72.2,70.0,69.9,49.8,47.8,47.6,45.6$, $45.5,44.1,41.4,37.2,37.1,35.6,35.5,34.1,34.0,32.0,31.9,31.8,29.7,29.6,27.4,27.3$, $26.4,26.3,26.1,22.7,21.4,19.4,18.7,18.2,14.1,11.5,11.3,-3.0,-3.3,-3.4,-3.9$;

IR (film) 2955, 2929, 2857, 1727, 1471, 1463, 1407, 1384, 1361, 1254, 1218, 1155 , $1137,1095,1065,1006,992,940,909,870,837,812,776,724,696 \mathrm{~cm}^{-1}$;

HRMS (ESI+) calc. for $\mathrm{C}_{40} \mathrm{H}_{70} \mathrm{O}_{5} \mathrm{Si}_{2} \mathrm{Na}[\mathrm{M}+\mathrm{Na}]^{+}$709.4654. Found 709.4651. 


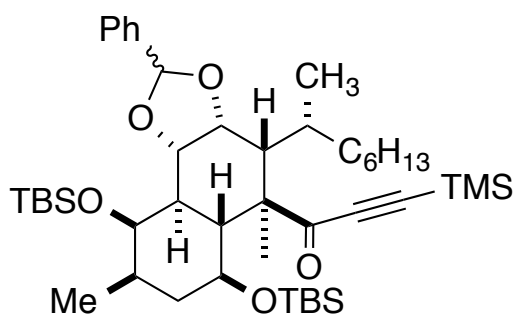

Compound 16

[Scheme 3, Step 9]

TMS-Ynone 16: To a flame-dried $50 \mathrm{~mL}$ round-bottom flask charged with a magnetic stir bar, evacuated and back-filled with argon was added trimethylsilyl acetylene (1.04 $\mathrm{mL}, 7.32 \mathrm{mmol})$ and THF $(7.3 \mathrm{~mL})$. The solution was cooled to $-78^{\circ} \mathrm{C}$, followed by the addition of $n$-BuLi $(3.47 \mathrm{~mL}, 6.11 \mathrm{mmol}, 1.76 \mathrm{M})$ and the reaction was allowed to stir for $40 \mathrm{~min}$ at $-78^{\circ} \mathrm{C}$. A THF $(4.0 \mathrm{~mL})$ solution of aldehyde $15(168 \mathrm{mg}, 0.244 \mathrm{mmol})$ was slowly added down the sides of the flask to the TMS lithium acetylide solution at -78 ${ }^{\circ} \mathrm{C}$. The cooling bath was removed and the reaction mixture was allowed to warm to room temperature and stirred for $18 \mathrm{~h}$. A saturated solution of $\mathrm{NH}_{4} \mathrm{Cl}(15 \mathrm{~mL})$ was added to the reaction at $0{ }^{\circ} \mathrm{C}$. The solution was diluted with $\mathrm{H}_{2} \mathrm{O}(25 \mathrm{~mL})$ and EtOAc $(75 \mathrm{~mL})$. The layers were then separated and the aqueous layer was extracted with EtOAc $(2 \times 75$ $\mathrm{mL}$ ). The combined organic layers were dried over anhydrous $\mathrm{Na}_{2} \mathrm{SO}_{4}$, filtered and concentrated in vacuo to provide a yellow oil. The crude product was used in the following reaction without purification. To a $50 \mathrm{~mL}$ round-bottom flask containing crude alcohol under argon was added $\mathrm{CH}_{2} \mathrm{Cl}_{2}(12.2 \mathrm{~mL}, 0.02 \mathrm{M})$. The reaction was cooled to 0 ${ }^{\circ} \mathrm{C}$, followed by the addition of $\mathrm{NaHCO}_{3}(210 \mathrm{mg}, 2.44 \mathrm{mmol})$ and Dess-Martin periodinane $(311 \mathrm{mg}, 0.732 \mathrm{mmol})$ to the reaction mixture. The solution was warmed to room temperature and stirred for $20 \mathrm{~h}$. The mixture was cooled to $0{ }^{\circ} \mathrm{C}$ and saturated $\mathrm{NaHCO}_{3}(15 \mathrm{~mL})$ and saturated $\mathrm{Na}_{2} \mathrm{~S}_{2} \mathrm{O}_{3}(20 \mathrm{~mL})$ were added. The mixture was warmed to room temperature and stirred for $15 \mathrm{~min}$. The solution was diluted with $\mathrm{H}_{2} \mathrm{O}(15 \mathrm{~mL})$ and $\mathrm{Et}_{2} \mathrm{O}(70 \mathrm{~mL})$. The layers were then separated and the aqueous layer was extracted with $\mathrm{Et}_{2} \mathrm{O}(2 \times 75 \mathrm{~mL})$. The combined organic layers were dried over anhydrous $\mathrm{Na}_{2} \mathrm{SO}_{4}$, filtered and concentrated in vacuo. Purification by flash chromatography (98:2-95:5 hexanes-ethyl acetate) provided TMS-ynone 16 (175 mg, $0.223 \mathrm{mmol}, 92 \%$ over 2 steps) as a viscous colorless oil.

For 1:1.3 diastereomeric mixture of TMS-ynone 16:

$\mathrm{R}_{\mathrm{f}} 0.63$ \& 0.57 (95:5 hexanes-EtOAc; developed 2x);

$[\alpha]_{23}^{\mathrm{D}}+30.4\left(\mathrm{c} 0.57, \mathrm{CH}_{2} \mathrm{Cl}_{2}\right)$;

${ }^{1} \mathrm{H}-\mathrm{NMR}\left(400 \mathrm{MHz}, \mathrm{CDCl}_{3}\right) \delta$ 7.47-7.30 (m $\left.\mathrm{m}_{\text {major/minor }}, 5 \mathrm{H}\right), 6.09$ ( $\left.\mathrm{s}_{\text {minor }}, 1 \mathrm{H}\right), 5.83$ ( $\mathrm{s}_{\text {major }}$, $1 \mathrm{H}), 4.40\left(\mathrm{dd}_{\text {major }}, J=5.2,3.0 \mathrm{~Hz}, 1 \mathrm{H}\right), 4.29\left(\mathrm{dd}_{\text {minor }}, J=9.4,4.6 \mathrm{~Hz}, 1 \mathrm{H}\right), 4.22\left(\mathrm{dd}_{\text {minor }}, J\right.$ $=4.7,2.7 \mathrm{~Hz}, 1 \mathrm{H}), 4.18\left(\mathrm{dd}_{\text {major }}, J=9.1,5.1 \mathrm{~Hz}, 1 \mathrm{H}\right), 4.01$ (bs minor $\left._{1}, 1 \mathrm{H}\right), 3.89$ (bs $_{\text {major, }}$, $1 \mathrm{H}), 3.56\left(\mathrm{ddd}_{\text {minor }}, J=10.2,10.2,4.4 \mathrm{~Hz}, 1 \mathrm{H}\right), 3.46\left(\mathrm{ddd}_{\text {major }}, J=10.3,10.3,4.5 \mathrm{~Hz}\right.$, $1 \mathrm{H}), 2.52-2.41\left(\mathrm{~m}_{\text {major/minor }}, 1 \mathrm{H}\right), 2.04\left(\mathrm{dd}_{\text {major }}, J=2.9,2.9 \mathrm{~Hz}, 1 \mathrm{H}\right), 1.94\left(\mathrm{dd}_{\text {minor }}, J=2.3\right.$, $2.3 \mathrm{~Hz}, 1 \mathrm{H}), 1.85-1.75\left(\mathrm{~m}_{\text {major/minor }}, 1 \mathrm{H}\right), 1.70-0.81\left(\mathrm{~m}_{\text {major/minor }}, 23 \mathrm{H}\right), 1.26\left(\mathrm{~s}_{\text {minor }}, 3 \mathrm{H}\right)$, 
$1.20\left(\mathrm{~s}_{\text {major }}, 3 \mathrm{H}\right), 1.00\left(\mathrm{~s}_{\text {major }}, 9 \mathrm{H}\right), 0.99\left(\mathrm{~s}_{\text {minor }}, 9 \mathrm{H}\right), 0.89\left(\mathrm{~s}_{\text {minor }}, 9 \mathrm{H}\right), 0.87\left(\mathrm{~s}_{\text {major }}, 9 \mathrm{H}\right), 0.23$

$\left(\mathrm{s}_{\text {minor }}, 3 \mathrm{H}\right), 0.22\left(\mathrm{~s}_{\text {major }}, 3 \mathrm{H}\right), 0.21\left(\mathrm{~s}_{\text {major }}, 9 \mathrm{H}\right), 0.19\left(\mathrm{~s}_{\text {minor }}, 9 \mathrm{H}\right), 0.13\left(\mathrm{~s}_{\text {minor }}, 3 \mathrm{H}\right), 0.11$

$\left(\mathrm{s}_{\text {major }}, 3 \mathrm{H}\right), 0.06\left(\mathrm{~s}_{\text {minor }}, 3 \mathrm{H}\right), 0.03\left(\mathrm{~s}_{\text {minor }}, 3 \mathrm{H}\right), 0.02\left(\mathrm{~s}_{\text {major }}, 3 \mathrm{H}\right), 0.00\left(\mathrm{~s}_{\text {major }}, 3 \mathrm{H}\right)$;

${ }^{13} \mathrm{C}-\mathrm{NMR}\left(125 \mathrm{MHz}, \mathrm{CDCl}_{3}\right) \delta 191.5,140.6,139.1,128.7,128.3,128.1,126.3,125.9$, $103.8,101.9,101.8,96.9,75.8,75.7,74.4,72.6,72.1,71.9,70.1,70.0,53.6,47.0,46.9$, $46.8,46.6,44.8,42.3,37.6,37.5,35.5,34.6,32.6,32.1,32.0,29.7,29.6,28.5,26.5,26.3$, $22.7,22.6,21.3,19.6,19.5,18.7,18.2,14.2,11.4,11.3,-0.6,-2.8,-3.7,-3.8,-4.1$;

IR (film) 2956, 2928, 2857, 1672, 1463, 1407, 1385, 1360, 1252, 1155, 1133, 1096, $1179,1067,1005,940,840,812,775,697,677,629,574 \mathrm{~cm}^{-1}$;

HRMS (ESI+) calc. for $\mathrm{C}_{45} \mathrm{H}_{78} \mathrm{O}_{5} \mathrm{Si}_{2} \mathrm{Na}[\mathrm{M}+\mathrm{Na}]^{+}$805.5049. Found 805.5090. 


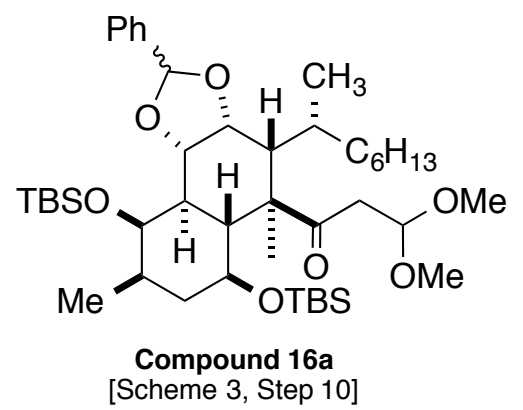

Dimethyl Acetal 16a: To a culture tube containing TMS-ynone 16 (98 mg, $0.125 \mathrm{mmol})$ under argon was added anhydrous $\mathrm{MeOH}(6.3 \mathrm{~mL}, 0.02 \mathrm{M})$ and $\mathrm{K}_{2} \mathrm{CO}_{3}(346 \mathrm{mg}, 2.50$ mmol). The reaction was heated to $65^{\circ} \mathrm{C}$ and stirred for $20 \mathrm{~h}$. The solution was cooled to room temperature and diluted with $\mathrm{H}_{2} \mathrm{O}(50 \mathrm{~mL})$ and EtOAc $(100 \mathrm{~mL})$. The layers were then separated and the aqueous layer was extracted with EtOAc $(2 \times 100 \mathrm{~mL})$. The combined organic layers were washed with $\mathrm{H}_{2} \mathrm{O}(1 \times 50 \mathrm{~mL})$, dried over anhydrous $\mathrm{Na}_{2} \mathrm{SO}_{4}$, filtered and concentrated in vacuo. Purification by flash chromatography $(98: 2-$ 95:5 hexanes-ethyl acetate) provided dimethyl acetal $\mathbf{1 6 a}$ (95 $\mathrm{mg}, 0.123 \mathrm{mmol}, 98 \%$ ) as a viscous colorless oil.

For 1:1.6 diastereomeric mixture of dimethyl acetal 16a:

$\mathrm{R}_{\mathrm{f}} 0.67$ (6:1 hexanes-EtOAc);

$[\alpha]_{23}^{\mathrm{D}}+28.9\left(\mathrm{c} 0.71, \mathrm{CH}_{2} \mathrm{Cl}_{2}\right)$;

${ }^{1} \mathrm{H}-\mathrm{NMR}\left(400 \mathrm{MHz}, \mathrm{CDCl}_{3}\right) \delta$ 7.49-7.30 (m $\left.\mathrm{m}_{\text {major/minor }}, 5 \mathrm{H}\right), 6.08\left(\mathrm{~s}_{\text {minor }}, 1 \mathrm{H}\right), 5.84$ ( $\mathrm{s}_{\text {major }}$, $1 \mathrm{H}), 4.97-4.92\left(\mathrm{~m}_{\text {major/minor }}, 1 \mathrm{H}\right), 4.40\left(\mathrm{dd}_{\text {major }}, J=5.0,2.8 \mathrm{~Hz}, 1 \mathrm{H}\right), 4.28-4.21\left(\mathrm{~m}_{\text {minor }}\right.$, $1 \mathrm{H}), 4.28-4.21\left(\mathrm{~m}_{\text {minor }}, 1 \mathrm{H}\right), 4.13\left(\mathrm{dd}_{\text {major }}, J=9.1,5.1 \mathrm{~Hz}, 1 \mathrm{H}\right), 4.01$ ( $\left.\mathrm{bs}_{\text {minor }}, 1 \mathrm{H}\right), 3.89$ (bs $\left.\mathrm{bajor}_{\text {moj }}, 1 \mathrm{H}\right), 3.63\left(\mathrm{~m}_{\text {minor }}, 1 \mathrm{H}\right), 3.50-3.38\left(\mathrm{~m}_{\text {major }}, 1 \mathrm{H}\right), 3.41\left(\mathrm{~s}_{\text {major }}, 3 \mathrm{H}\right), 3.39\left(\mathrm{~s}_{\text {minor }}, 3 \mathrm{H}\right)$, $3.33\left(\mathrm{~s}_{\text {major }}, 3 \mathrm{H}\right), 3.32\left(\mathrm{~s}_{\text {minor }}, 3 \mathrm{H}\right), 3.00-2.88\left(\mathrm{~m}_{\text {major/minor }}, 1 \mathrm{H}\right), 2.88-2.79\left(\mathrm{~m}_{\text {major/minor }}, 1 \mathrm{H}\right)$, $2.30-2.19\left(\mathrm{~m}_{\text {major/minor }}, 1 \mathrm{H}\right), 1.92-1.79\left(\mathrm{~m}_{\text {major/minor }}, 1 \mathrm{H}\right), 1.71-0.79\left(\mathrm{~m}_{\text {major/minor }}, 24 \mathrm{H}\right), 1.28$ ( $\left.\mathrm{s}_{\text {minor }}, 3 \mathrm{H}\right), 1.23$ ( $\left.\mathrm{s}_{\text {major }}, 3 \mathrm{H}\right), 0.99$ ( $\left.\mathrm{s}_{\text {major }}, 9 \mathrm{H}\right), 0.98$ ( $\left.\mathrm{s}_{\text {minor }}, 9 \mathrm{H}\right), 0.87$ ( $\left.\mathrm{s}_{\text {minor }}, 9 \mathrm{H}\right), 0.85$ $\left(\mathrm{s}_{\text {major }}, 9 \mathrm{H}\right), 0.21\left(\mathrm{~s}_{\text {major/minor }}, 3 \mathrm{H}\right), 0.13\left(\mathrm{~s}_{\text {minor }}, 3 \mathrm{H}\right), 0.11\left(\mathrm{~s}_{\text {major }}, 3 \mathrm{H}\right), 0.05\left(\mathrm{~s}_{\text {minor }}, 3 \mathrm{H}\right), 0.05$ $\left(\mathrm{s}_{\text {minor }}, 3 \mathrm{H}\right), 0.02\left(\mathrm{~s}_{\text {major }}, 3 \mathrm{H}\right), 0.02\left(\mathrm{~s}_{\text {major }}, 3 \mathrm{H}\right)$;

${ }^{13} \mathrm{C}-\mathrm{NMR}\left(100 \mathrm{MHz}, \mathrm{CDCl}_{3}\right) \delta 209.7,209.6,140.7,139.2,128.8,128.5,128.4,128.3$, 126.4, 126.0, 104.0, 103.4, 102.1, 77.4, 76.0, 75.7, 74.7, 73.1, 73.0, 72.6, 70.7, 70.5, 56.5, $56.4,53.6,53.0,46.7,46.6,45.4,44.2,43.9,42.7,41.4,38.6,38.5,35.9,35.8,34.9,34.8$, $32.6,32.5,32.2,32.1,29.9,29.8,29.2,29.1,26.5,26.4,22.8,21.0,20.9,19.5,18.8,18.5$, $18.4,14.3,12.5,12.4,-2.6,-3.7,-3.8,-4.3$;

IR (film) 2955, 2929, 2857, 1708, 1463, 1385, 1294, 1254, 1218, 1154, 1125, 1195 , $1095,1064,1006,947,870,812,777,744,696,671,575 \mathrm{~cm}^{-1}$;

HRMS (ESI+) calc. for $\mathrm{C}_{44} \mathrm{H}_{78} \mathrm{O}_{7} \mathrm{Si}_{2} \mathrm{Na}[\mathrm{M}+\mathrm{Na}]+797.5178$. Found 797.5181. 


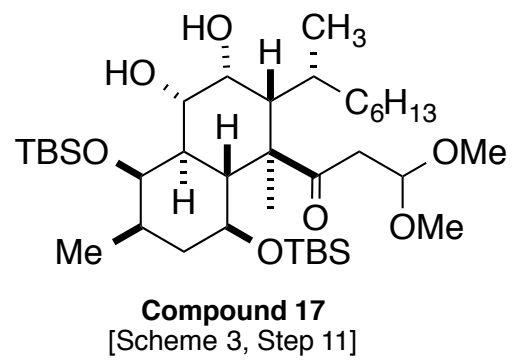

Diol 17: To a $50 \mathrm{~mL}$ round-bottom flask containing benzyl acetal 16a $(95 \mathrm{mg}, 0.123$ mmol) dissolved in EtOAc $(9.4 \mathrm{~mL}, 0.013 \mathrm{M})$ was added $\mathrm{Pd} / \mathrm{C}(206 \mathrm{mg}, 5 \% \mathrm{w} / \mathrm{w})$. The mixture was degassed by applying vacuum and backfilling with argon before charging the flask with hydrogen using vacuum and backfilling from a balloon. The mixture was then stirred vigorously under a hydrogen filled balloon for $18 \mathrm{~h}$. The balloon was removed and the mixture was purged of hydrogen by applying vacuum and back filling with argon several times. The reaction mixture was diluted with EtOAc and filtered through a plug of silica gel using EtOAc as the eluent and concentrated in vacuo. Purification by flash chromatography (95:5-6:1 hexanes-ethyl acetate) provided diol 17 (76 mg, $0.111 \mathrm{mmol}, 90 \%$ ) as a white solid.

For diol 17:

$\mathrm{R}_{\mathrm{f}} 0.38$ (6:1 hexanes-EtOAc);

$[\alpha]_{23}^{\mathrm{D}}+40.4\left(\mathrm{c} 0.75, \mathrm{CH}_{2} \mathrm{Cl}_{2}\right)$;

${ }^{1} \mathrm{H}-\mathrm{NMR}\left(500 \mathrm{MHz}, \mathrm{CDCl}_{3}\right) \delta 4.91(\mathrm{dd}, J=8.2,2.0 \mathrm{~Hz}, 1 \mathrm{H}), 4.19$ (bs, 1H), 4.06 (bs, $1 \mathrm{H}), 3.53(\mathrm{ddd}, J=10.1,10.1,4.9 \mathrm{~Hz}, 1 \mathrm{H}), 3.45$ (ddd, $J=11.2,8.6,3.2 \mathrm{~Hz}, 1 \mathrm{H}), 3.36$ (s, $3 \mathrm{H}), 3.28$ (s, 3H), 2.90 (dd, $J=16.9,8.3 \mathrm{~Hz}, 1 \mathrm{H}), 2.74$ (dd, $J=16.9,1.8 \mathrm{~Hz}, 1 \mathrm{H}), 2.32$ $(\mathrm{dd}, J=11.0,11.0 \mathrm{~Hz}, 1 \mathrm{H}), 2.06(\mathrm{~d}, J=8.6 \mathrm{~Hz}, 1 \mathrm{H}), 1.92(\mathrm{~d}, J=3.1 \mathrm{~Hz}, 1 \mathrm{H}), 1.88$ (ddd, $J=11.2,11.2,4.5 \mathrm{~Hz}, 1 \mathrm{H}), 1.71-0.80(\mathrm{~m}, 24 \mathrm{H}), 0.96(\mathrm{~s}, 9 \mathrm{H}), 0.92(\mathrm{~d}, J=6.7 \mathrm{~Hz}, 3 \mathrm{H})$, $0.83(\mathrm{~s}, 9 \mathrm{H}), 0.15(\mathrm{~s}, 3 \mathrm{H}), 0.09(\mathrm{~s}, 3 \mathrm{H}), 0.02(\mathrm{~s}, 3 \mathrm{H}), 0.01(\mathrm{~s}, 3 \mathrm{H})$;

${ }^{13} \mathrm{C}-\mathrm{NMR}\left(125 \mathrm{MHz}, \mathrm{CDCl}_{3}\right) \delta 209.9,103.2,72.8,71.0,69.8,56.2,54.3,52.5,48.5$, 44.0, 43.7, 41.2, 38.6, 35.7, 35.2, 32.4, 32.1, 29.9, 29.3, 26.5, 26.3, 22.8, 20.9, 19.5, 18.9, $18.4,14.2,13.6,-3.3,-3.8,-3.9,-4.2$;

IR (film) 3483, 2955, 2929, 2858, 1706, 1463, 1380, 1255, 1193, 1125, 1096, 1061, $1038,1006,985,948,885,837,813,777,679 \mathrm{~cm}^{-1}$;

HRMS (ESI+) calc. for $\mathrm{C}_{37} \mathrm{H}_{74} \mathrm{O}_{7} \mathrm{Si}_{2} \mathrm{Na}[\mathrm{M}+\mathrm{Na}]^{+}$709.4865. Found 709.4899. 


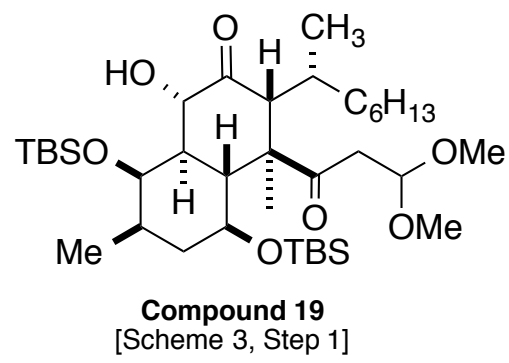

Ketol 19: To a culture tube containing diol 17 (35 mg, $0.051 \mathrm{mmol})$ under argon was added anhydrous DMSO (5.1 mL, $0.01 \mathrm{M})$ and IBX (143 $\mathrm{mg}, 0.509 \mathrm{mmol})$. The reaction was stirred at room temperature for $16 \mathrm{~h}$. The reaction mixture was diluted with $\mathrm{H}_{2} \mathrm{O}(20$ $\mathrm{mL})$ and EtOAc $(50 \mathrm{~mL})$. The layers were then separated and the aqueous layer was extracted with EtOAc $(2 \times 50 \mathrm{~mL})$. The combined organic layers were washed with $\mathrm{H}_{2} \mathrm{O}$ $(2 \times 30 \mathrm{~mL})$, dried over anhydrous $\mathrm{Na}_{2} \mathrm{SO}_{4}$, filtered and concentrated in vacuo.

Purification by flash chromatography (98:2-9:1 hexanes-ethyl acetate) provided ketol 19 (31 $\mathrm{mg}, 0.045 \mathrm{mmol}, 89 \%$ ) as a viscous colorless oil.

\section{For ketol 19:}

$\mathrm{R}_{\mathrm{f}} 0.69$ (4:1 hexanes-EtOAc);

$[\alpha]_{23}^{\mathrm{D}}-2.9\left(\mathrm{c} 0.75, \mathrm{CH}_{2} \mathrm{Cl}_{2}\right)$;

${ }^{1} \mathrm{H}-\mathrm{NMR}\left(500 \mathrm{MHz}, \mathrm{CDCl}_{3}\right) \delta 4.94(\mathrm{dd}, J=8.1,1.9 \mathrm{~Hz}, 1 \mathrm{H}), 4.03(\mathrm{dd}, J=10.4,3.3 \mathrm{~Hz}$, $1 \mathrm{H}), 3.98$ (bs, 1H), 3.57 (d, $J=3.5 \mathrm{~Hz}, 1 \mathrm{H}), 3.47$ (ddd, $J=9.8,9.8,4.7 \mathrm{~Hz}, 1 \mathrm{H}), 3.38$ (s, $3 \mathrm{H}), 3.34$ (s, 3H), 3.05 (dd, $J=17.0,8.2 \mathrm{~Hz}, 1 \mathrm{H}), 2.95$ (dd, $J=17.0,2.0 \mathrm{~Hz}, 1 \mathrm{H}), 2.57$ (bs, 1H), 1.69- $0.79(\mathrm{~m}, 24 \mathrm{H}), 1.01(\mathrm{~s}, 9 \mathrm{H}), 0.94(\mathrm{~d}, J=6.7 \mathrm{~Hz}, 3 \mathrm{H}), 0.86(\mathrm{~s}, 9 \mathrm{H}), 0.15$ $(\mathrm{s}, 3 \mathrm{H}), 0.12(\mathrm{~s}, 3 \mathrm{H}), 0.03(\mathrm{~s}, 3 \mathrm{H}), 0.01(\mathrm{~s}, 3 \mathrm{H})$;

${ }^{13} \mathrm{C}-\mathrm{NMR}\left(125 \mathrm{MHz}, \mathrm{CDCl}_{3}\right) \delta 211.3,205.3,102.9,73.9,72.9,70.8,58.7,57.9,56.2$, 53.7, 53.0, 44.7, 41.2, 38.8, 35.9, 32.9, 32.2, 31.4, 29.7, 29.2, 26.5, 26.3, 22.9, 20.2 , 19.3, $18.8,18.4,14.3,12.1,-3.0,-3.7,-4.3,-4.4$;

IR (film) 3479, 2955, 2929, 2857, 1716, 1463, 1385, 1298, 1254, 1164, 1125, 1061, $1039,1007,988,876 \mathrm{~cm}^{-1}$;

HRMS (ESI+) calc. for $\mathrm{C}_{37} \mathrm{H}_{72} \mathrm{O}_{7} \mathrm{Si}_{2} \mathrm{Na}[\mathrm{M}+\mathrm{Na}]^{+}$707.4709. Found 707.4681. 

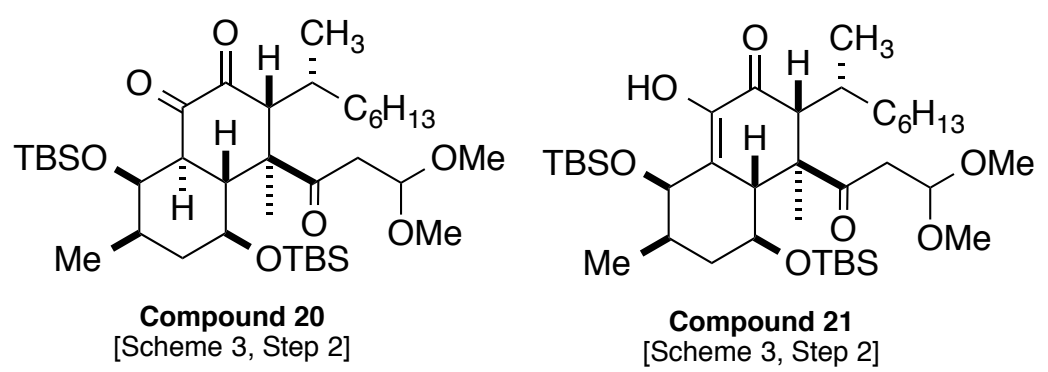

Diketone 20/Keto-Enol 21: To a culture tube containing ketol 19 (15 mg, $0.219 \mathrm{mmol}$ ) under argon was added $\mathrm{CH}_{2} \mathrm{Cl}_{2}(2.7 \mathrm{~mL}, 0.008 \mathrm{M})$, sodium acetate $(23 \mathrm{mg}, 0.280 \mathrm{mmol})$ and powdered $4 \AA$ molecular sieves $(100 \mathrm{mg}$ ). The reaction mixture was stirred for $5 \mathrm{~min}$ and then PCC (23 mg, $0.105 \mathrm{mmol})$ was added. The reaction was stirred until no starting material remained by TLC. The reaction mixture was diluted with $\mathrm{Et}_{2} \mathrm{O}$ and filtered through a plug of silica gel using $\mathrm{Et}_{2} \mathrm{O}$ as the eluent and concentrated in vacuo. The resulting residue was then purified by flash chromatography (98:2-9:1 hexanes-ethyl acetate) to provide keto-enol 21 (12 $\mathrm{mg}, 0.176 \mathrm{mmol}, 80 \%)$ as a colorless oil.

For diketone 20:

$\mathrm{R}_{\mathrm{f}} 0.60$ (6:1 hexanes-EtOAc);

${ }^{1} \mathrm{H}-\mathrm{NMR}\left(400 \mathrm{MHz}, \mathrm{CDCl}_{3}\right) \delta 4.98(\mathrm{dd}, J=6.5,3.6 \mathrm{~Hz}, 1 \mathrm{H}), 4.50$ (bs, $1 \mathrm{H}$ ), 3.57 (ddd, $J$ $=9.4,9.4,3.6 \mathrm{~Hz}, 1 \mathrm{H}), 3.41(\mathrm{~s}, 3 \mathrm{H}), 3.34(\mathrm{~s}, 3 \mathrm{H}), 3.24(\mathrm{dd}, J=11.6,9.8 \mathrm{~Hz}, 1 \mathrm{H}), 3.07-$ $3.01(\mathrm{~m}, 2 \mathrm{H}), 2.82(\mathrm{bs}, 1 \mathrm{H}), 2.16(\mathrm{dd}, J=11.6,1.2 \mathrm{~Hz}, 1 \mathrm{H}), 1.69-0.77(\mathrm{~m}, 26 \mathrm{H}), 0.90$ (s, $9 \mathrm{H}), 0.88(\mathrm{~s}, 9 \mathrm{H}), 0.13(\mathrm{~s}, 3 \mathrm{H}), 0.07$ (s, 3H), $0.05(\mathrm{~s}, 3 \mathrm{H}),-0.02(\mathrm{~s}, 3 \mathrm{H})$.

For keto-enol 21:

$\mathrm{R}_{\mathrm{f}} 0.60$ (6:1 hexanes-EtOAc);

$[\alpha]_{23}^{\mathrm{D}}-7.1\left(\mathrm{c} 0.38, \mathrm{CH}_{2} \mathrm{Cl}_{2}\right)$;

${ }^{1} \mathrm{H}-\mathrm{NMR}\left(500 \mathrm{MHz}, \mathrm{CDCl}_{3}\right) \delta 6.23(\mathrm{~s}, 1 \mathrm{H}), 4.93(\mathrm{dd}, J=7.7,2.3 \mathrm{~Hz}, 1 \mathrm{H}), 4.72(\mathrm{~d}, J=$ $1.9 \mathrm{~Hz}, 1 \mathrm{H}), 3.63(\mathrm{ddd}, J=10.9,10.9,3.7 \mathrm{~Hz}, 1 \mathrm{H}), 3.40(\mathrm{~s}, 3 \mathrm{H}), 3.36(\mathrm{~s}, 3 \mathrm{H}), 3.27$ (d, $J=$ $10.0 \mathrm{~Hz}, 1 \mathrm{H}), 3.00(\mathrm{dd}, J=16.9,7.8 \mathrm{~Hz}, 1 \mathrm{H}), 2.88(\mathrm{dd}, J=17.0,2.4 \mathrm{~Hz}, 1 \mathrm{H}), 2.60(\mathrm{~s}$, $1 \mathrm{H}), 1.78-0.84(\mathrm{~m}, 27 \mathrm{H}), 1.15(\mathrm{~s}, 3 \mathrm{H}), 1.05(\mathrm{~d}, J=6.9 \mathrm{~Hz}, 3 \mathrm{H}), 0.98(\mathrm{~d}, J=6.6 \mathrm{~Hz}, 3 \mathrm{H})$, 0.90 (s, 9H), 0.87 (s, 9H), 0.07 (s, 3H), 0.06 (s, 3H), 0.05 (s, 3H), -0.06 (s, 3H);

13C-NMR (125 MHz, $\left.\mathrm{CDCl}_{3}\right) \delta 206.9,194.9,141.6,125.3,103.2,72.6,66.9,56.2,54.6$, 53.8, 46.0, 42.4, 37.2, 34.3, 33.5, 32.1, 31.8, 29.9, 29.8, 29.4, 26.3, 25.9, 22.9, 21.9, 18.5, $18.4,17.8,14.3,14.2,-3.6,-3.9,-4.7,-5.1$

IR (film) 3411, 2955, 2929, 2857, 1712, 1685, 1663, 1463, 1377, 1289, 1258, 1201, $1124,1090,1056,1028,938,889,836,814,778,720,666 \mathrm{~cm}^{-1}$;

HRMS (ESI+) calc. for $\mathrm{C}_{37} \mathrm{H}_{70} \mathrm{O}_{7} \mathrm{Si}_{2} \mathrm{Na}[\mathrm{M}+\mathrm{Na}]^{+}$705.4552. Found 705.4540. 


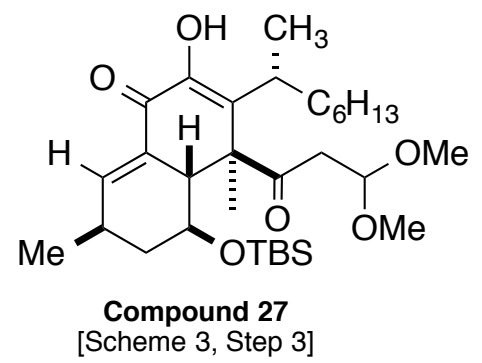

Keto-enol 27: To a plastic culture tube containing keto-enol 21 (4 mg, $0.0059 \mathrm{mmol})$ was added THF $(0.700 \mathrm{~mL}, 0.008 \mathrm{M})$. The reaction was cooled to $0{ }^{\circ} \mathrm{C}$ and $\mathrm{HF} \bullet$ pyridine $(0.050 \mathrm{~mL}$ of a $70 \% \mathrm{HF} / 30 \%$ pyridine $)$ was added dropwise. The reaction was stirred for $72 \mathrm{~h}$ at room temperature. A saturated solution of $\mathrm{NaHCO}_{3}(5 \mathrm{~mL})$ was added to the reaction mixture at $0{ }^{\circ} \mathrm{C}$. The solution was diluted with $\mathrm{H}_{2} \mathrm{O}(10 \mathrm{~mL})$ and EtOAc $(30$ $\mathrm{mL}$ ). The layers were then separated and the aqueous layer was extracted with EtOAc (2 x $30 \mathrm{~mL}$ ). The combined organic layers were dried over anhydrous $\mathrm{Na}_{2} \mathrm{SO}_{4}$, filtered and concentrated in vacuo. Purification by flash chromatography $(9: 1-6: 1$ hexanes-ethyl acetate) provided keto-enol 27 (1.0 $\mathrm{mg}, 0.0018 \mathrm{mmol}, 31 \%$; at 50\% conversion) as a colorless oil.

For keto-enol 27:

$\mathrm{R}_{\mathrm{f}} 0.55$ (6:1 hexanes-EtOAc);

${ }^{1} \mathrm{H}-\mathrm{NMR}\left(400 \mathrm{MHz}, \mathrm{CDCl}_{3}\right) \delta$ 6.97-6.94 (m, 1H), 6.59 (s, 1H), $4.91(\mathrm{dd}, J=7.1,3.1 \mathrm{~Hz}$, $1 \mathrm{H}), 3.93$ (ddd, $J=11.8,9.0,3.2 \mathrm{~Hz}, 1 \mathrm{H}), 3.43(\mathrm{~s}, 3 \mathrm{H}), 3.36(\mathrm{~s}, 3 \mathrm{H}), 3.27-3.20(\mathrm{~m}, 1 \mathrm{H})$, 3.09 (dd, $J=17.7,3.1 \mathrm{~Hz}, 1 \mathrm{H}), 2.97$ (dd, $J=17.6,7.2 \mathrm{~Hz}, 1 \mathrm{H}), 2.53-2.40$ (m, 1H), 2.02 (ddd, $J=13.0,4.4,4.4 \mathrm{~Hz}, 1 \mathrm{H}), 1.95-1.78(\mathrm{~m}, 2 \mathrm{H}), 1.43-1.04(\mathrm{~m}, 16 \mathrm{H}), 1.13(\mathrm{~d}, J=7.2$ $\mathrm{Hz}, 3 \mathrm{H}), 0.88$ (s, 9H), 0.87 (t, $7.1 \mathrm{~Hz}, 3 \mathrm{H}), 0.09$ (s, 3H), 0.07 (s, 3H);

HRMS (ESI+) calc. for $\mathrm{C}_{31} \mathrm{H}_{54} \mathrm{O}_{6} \mathrm{SiNa}[\mathrm{M}+\mathrm{Na}]^{+}$573.3582. Found 573.3560. 


\begin{tabular}{|lll|}
\hline \multicolumn{1}{|c|}{ Parameter } & \multicolumn{1}{c|}{ Value } \\
1 & Title & jck-3-295 \\
2 & Origin & Varian \\
3 & Spectrometer & inova \\
4 & Author & jcklein \\
5 & Solvent & cdcl3 \\
6 & Temperature & 25.0 \\
7 & Pulse Sequence & s2pul \\
8 & Number of Scans & 16 \\
9 & Receiver Gain & 48 \\
10 & Relaxation Delay & 1.0000 \\
11 & Pulse Width & 0.0000 \\
12 & Acquisition Time & 2.0031 \\
13 & Acquisition Date & $2006-11-13 T 09: 17: 05$ \\
14 & Spectrometer Frequency & 400.12 \\
15 & Spectral Width & 6402.0 \\
16 & Lowest Frequency & -809.0 \\
17 & Nucleus & $1 \mathrm{H}$ \\
18 & Acquired Size & 12824 \\
19 & Spectral Size & 65536 \\
\hline
\end{tabular}

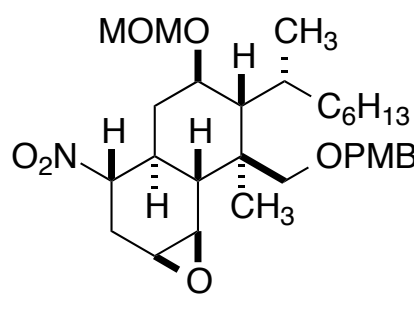

Compound 5

[Scheme 1, Step 1]

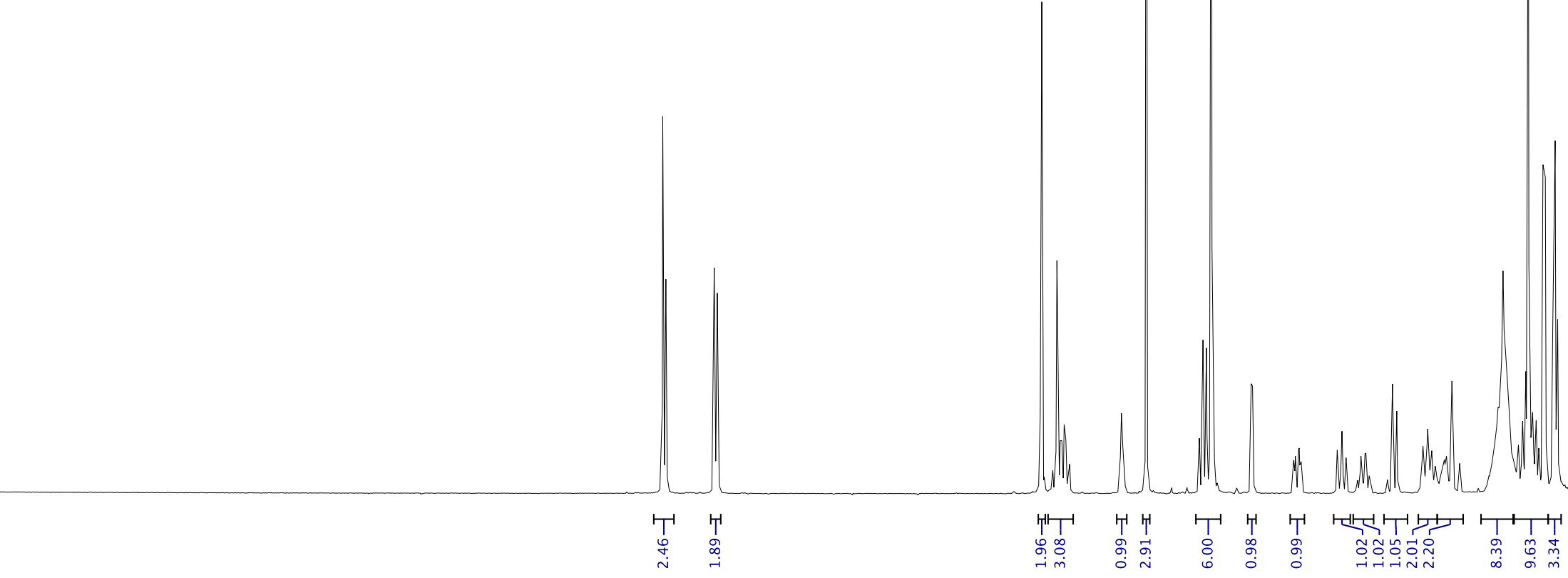




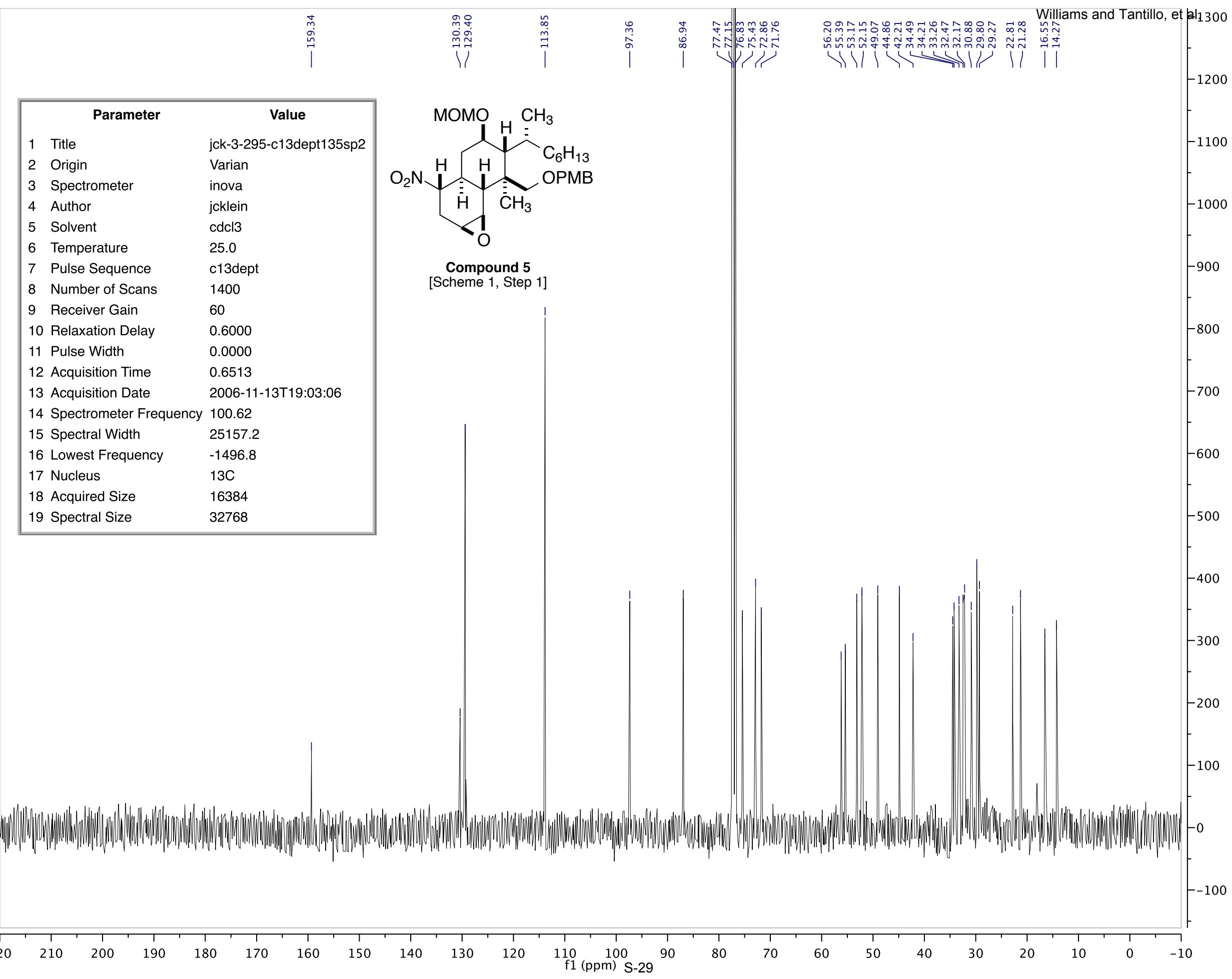




\begin{tabular}{|lll|}
\hline \multicolumn{1}{|c|}{ Parameter } & \multicolumn{1}{c|}{ Value } \\
1 & Title & jck-4-207 \\
2 & Origin & Varian \\
3 & Spectrometer & inova \\
4 & Author & jcklein \\
5 & Solvent & cdcl3 \\
6 & Temperature & 25.0 \\
7 & Pulse Sequence & s2pul \\
8 & Number of Scans & 8 \\
9 & Receiver Gain & 30 \\
10 & Relaxation Delay & 1.0000 \\
11 & Pulse Width & 0.0000 \\
12 & Acquisition Time & 2.7304 \\
13 & Acquisition Date & $2008-02-15 T 15: 59: 38$ \\
14 & Spectrometer Frequency & 399.71 \\
15 & Spectral Width & 6000.6 \\
16 & Lowest Frequency & -993.2 \\
17 & Nucleus & $1 \mathrm{H}$ \\
18 & Acquired Size & 16384 \\
19 & Spectral Size & 65536 \\
\hline
\end{tabular}

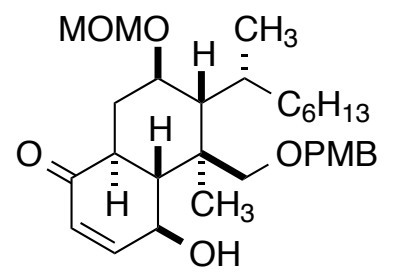

Compound 6

[Scheme 1, Step 2] 


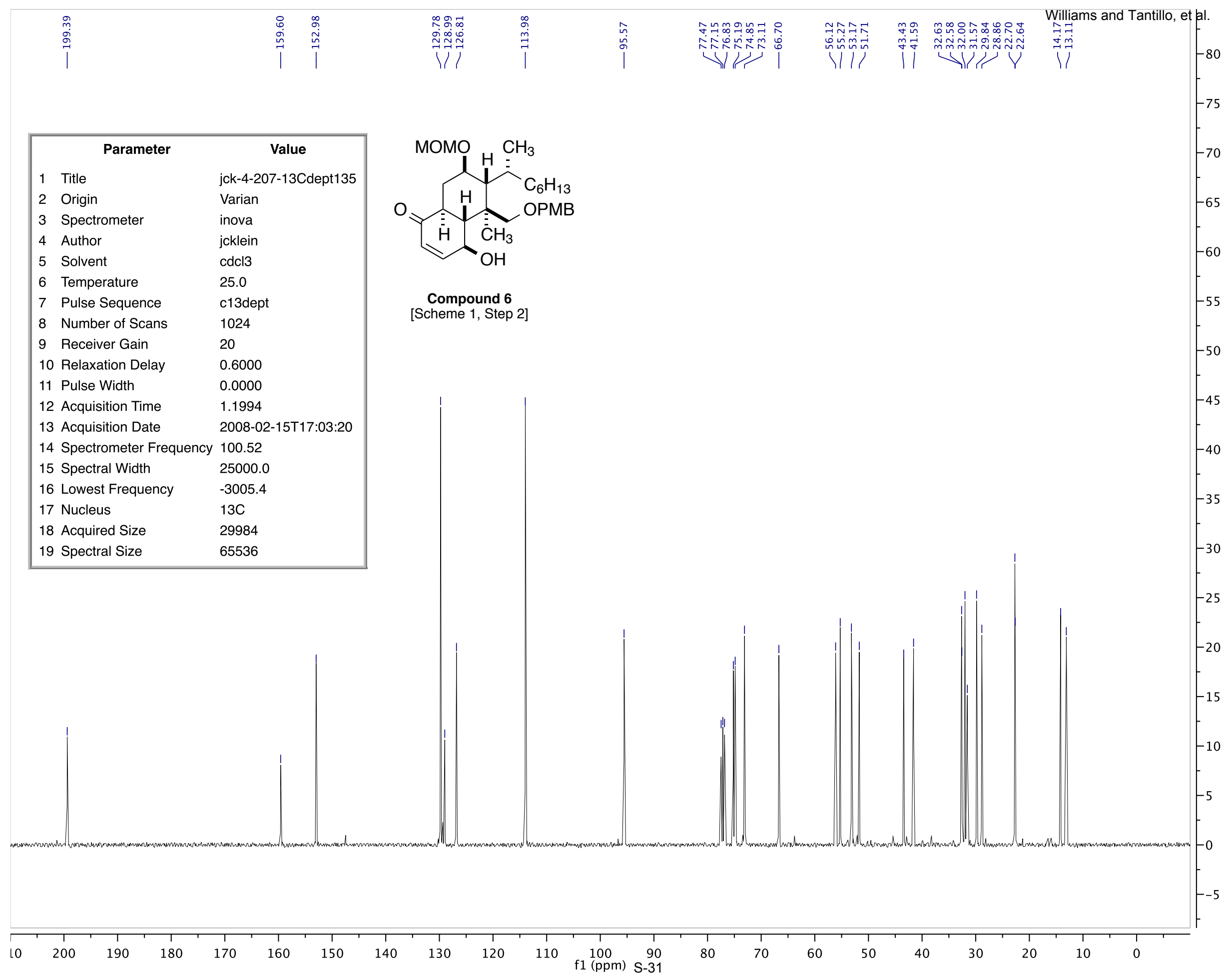




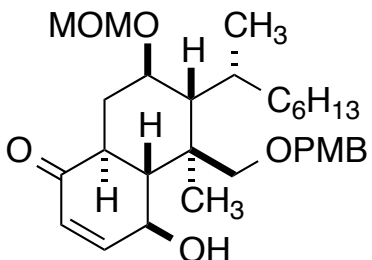

Compound 6 [Scheme 1, Step 2]

\begin{tabular}{|lll|}
\hline \multicolumn{1}{|c|}{ Parameter } & \multicolumn{1}{c|}{ Value } \\
1 & Title & jck-4-207-2dgdqcosy \\
2 & Origin & Varian \\
3 & Spectrometer & inova \\
4 & Author & jcklein \\
5 & Solvent & d2o \\
6 & Temperature & 25.0 \\
7 & Pulse Sequence & iugdqcosy \\
8 & Number of Scans & 4 \\
9 & Receiver Gain & 20 \\
10 & Relaxation Delay & 1.0000 \\
11 & Pulse Width & 670.0000 \\
12 & Acquisition Time & 0.1277 \\
13 & Acquisition Date & $2008-02-15 T 01: 48: 51$ \\
14 & Spectrometer Frequency & $(499.81,499.81)$ \\
15 & Spectral Width & $(4009.8,4009.8)$ \\
16 & Lowest Frequency & $(132.7,132.7)$ \\
17 & Nucleus & $(1 \mathrm{H}, 1 \mathrm{H})$ \\
18 & Acquired Size & $(512,200)$ \\
19 & Spectral Size & $(512,512)$ \\
\hline
\end{tabular}

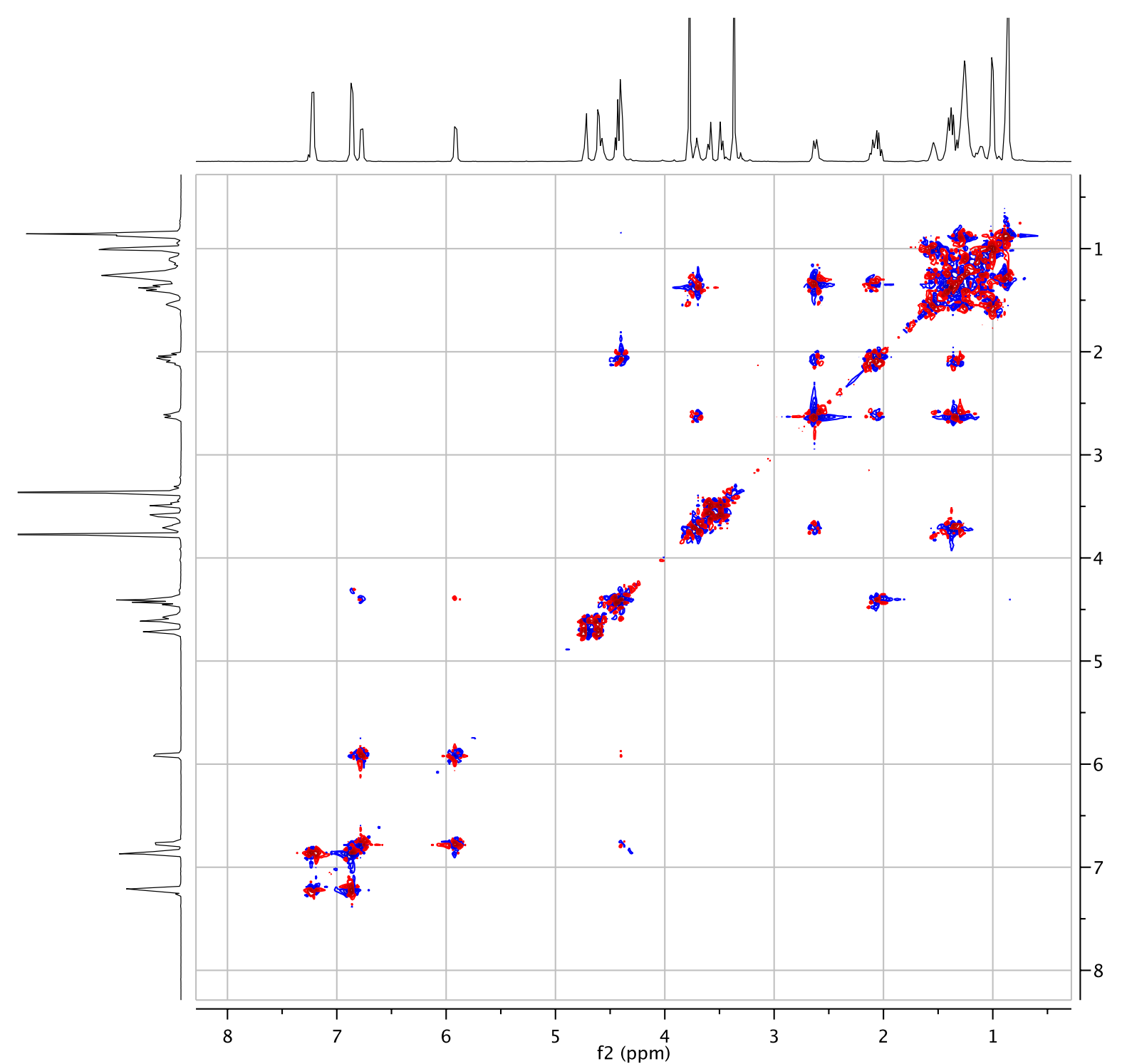




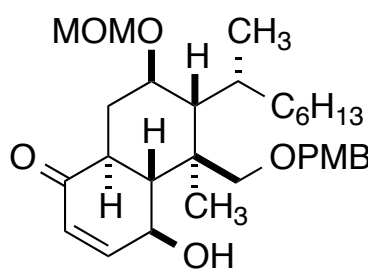

Compound 6

[Scheme 1, Step 2]

\begin{tabular}{|lll|}
\hline \multicolumn{1}{|c|}{ Parameter } & \multicolumn{1}{c|}{ Value } \\
1 & Title & jck-4-207-noesy \\
2 & Origin & Varian \\
3 & Spectrometer & inova \\
4 & Author & jcklein \\
5 & Solvent & d2o \\
6 & Temperature & 25.0 \\
7 & Pulse Sequence & iunoesy \\
8 & Number of Scans & 4 \\
9 & Receiver Gain & 20 \\
10 & Relaxation Delay & 1.5000 \\
11 & Pulse Width & 0.0000 \\
12 & Acquisition Time & 0.2554 \\
13 & Acquisition Date & $2008-02-15 T 02: 58: 38$ \\
14 & Spectrometer Frequency & $(499.81,499.81)$ \\
15 & Spectral Width & $(4009.8,4009.8)$ \\
16 & Lowest Frequency & $(132.7,132.7)$ \\
17 & Nucleus & $(1 \mathrm{H}, 1 \mathrm{H})$ \\
18 & Acquired Size & $(1024,200)$ \\
19 & Spectral Size & $(1024,1024)$ \\
\hline
\end{tabular}

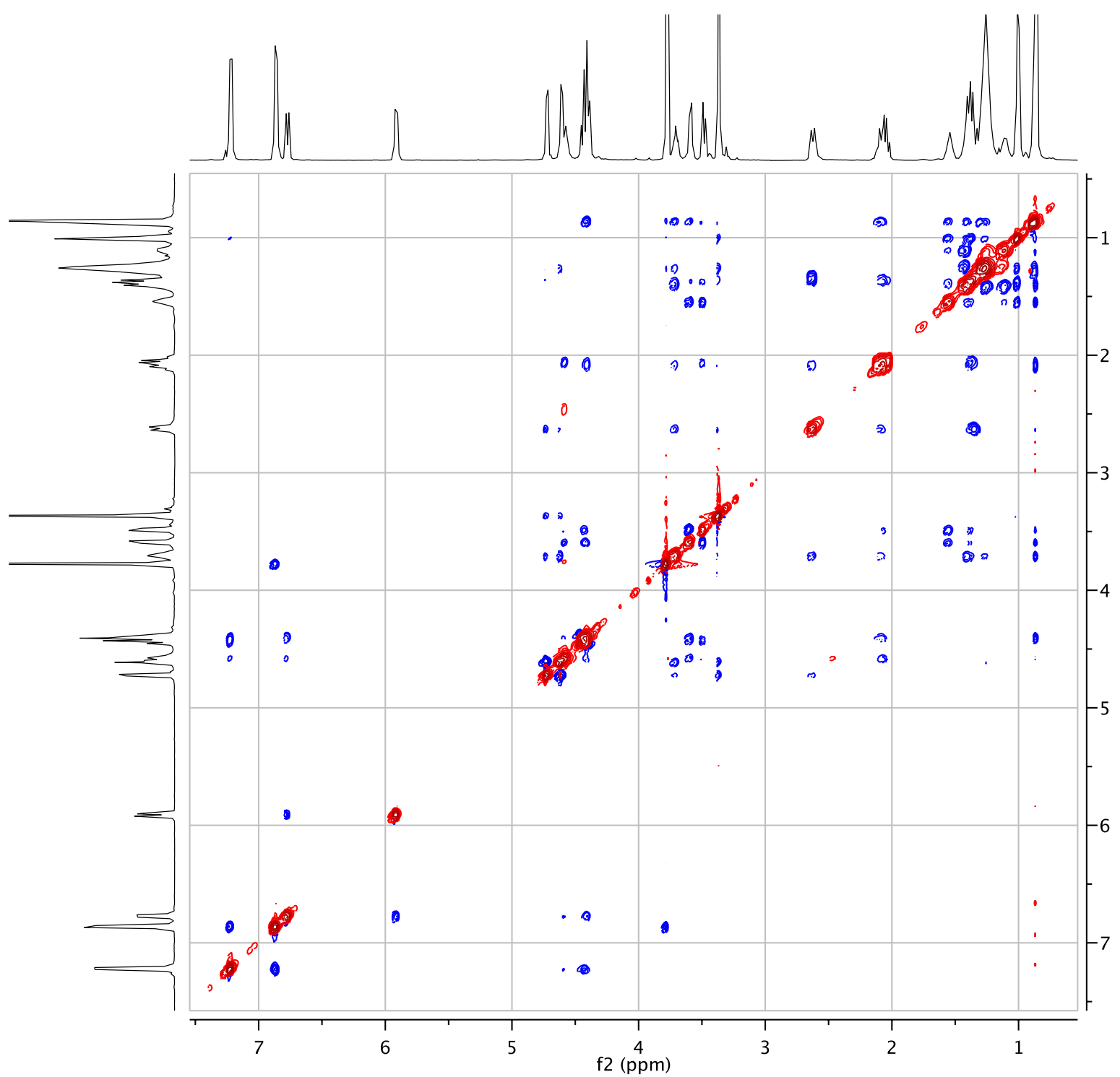

$\overrightarrow{\underline{\underline{2}}}$ 


\begin{tabular}{|lll|}
\hline \multicolumn{1}{|c|}{ Parameter } & \multicolumn{1}{c|}{ Value } \\
1 & Title & jck-3-117-1H \\
2 & Origin & Varian \\
3 & Spectrometer & inova \\
4 & Author & jcklein \\
5 & Solvent & cdcl3 \\
6 & Temperature & 25.0 \\
7 & Pulse Sequence & s2pul \\
8 & Number of Scans & 16 \\
9 & Receiver Gain & 20 \\
10 & Relaxation Delay & 1.0000 \\
11 & Pulse Width & 0.0000 \\
12 & Acquisition Time & 2.0029 \\
13 & Acquisition Date & $2005-09-24 T 23: 08: 46$ \\
14 & Spectrometer Frequency & 400.12 \\
15 & Spectral Width & 3019.6 \\
16 & Lowest Frequency & 11.8 \\
17 & Nucleus & $1 \mathrm{H}$ \\
18 & Acquired Size & 6048 \\
19 & Spectral Size & 65536 \\
\hline
\end{tabular}

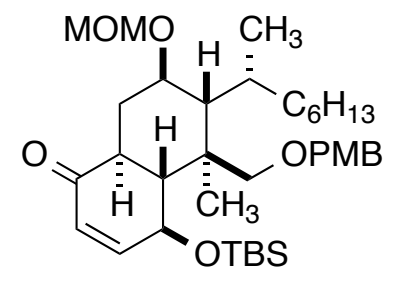

Compound 6a

[Scheme 1, Step 6]

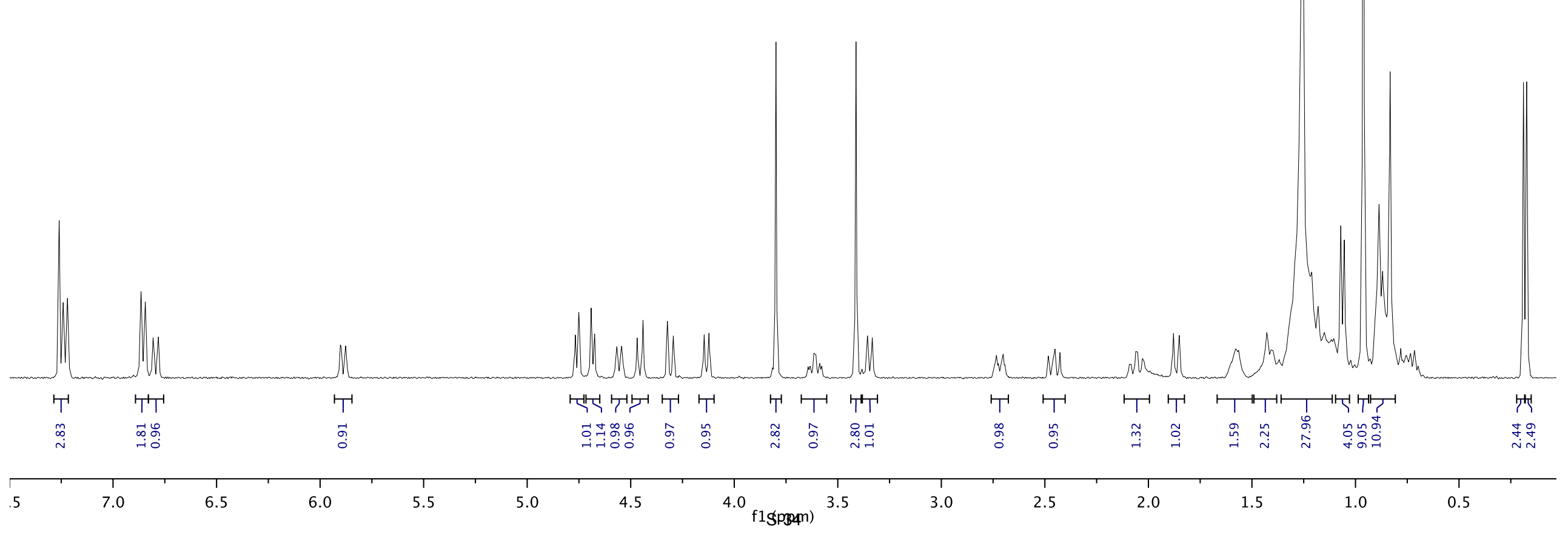




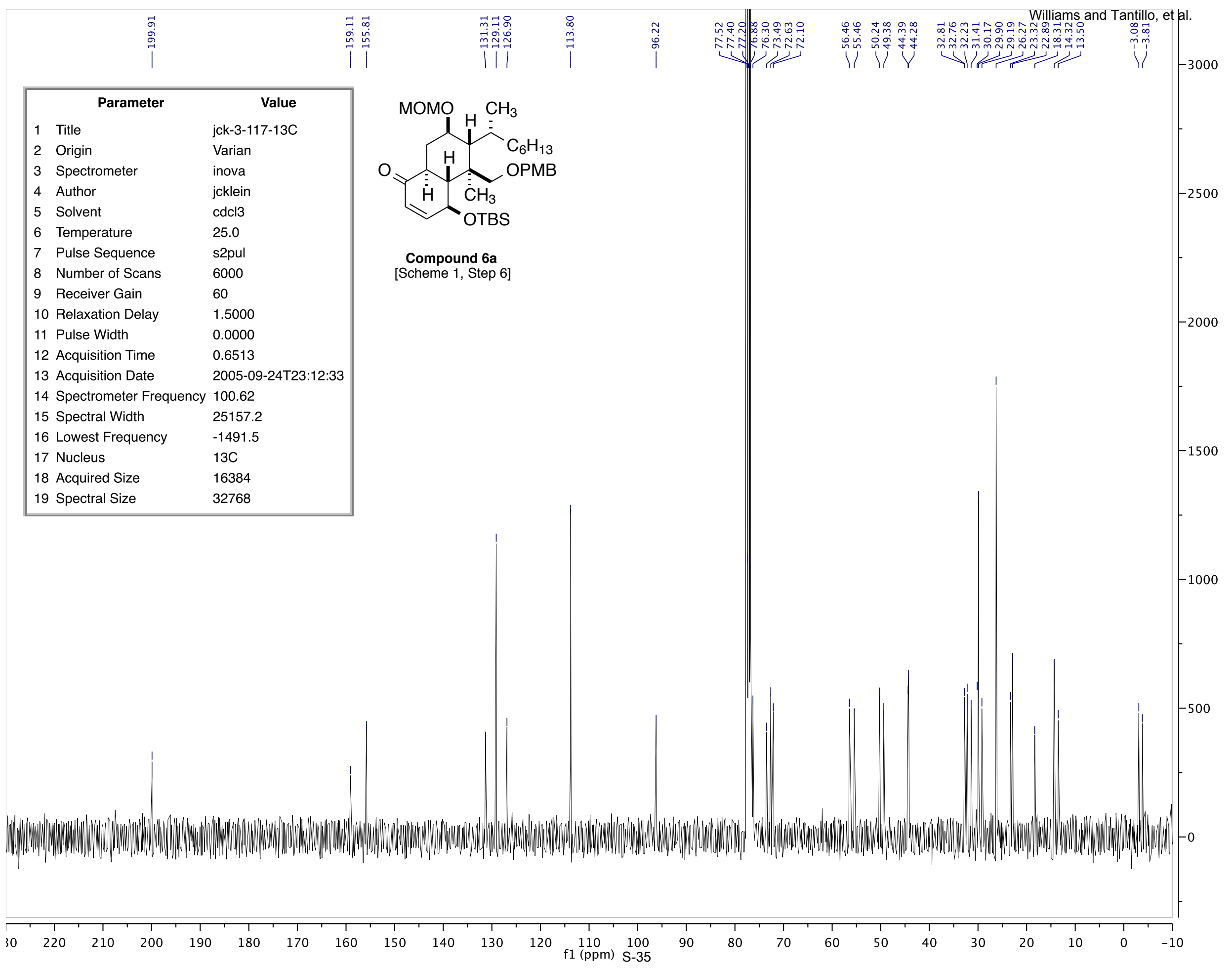




\begin{tabular}{|lll|}
\hline \multicolumn{1}{|c|}{ Parameter } & \multicolumn{1}{c|}{ Value } \\
1 & Title & jck-4-86 \\
2 & Origin & Varian \\
3 & Spectrometer & inova \\
4 & Author & jcklein \\
5 & Solvent & cdcl3 \\
6 & Temperature & 25.0 \\
7 & Pulse Sequence & s2pul \\
8 & Number of Scans & 16 \\
9 & Receiver Gain & 36 \\
10 & Relaxation Delay & 1.0000 \\
11 & Pulse Width & 0.0000 \\
12 & Acquisition Time & 2.0031 \\
13 & Acquisition Date & $2007-03-20 \mathrm{~T} 18: 03: 47$ \\
14 & Spectrometer Frequency & 400.12 \\
15 & Spectral Width & 6402.0 \\
16 & Lowest Frequency & -810.0 \\
17 & Nucleus & $1 \mathrm{H}$ \\
18 & Acquired Size & 12824 \\
19 & Spectral Size & 65536 \\
\hline
\end{tabular}

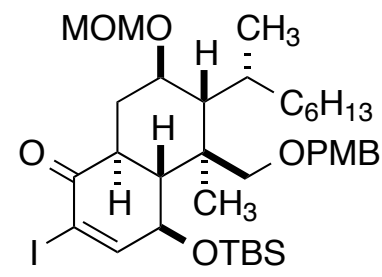

Compound 7

[Scheme 1, Step 4]

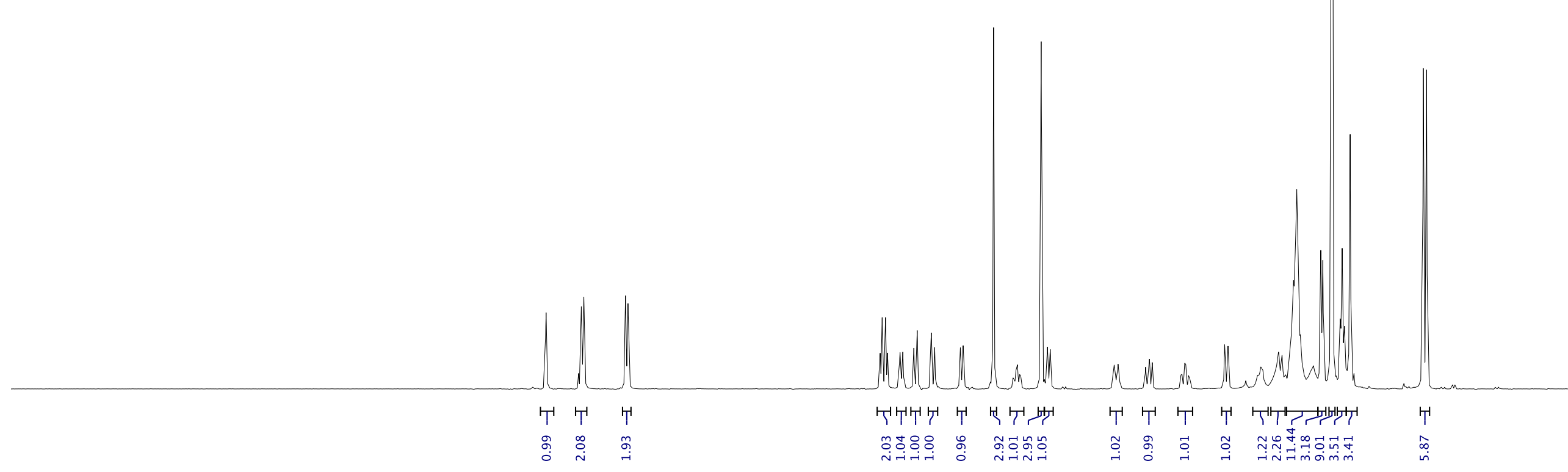

6.5

$\begin{array}{lcc}6.0 & 5.5 & 5.0\end{array}$

4.5




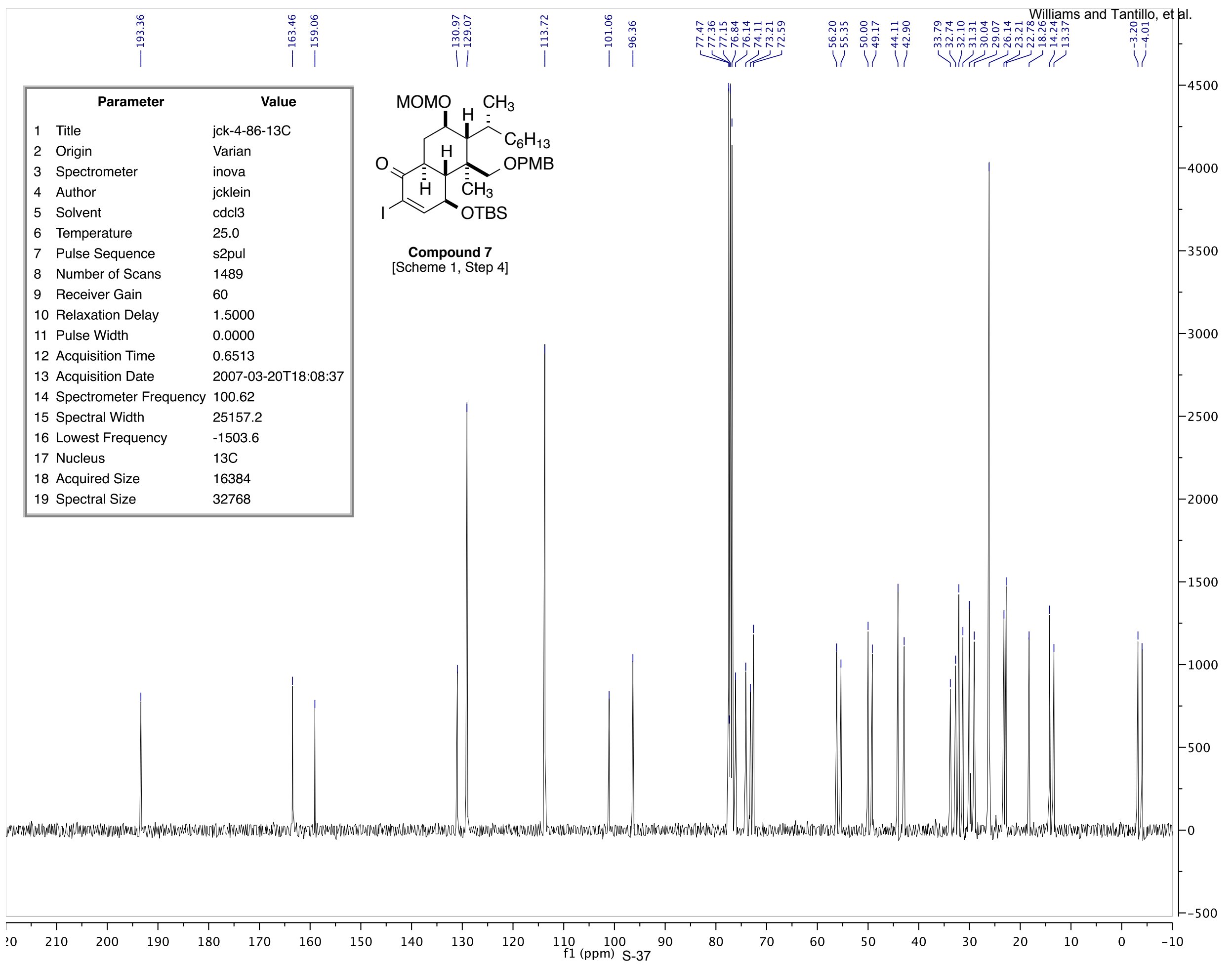




\begin{tabular}{|lll|}
\hline \multicolumn{1}{|c|}{ Parameter } & \multicolumn{1}{c|}{ Value } \\
1 & Title & jck-4-69 \\
2 & Origin & Varian \\
3 & Spectrometer & inova \\
4 & Author & jcklein \\
5 & Solvent & cdcl3 \\
6 & Temperature & 25.0 \\
7 & Pulse Sequence & s2pul \\
8 & Number of Scans & 32 \\
9 & Receiver Gain & 48 \\
10 & Relaxation Delay & 1.0000 \\
11 & Pulse Width & 0.0000 \\
12 & Acquisition Time & 2.0031 \\
13 & Acquisition Date & $2007-02-13 T 11: 08: 00$ \\
14 & Spectrometer Frequency & 400.12 \\
15 & Spectral Width & 6402.0 \\
16 & Lowest Frequency & -810.4 \\
17 & Nucleus & $1 \mathrm{H}$ \\
18 & Acquired Size & 12824 \\
19 & Spectral Size & 65536 \\
\hline
\end{tabular}

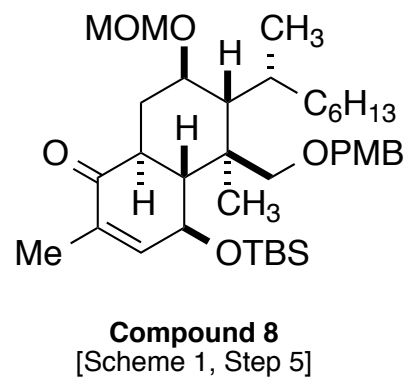

[Scheme 1, Step 5]

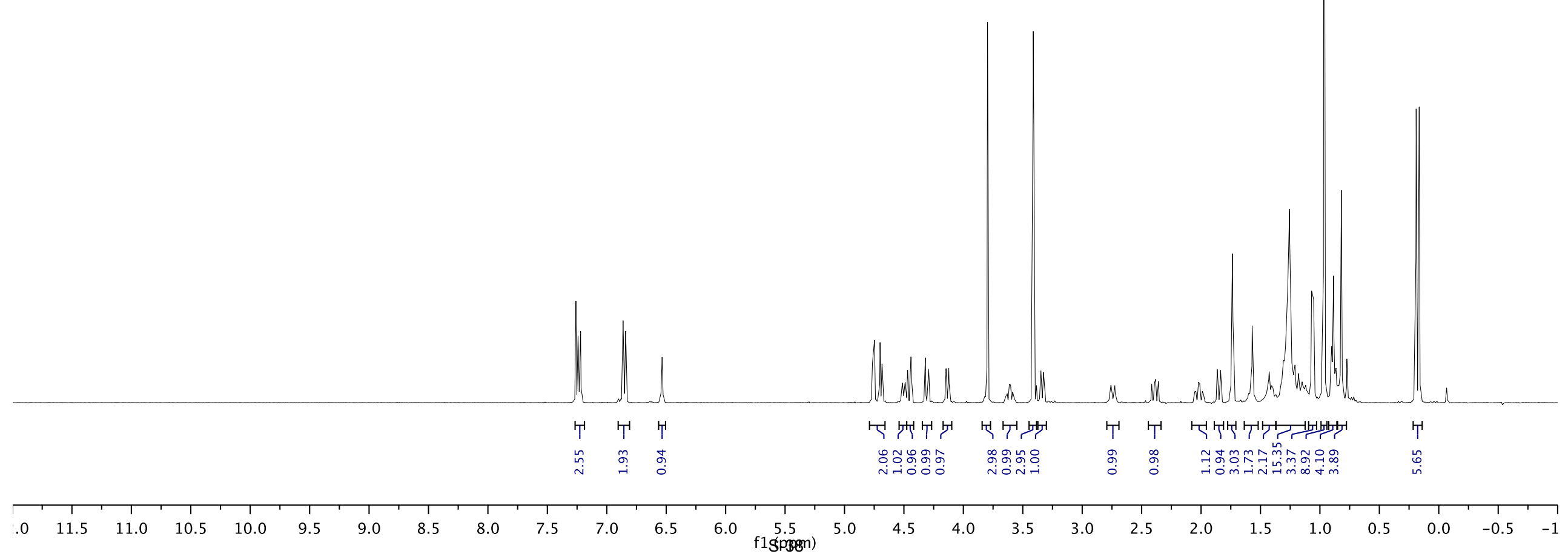




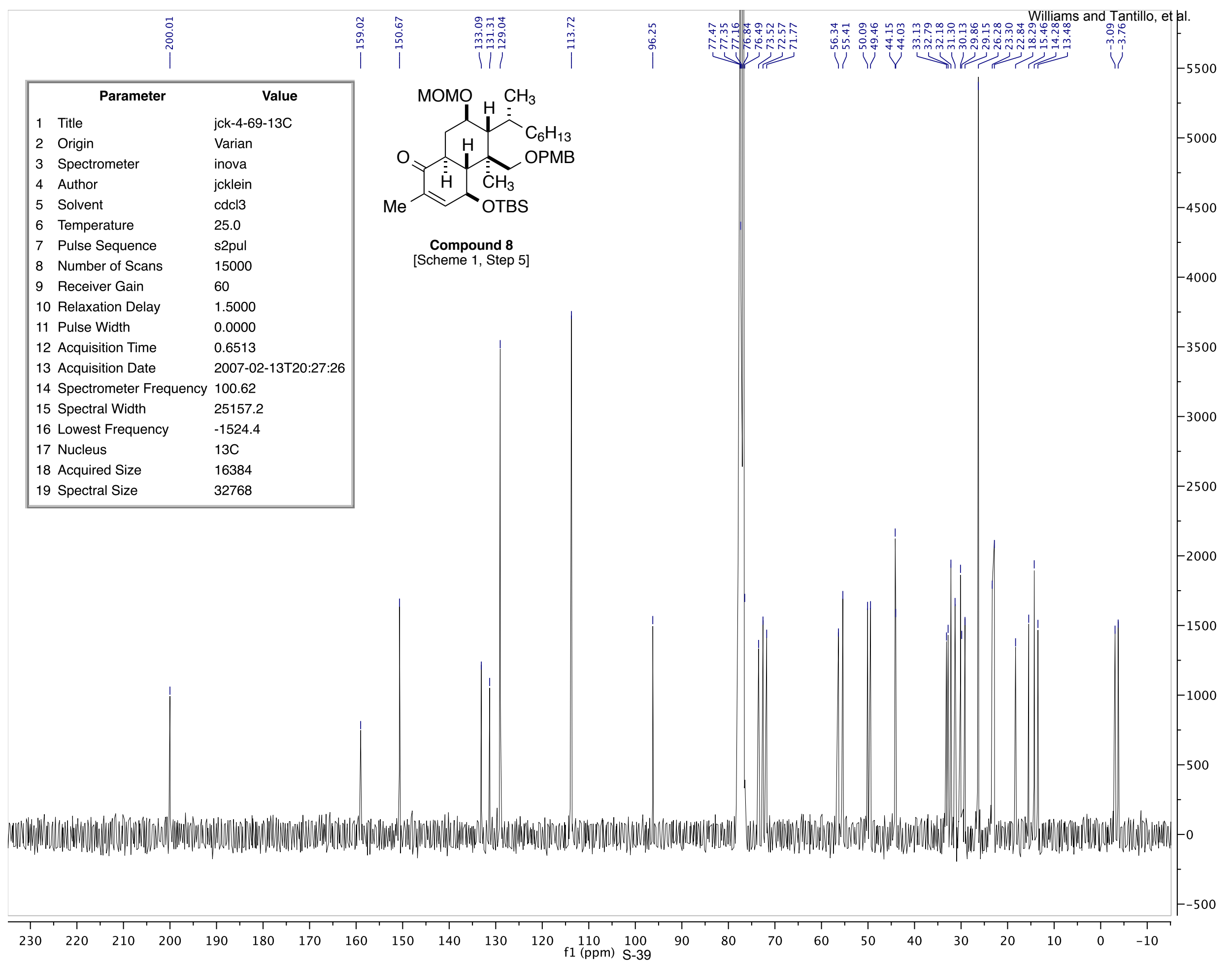




\begin{tabular}{|lll|}
\hline \multicolumn{1}{|c|}{ Parameter } & \multicolumn{1}{c|}{ Value } \\
1 & Title & jck-4-89 \\
2 & Origin & Varian \\
3 & Spectrometer & inova \\
4 & Author & jcklein \\
5 & Solvent & cdcl3 \\
6 & Temperature & 25.0 \\
7 & Pulse Sequence & s2pul \\
8 & Number of Scans & 16 \\
9 & Receiver Gain & 20 \\
10 & Relaxation Delay & 1.0000 \\
11 & Pulse Width & 0.0000 \\
12 & Acquisition Time & 2.0032 \\
13 & Acquisition Date & $2007-04-06 \mathrm{~T} 21: 15: 02$ \\
14 & Spectrometer Frequency & 400.12 \\
15 & Spectral Width & 3507.4 \\
16 & Lowest Frequency & -319.6 \\
17 & Nucleus & $1 \mathrm{H}$ \\
18 & Acquired Size & 7026 \\
19 & Spectral Size & 65536 \\
\hline
\end{tabular}

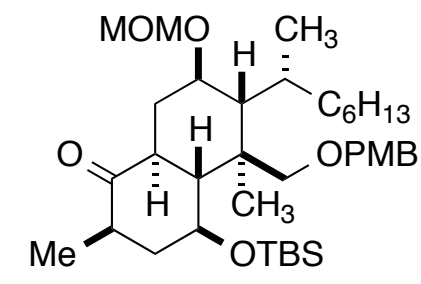

Compound 9

[Scheme 1, Step 6]

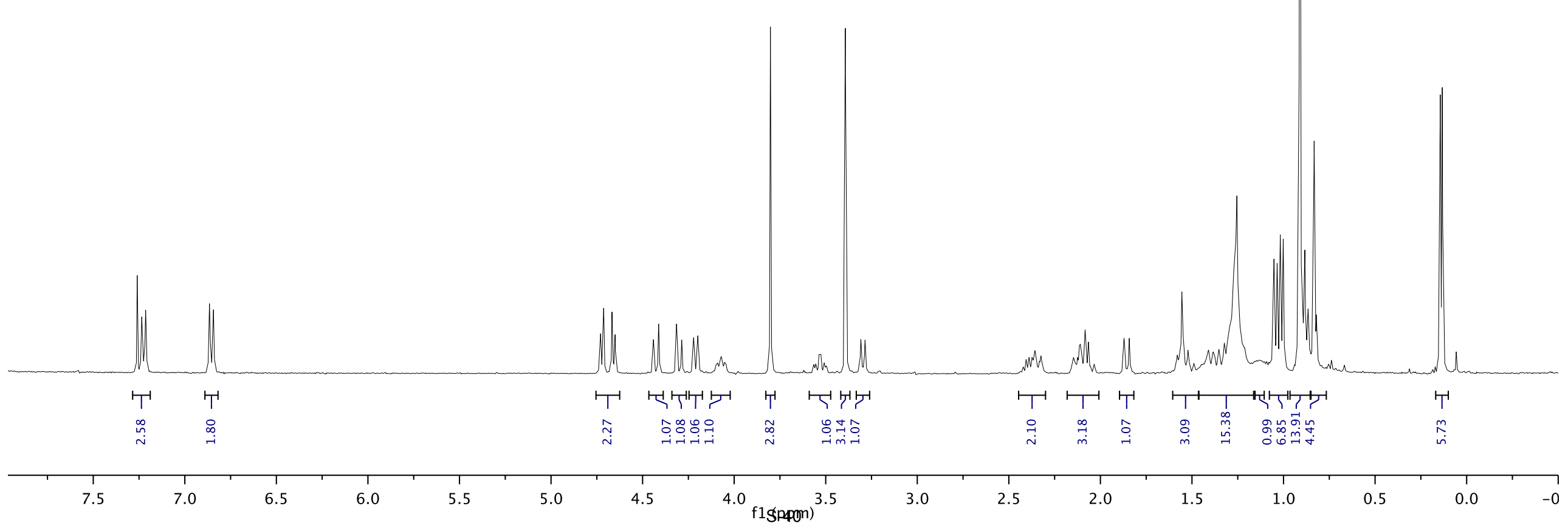




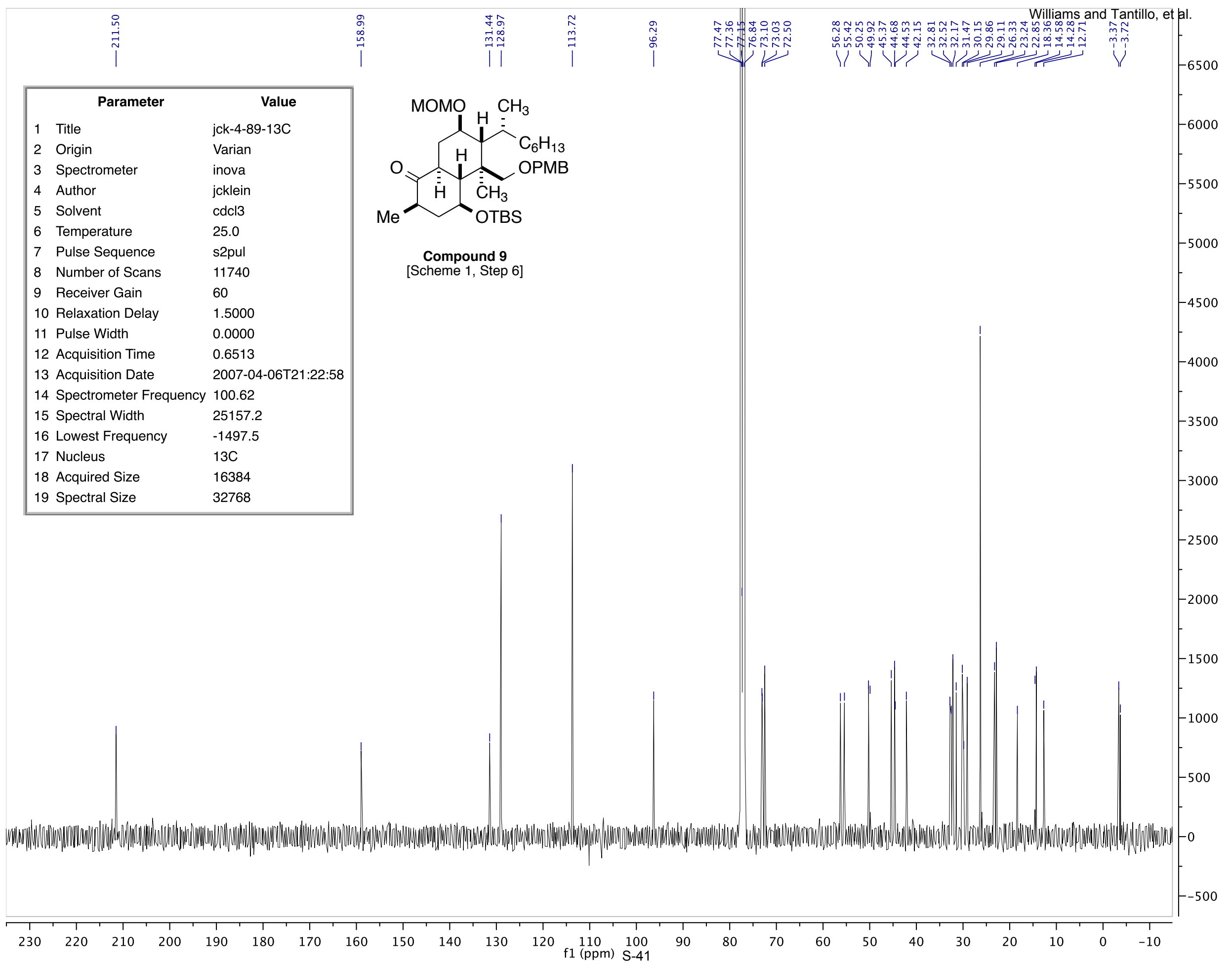




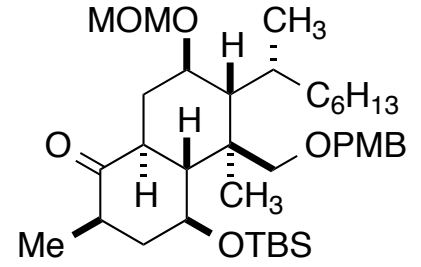

Compound 9

[Scheme 1, Step 6]

\begin{tabular}{|lll|}
\hline \multicolumn{1}{|c|}{ Parameter } & \multicolumn{1}{c|}{ Value } \\
1 & Title & jck-4-89-2dgdqcosy \\
2 & Origin & Varian \\
3 & Spectrometer & inova \\
4 & Author & jcklein \\
5 & Solvent & cdcl3 \\
6 & Temperature & 25.0 \\
7 & Pulse Sequence & gDQCOSY \\
8 & Number of Scans & 8 \\
9 & Receiver Gain & 60 \\
10 & Relaxation Delay & 1.0000 \\
11 & Pulse Width & 0.0000 \\
12 & Acquisition Time & 0.1460 \\
13 & Acquisition Date & $2007-04-07 T 01: 48: 46$ \\
14 & Spectrometer Frequency & $(400.12,400.12)$ \\
15 & Spectral Width & $(3507.4,3507.4)$ \\
16 & Lowest Frequency & $(-319.6,-319.6)$ \\
17 & Nucleus & $(1 \mathrm{H}, 1 \mathrm{H})$ \\
18 & Acquired Size & $(512,200)$ \\
19 & Spectral Size & $(512,512)$ \\
\hline
\end{tabular}

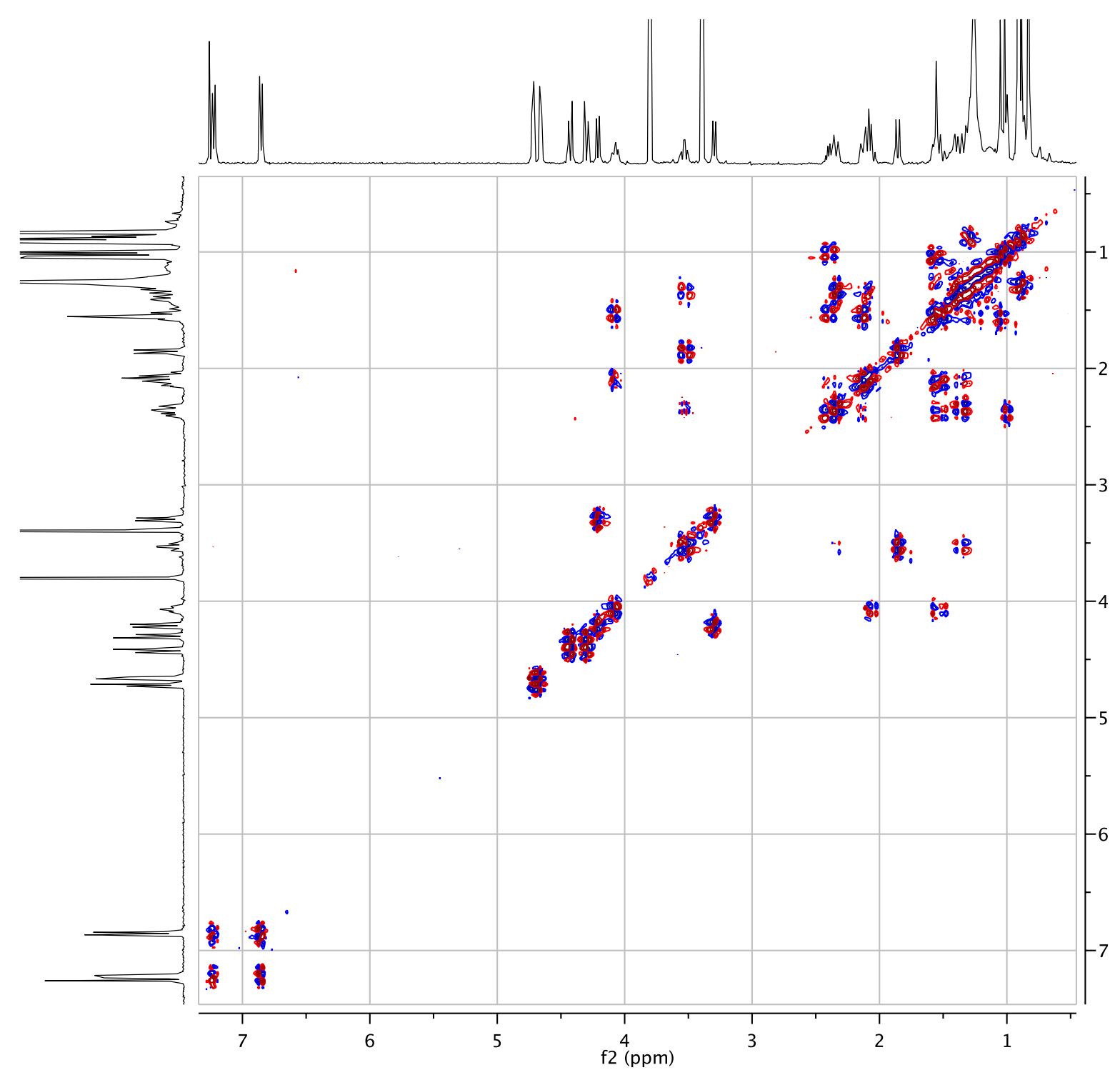




\begin{tabular}{|lll|}
\hline \multicolumn{1}{|c|}{ Parameter } & \multicolumn{1}{c|}{ Value } \\
1 & Title & jck-4-204-B \\
2 & Origin & Varian \\
3 & Spectrometer & inova \\
4 & Author & jcklein \\
5 & Solvent & cdcl3 \\
6 & Temperature & 25.0 \\
7 & Pulse Sequence & s2pul \\
8 & Number of Scans & 16 \\
9 & Receiver Gain & 52 \\
10 & Relaxation Delay & 1.0000 \\
11 & Pulse Width & 0.0000 \\
12 & Acquisition Time & 2.7304 \\
13 & Acquisition Date & $2008-02-18 T 11: 36: 43$ \\
14 & Spectrometer Frequency & 399.71 \\
15 & Spectral Width & 6000.6 \\
16 & Lowest Frequency & -993.5 \\
17 & Nucleus & $1 \mathrm{H}$ \\
18 & Acquired Size & 16384 \\
19 & Spectral Size & 65536 \\
\hline
\end{tabular}

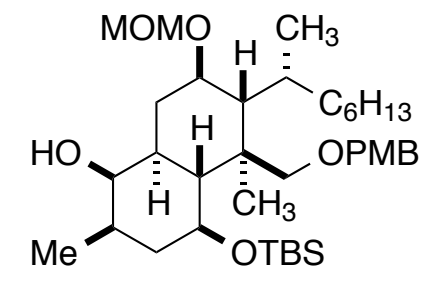

Compound 9a

[Scheme 1, Step 7]

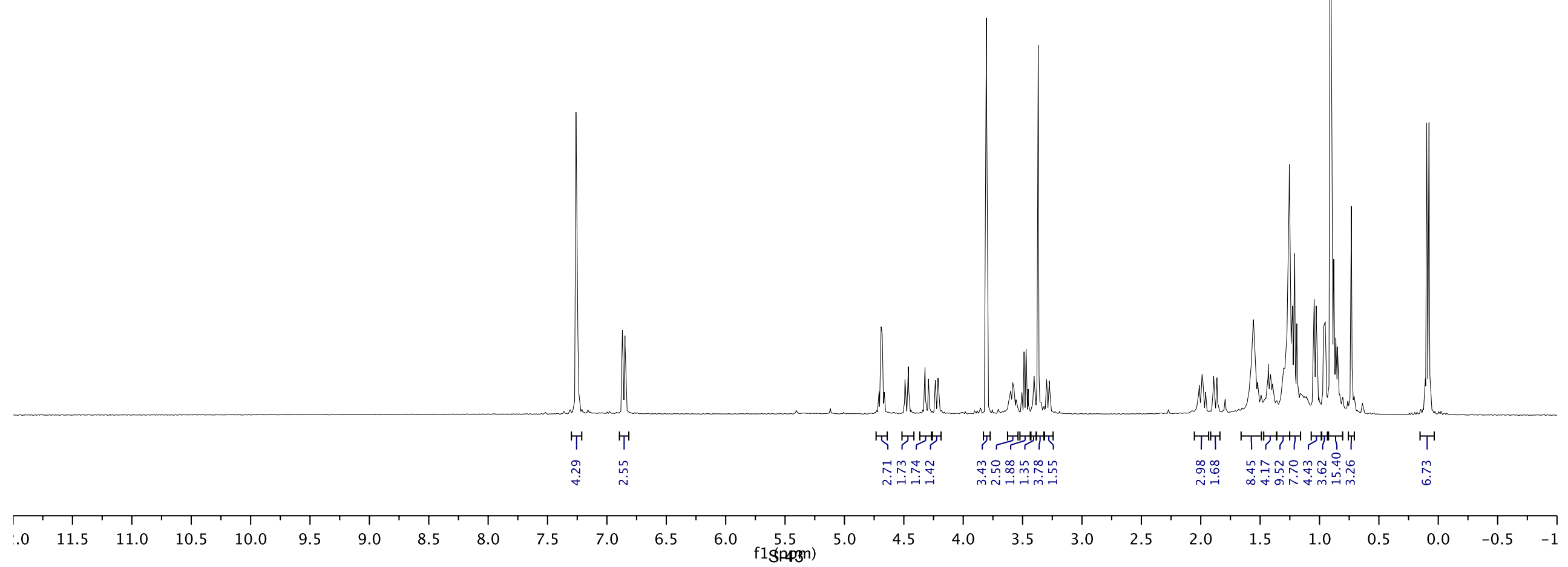




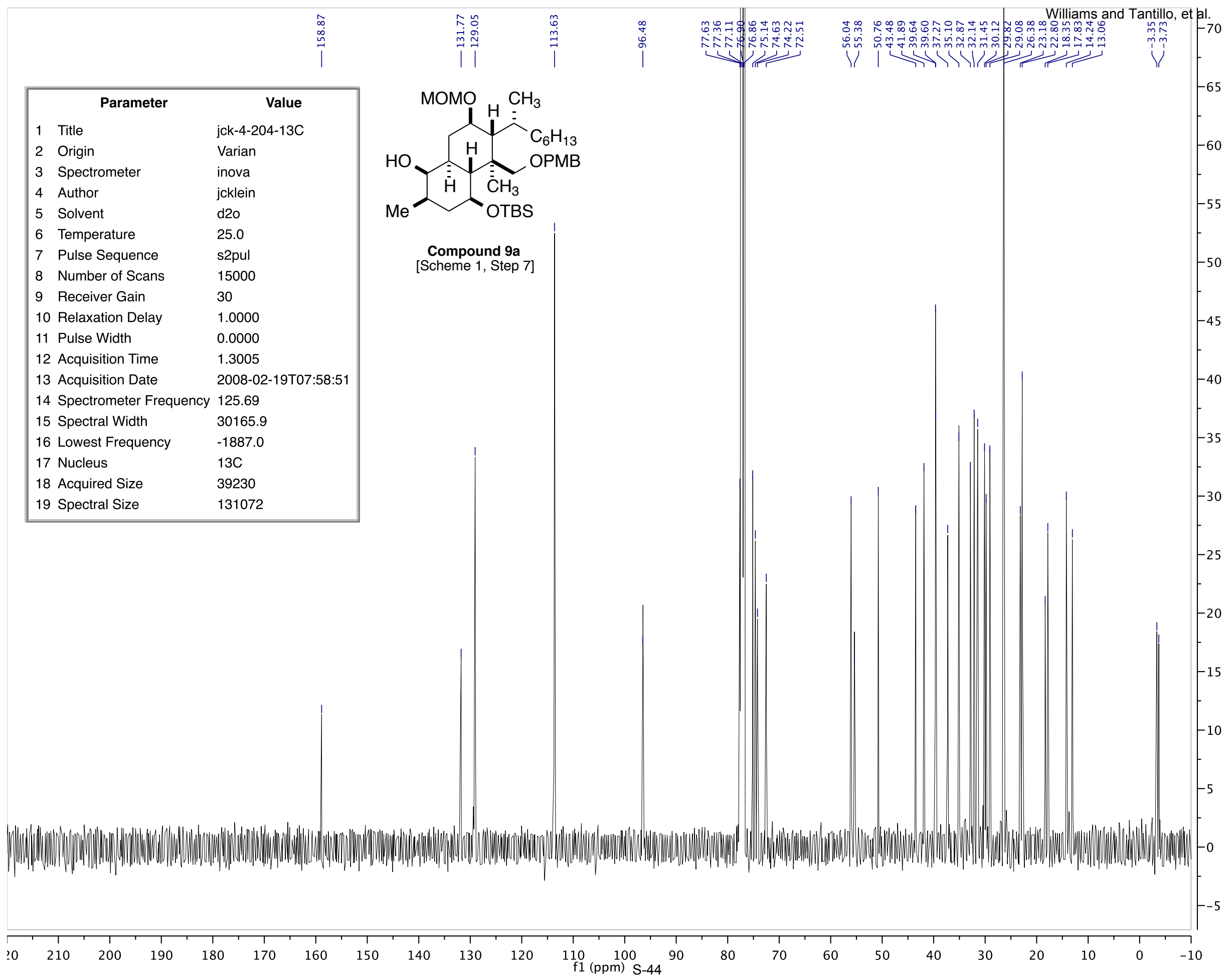




\begin{tabular}{|lll|}
\hline \multicolumn{1}{|c|}{ Parameter } & \multicolumn{1}{c|}{ Value } \\
1 & Title & jck-4-227-A \\
2 & Origin & Varian \\
3 & Spectrometer & inova \\
4 & Author & jcklein \\
5 & Solvent & cdcl3 \\
6 & Temperature & 25.0 \\
7 & Pulse Sequence & s2pul \\
8 & Number of Scans & 4 \\
9 & Receiver Gain & 46 \\
10 & Relaxation Delay & 1.0000 \\
11 & Pulse Width & 0.0000 \\
12 & Acquisition Time & 2.7304 \\
13 & Acquisition Date & $2008-03-28 T 22: 02: 43$ \\
14 & Spectrometer Frequency & 399.71 \\
15 & Spectral Width & 6000.6 \\
16 & Lowest Frequency & -993.2 \\
17 & Nucleus & $1 \mathrm{H}$ \\
18 & Acquired Size & 16384 \\
19 & Spectral Size & 65536 \\
\hline
\end{tabular}
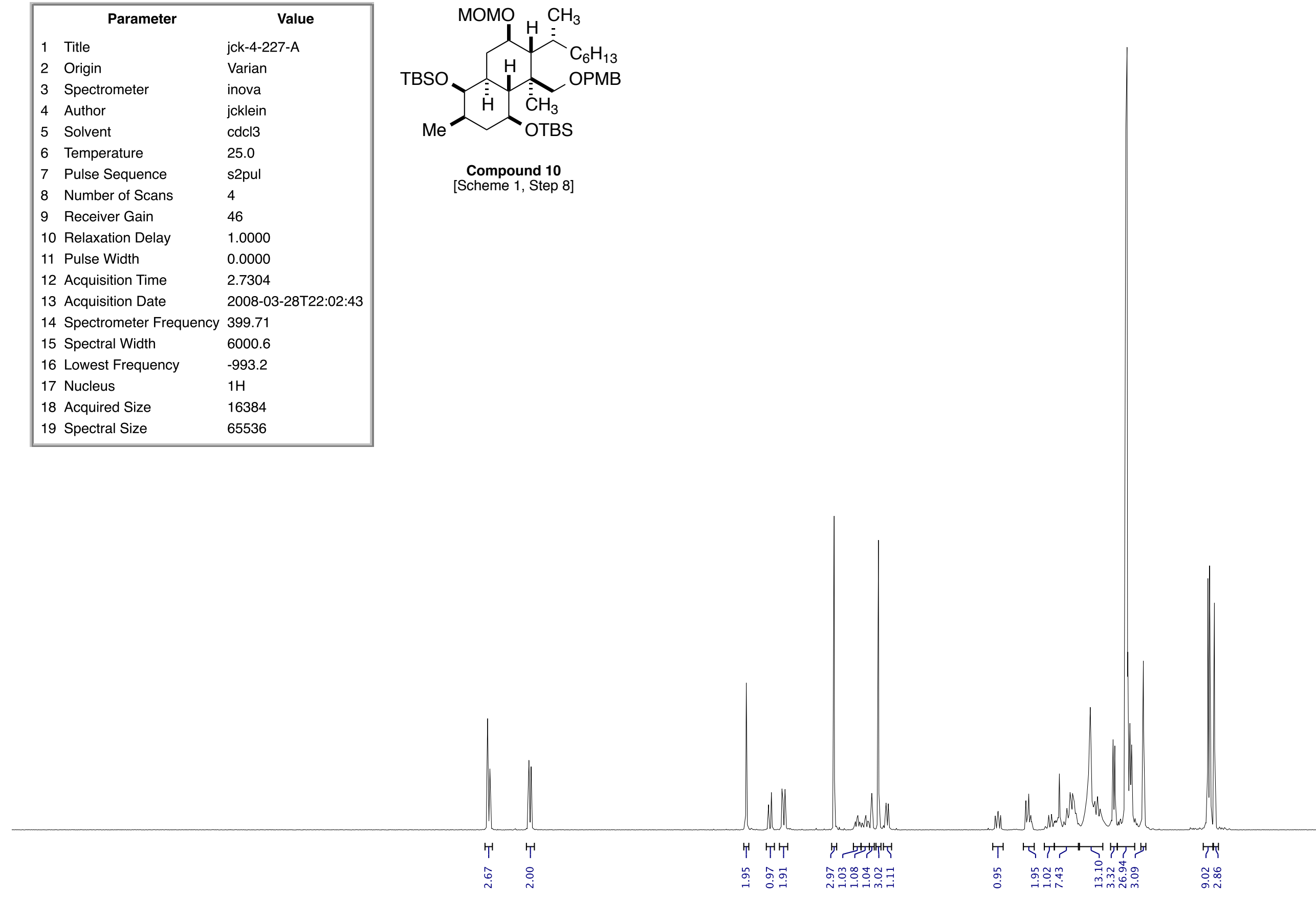

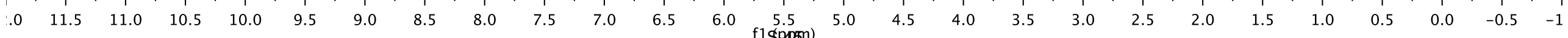




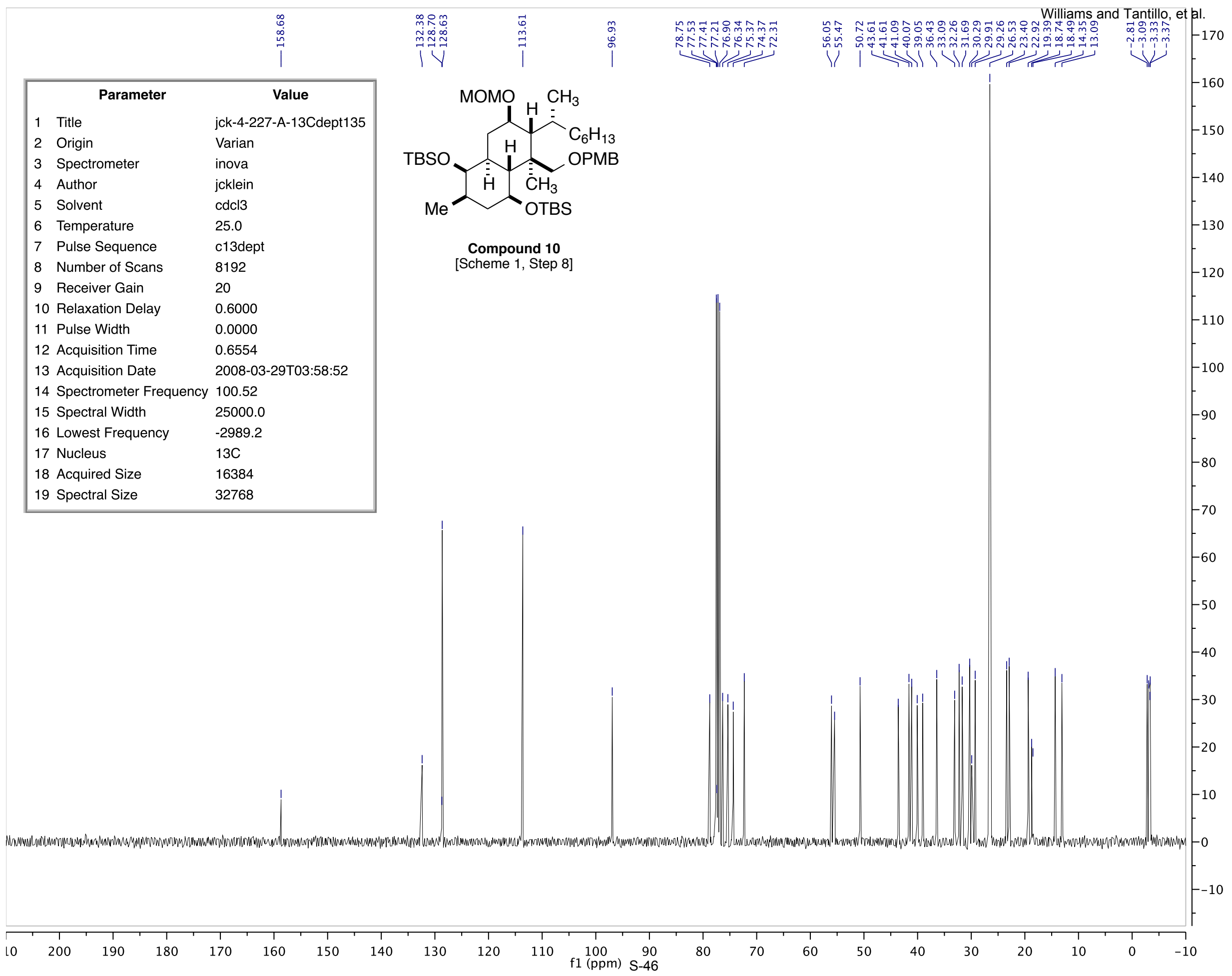




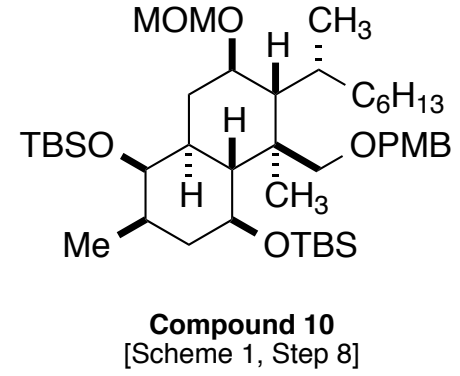

\begin{tabular}{|lll|}
\hline \multicolumn{1}{|c|}{ Parameter } & \multicolumn{1}{c|}{ Value } \\
1 & Title & jck-4-227-A-2dgdqcosy \\
2 & Origin & Varian \\
3 & Spectrometer & inova \\
4 & Author & jcklein \\
5 & Solvent & cdcl3 \\
6 & Temperature & 25.0 \\
7 & Pulse Sequence & gDQCOSY \\
8 & Number of Scans & 4 \\
9 & Receiver Gain & 48 \\
10 & Relaxation Delay & 1.0000 \\
11 & Pulse Width & 0.0000 \\
12 & Acquisition Time & 0.1571 \\
13 & Acquisition Date & $2008-03-31 T 22: 45: 36$ \\
14 & Spectrometer Frequency & $(400.12,400.12)$ \\
15 & Spectral Width & $(3258.1,3258.1)$ \\
16 & Lowest Frequency & $(-171.6,-171.6)$ \\
17 & Nucleus & $(1 \mathrm{H}, 1 \mathrm{H})$ \\
18 & Acquired Size & $(512,200)$ \\
19 & Spectral Size & $(512,512)$ \\
\hline
\end{tabular}

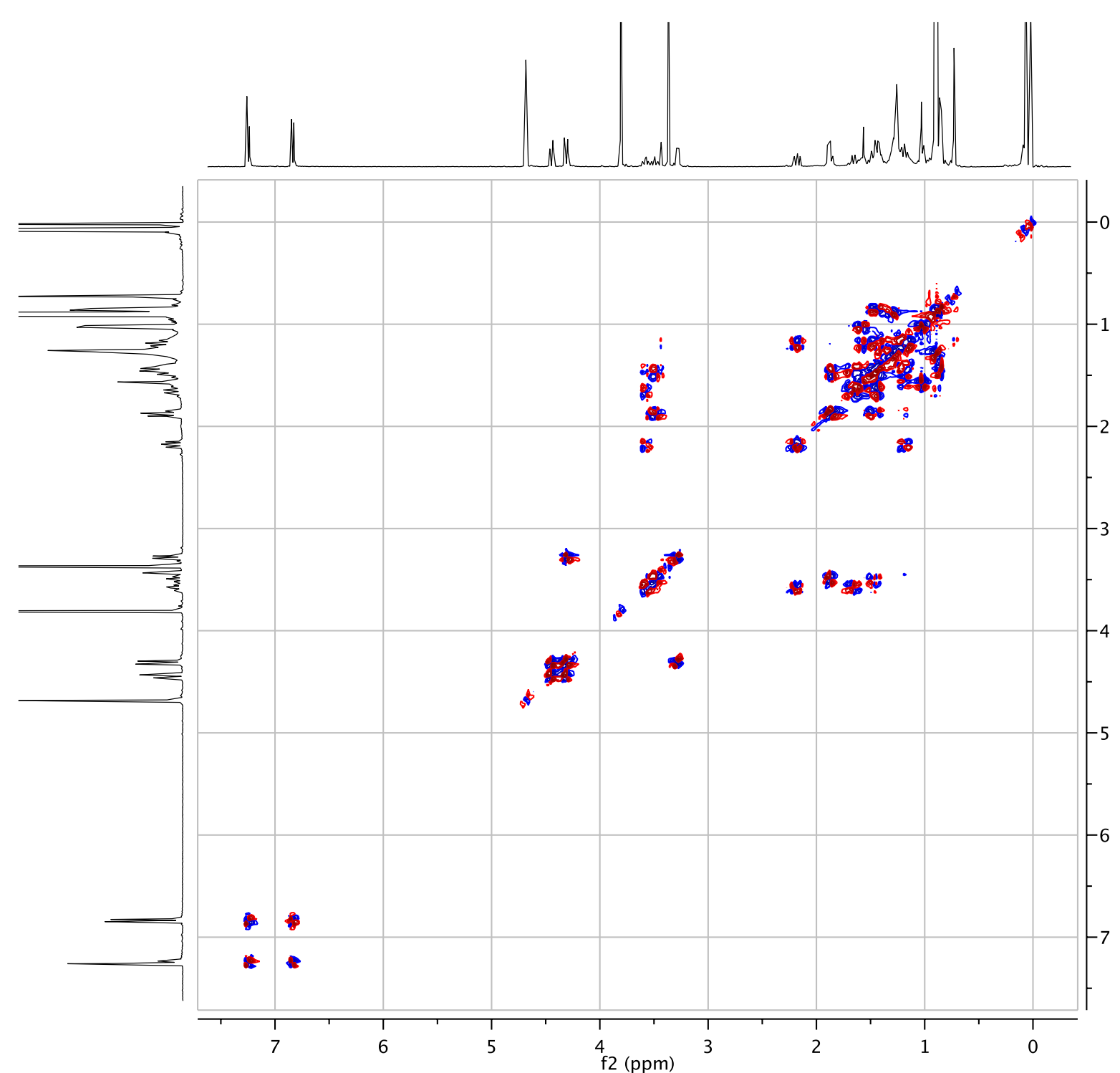

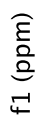




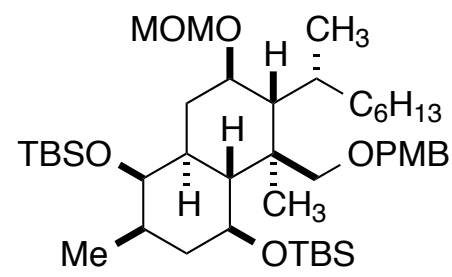

Compound 10

[Scheme 1, Step 8]

\begin{tabular}{|lll|}
\hline \multicolumn{1}{|c|}{ Parameter } & \multicolumn{1}{c|}{ Value } \\
1 & Title & jck-4-227-A-noesy \\
2 & Origin & Varian \\
3 & Spectrometer & inova \\
4 & Author & jcklein \\
5 & Solvent & cdcl3 \\
6 & Temperature & 25.0 \\
7 & Pulse Sequence & iunoesy \\
8 & Number of Scans & 4 \\
9 & Receiver Gain & 48 \\
10 & Relaxation Delay & 1.5000 \\
11 & Pulse Width & 0.0000 \\
12 & Acquisition Time & 0.3143 \\
13 & Acquisition Date & $2008-03-31 \mathrm{~T} 23: 57: 02$ \\
14 & Spectrometer Frequency & $(400.12,400.12)$ \\
15 & Spectral Width & $(3258.1,3258.1)$ \\
16 & Lowest Frequency & $(-171.6,-171.6)$ \\
17 & Nucleus & $(1 \mathrm{H}, 1 \mathrm{H})$ \\
18 & Acquired Size & $(1024,200)$ \\
19 & Spectral Size & $(1024,1024)$ \\
\hline
\end{tabular}

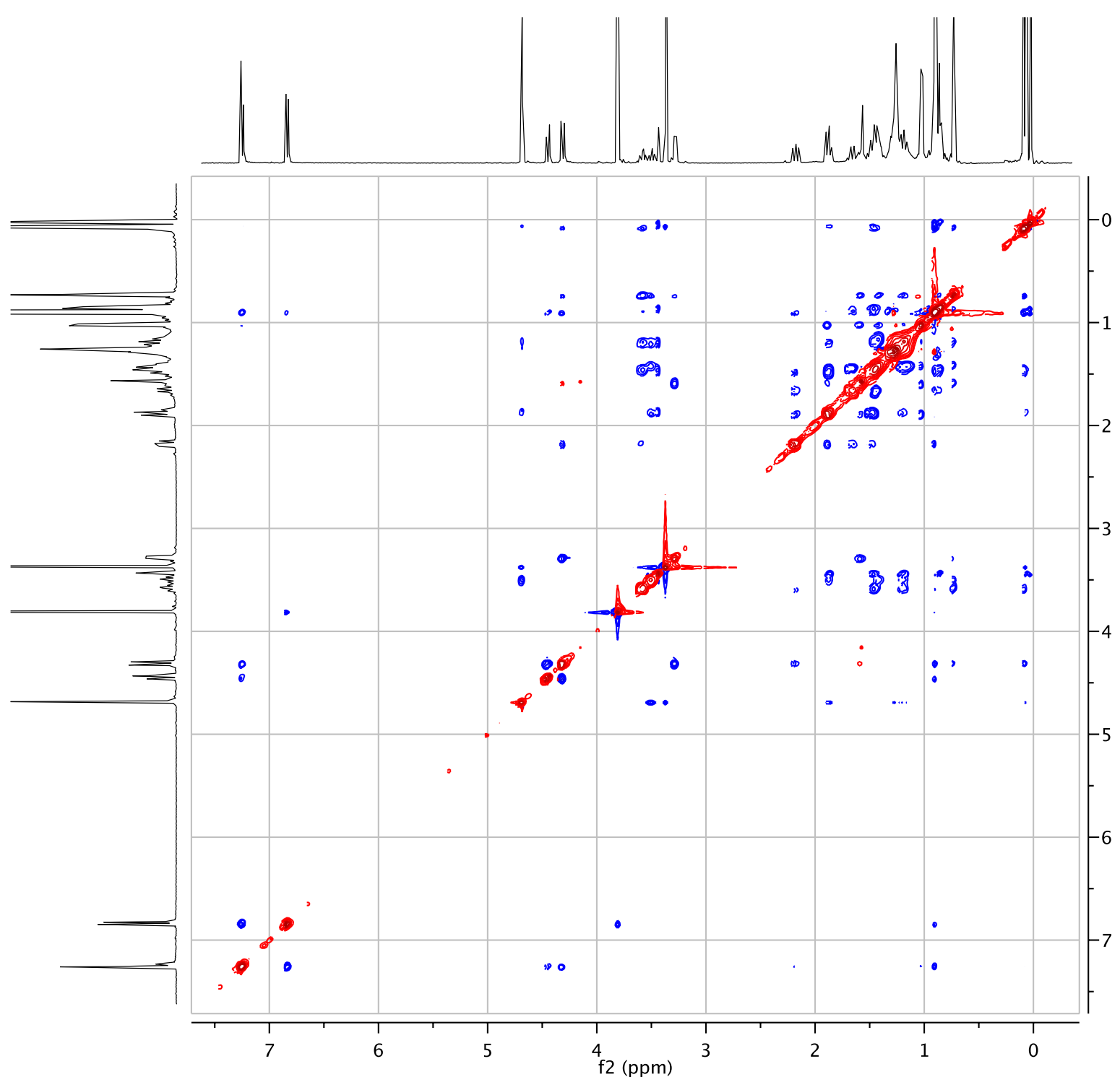

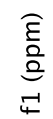




\begin{tabular}{|lll|}
\hline \multicolumn{1}{|c|}{ Parameter } & \multicolumn{1}{c|}{ Value } \\
1 & Title & jck-4-246-C \\
2 & Origin & Varian \\
3 & Spectrometer & inova \\
4 & Author & jcklein \\
5 & Solvent & cdcl3 \\
6 & Temperature & 25.0 \\
7 & Pulse Sequence & s2pul \\
8 & Number of Scans & 16 \\
9 & Receiver Gain & 58 \\
10 & Relaxation Delay & 1.0000 \\
11 & Pulse Width & 0.0000 \\
12 & Acquisition Time & 2.7304 \\
13 & Acquisition Date & $2008-05-28 T 14: 13: 49$ \\
14 & Spectrometer Frequency & 400.11 \\
15 & Spectral Width & 6000.6 \\
16 & Lowest Frequency & -992.1 \\
17 & Nucleus & $1 \mathrm{H}$ \\
18 & Acquired Size & 16384 \\
19 & Spectral Size & 65536 \\
\hline
\end{tabular}

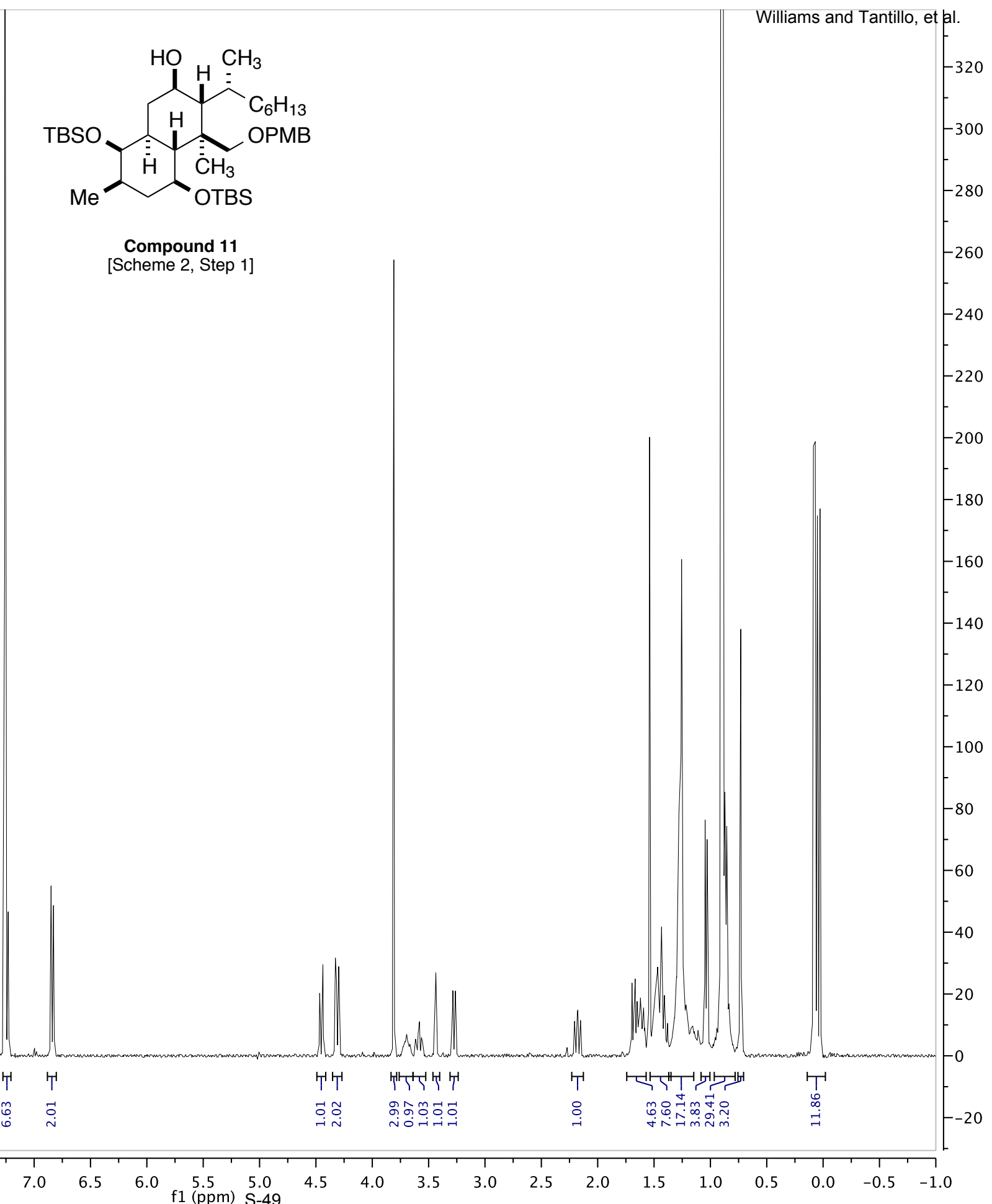

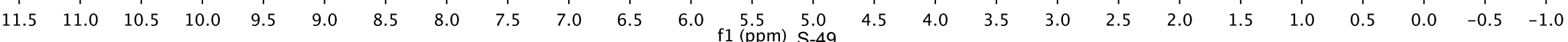




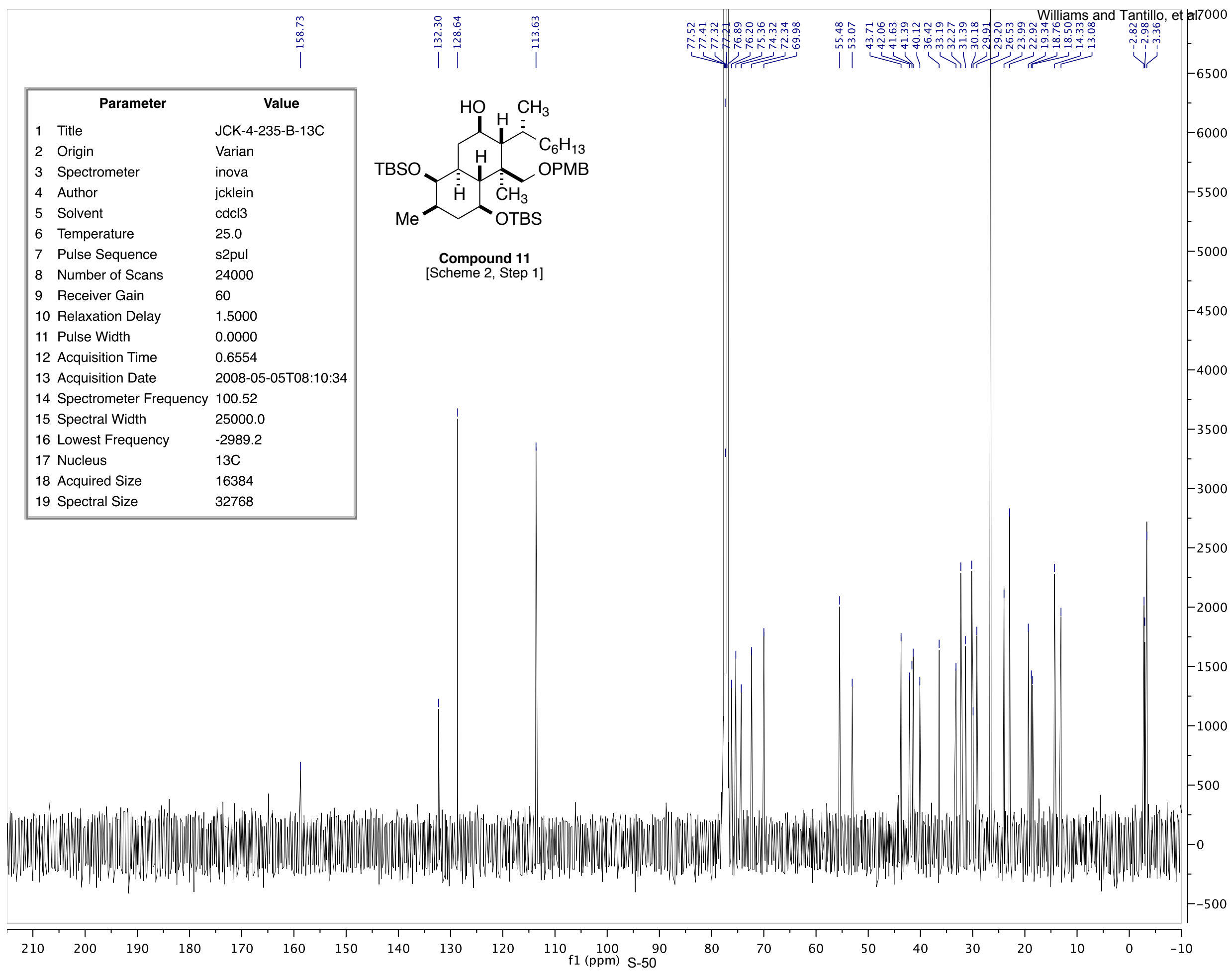




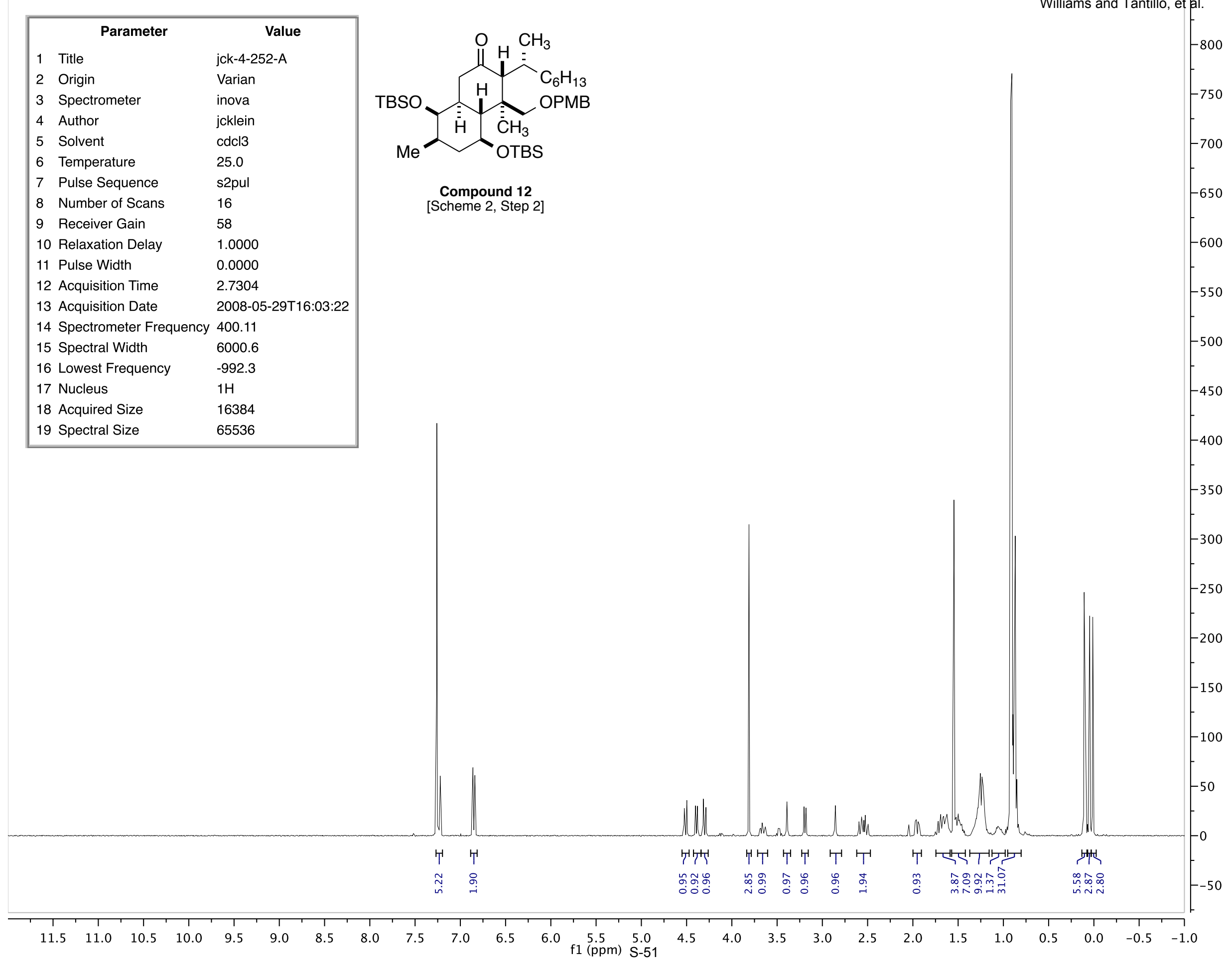




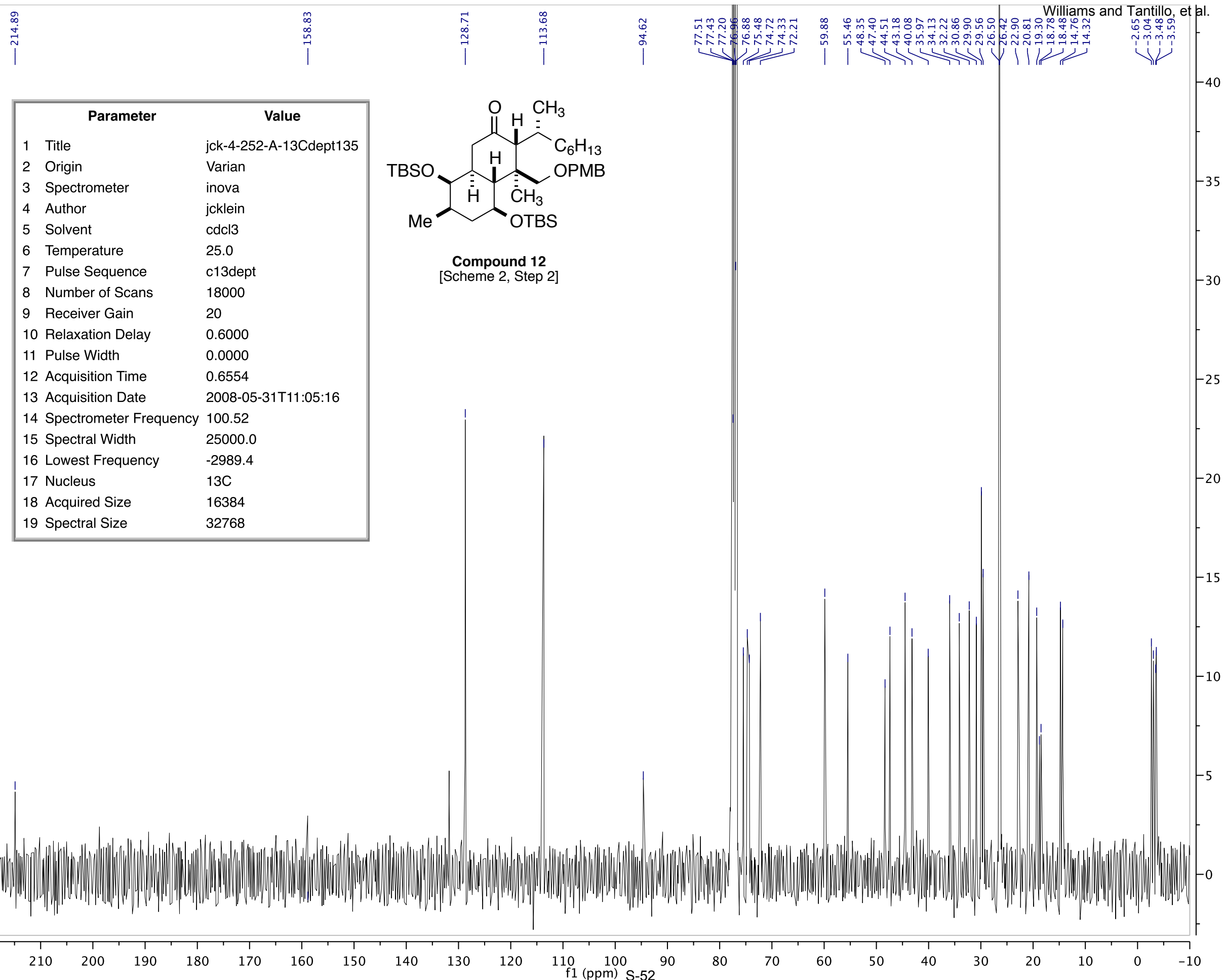




\begin{tabular}{|lll|}
\hline \multicolumn{1}{|c|}{ Parameter } & \multicolumn{1}{c|}{ Value } \\
1 & Title & I-lck-299H_B_f15-21 \\
2 & Origin & Varian \\
3 & Spectrometer & inova \\
4 & Author & jcklein \\
5 & Solvent & cdcl3 \\
6 & Temperature & 25.0 \\
7 & Pulse Sequence & s2pul \\
8 & Number of Scans & 0 \\
9 & Receiver Gain & 36 \\
10 & Relaxation Delay & 1.0000 \\
11 & Pulse Width & 0.0000 \\
12 & Acquisition Time & 2.7304 \\
13 & Acquisition Date & $2009-06-22 T 20: 02: 48$ \\
14 & Spectrometer Frequency & 400.11 \\
15 & Spectral Width & 6000.6 \\
16 & Lowest Frequency & -997.8 \\
17 & Nucleus & $1 \mathrm{H}$ \\
18 & Acquired Size & 16384 \\
19 & Spectral Size & 65536 \\
\hline
\end{tabular}

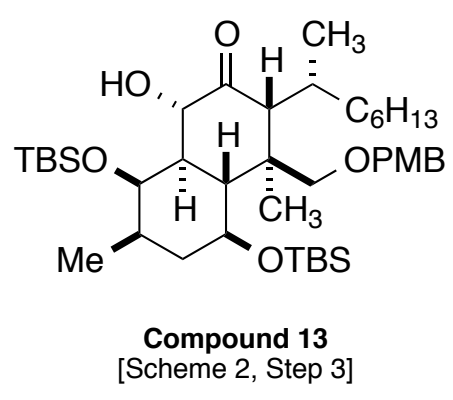

Scheme 2, Step 3

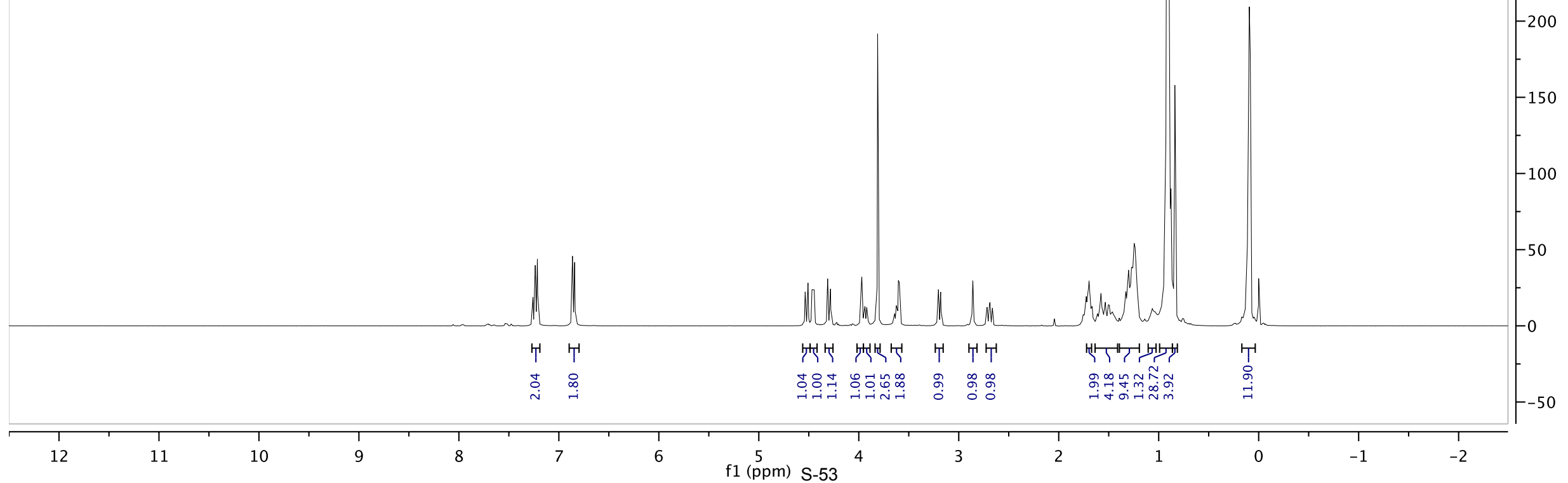




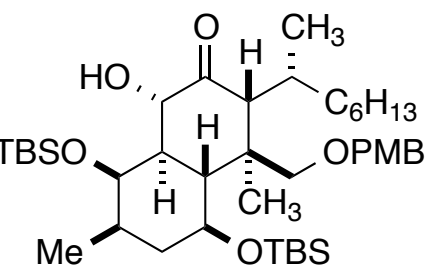

Compound 13

Scheme 2, Step 3]

\begin{tabular}{|lll|}
\hline \multicolumn{1}{|c|}{ Parameter } & \multicolumn{1}{c|}{ Value } \\
1 & Title & I-lck-299H_B_f15-21_gDQCOSY \\
2 & Origin & Varian \\
3 & Spectrometer & inova \\
4 & Author & jcklein \\
5 & Solvent & cdcl3 \\
6 & Temperature & 25.0 \\
7 & Pulse Sequence & gDQCOSY \\
8 & Number of Scans & 0 \\
9 & Receiver Gain & 36 \\
10 & Relaxation Delay & 1.0000 \\
11 & Pulse Width & 0.0000 \\
12 & Acquisition Time & 0.1706 \\
13 & Acquisition Date & $2009-06-22 T 20: 21: 19$ \\
14 & Spectrometer Frequency & $(400.11,400.11)$ \\
15 & Spectral Width & $(6000.6,6000.6)$ \\
16 & Lowest Frequency & $(-997.8,-997.8)$ \\
17 & Nucleus & $(1 \mathrm{H}, 1 \mathrm{H})$ \\
18 & Acquired Size & $(1024,200)$ \\
19 & Spectral Size & $(1024,1024)$ \\
\hline
\end{tabular}

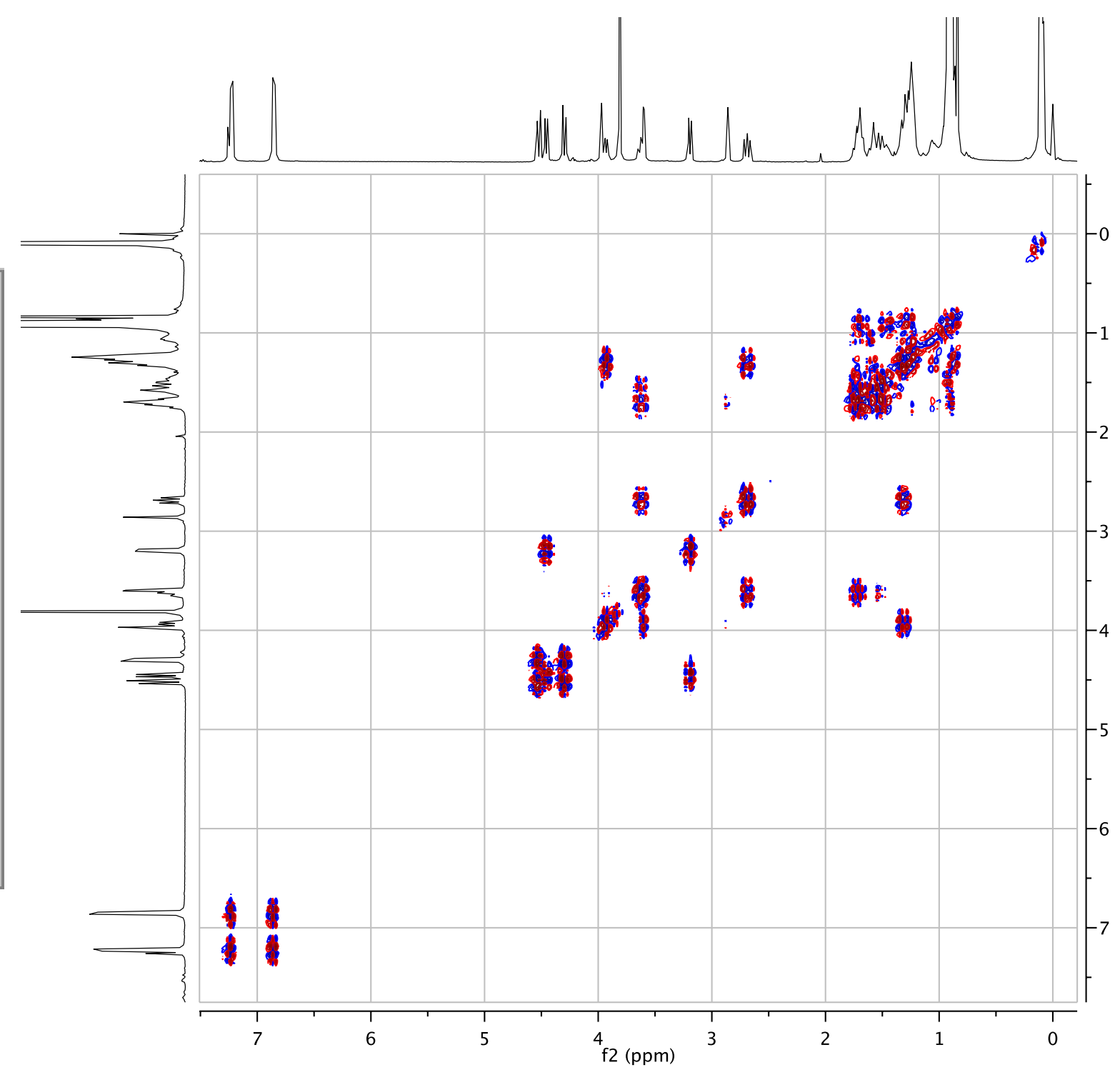




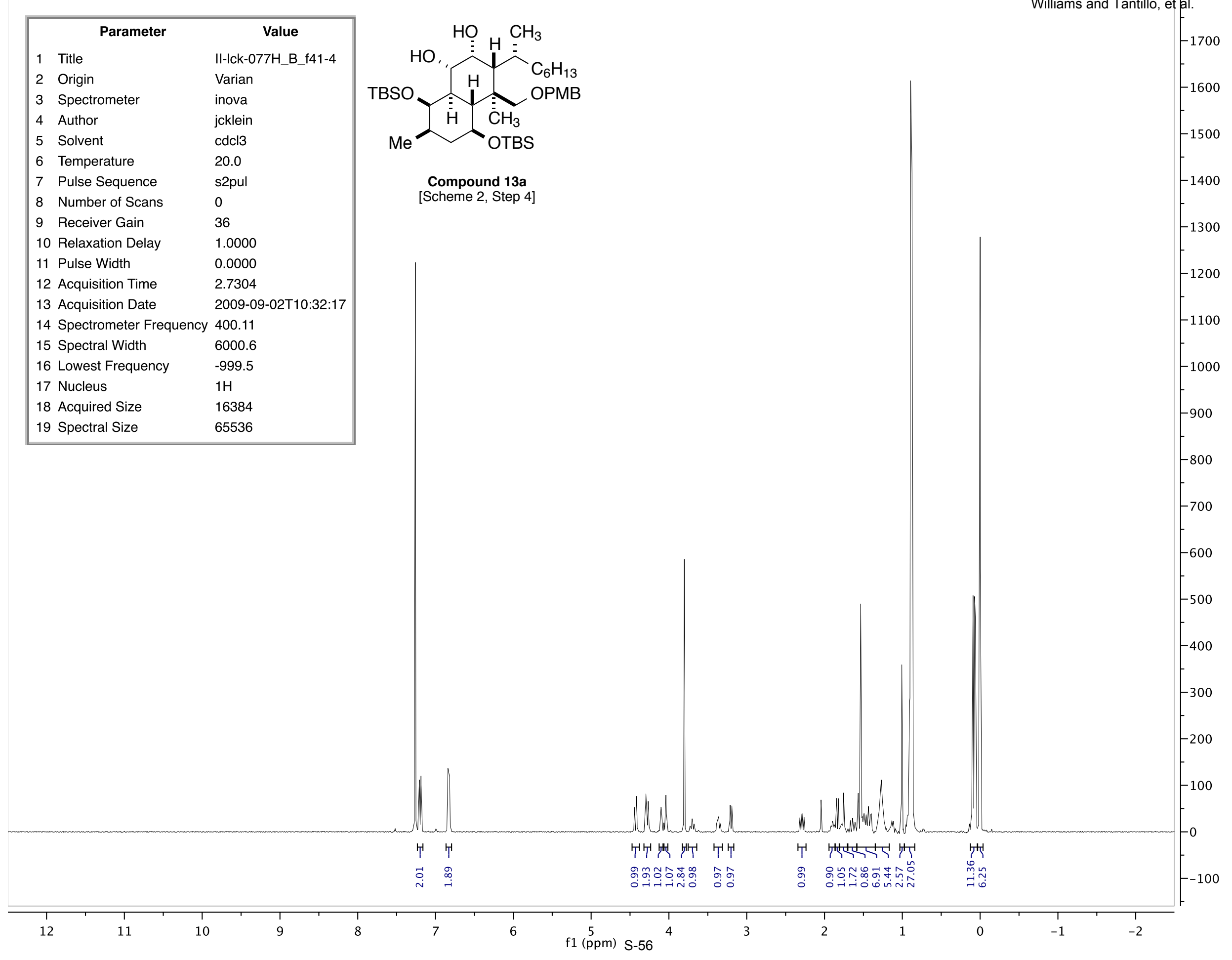




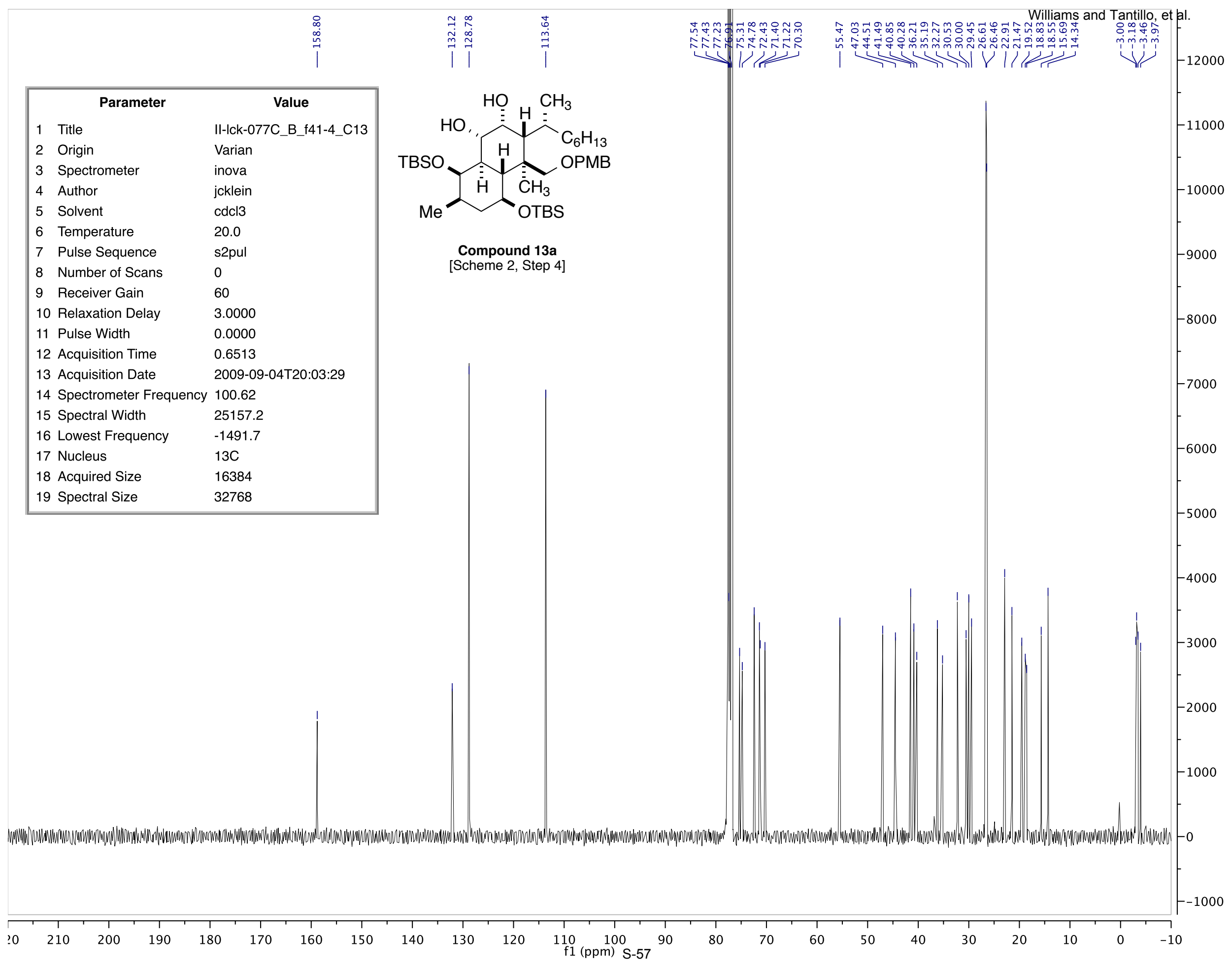




\begin{tabular}{|lll|}
\hline \multicolumn{1}{|c|}{ Parameter } & \multicolumn{1}{c|}{ Value } \\
1 & Title & III-lck-099H_f15-19_col2_forCOSY_vi500 \\
2 & Origin & Varian \\
3 & Spectrometer & inova \\
4 & Author & jcklein \\
5 & Solvent & cdcl3 \\
6 & Temperature & 25.0 \\
7 & Pulse Sequence & s2pul \\
8 & Number of Scans & 24 \\
9 & Receiver Gain & 52 \\
10 & Relaxation Delay & 1.0000 \\
11 & Pulse Width & 0.0000 \\
12 & Acquisition Time & 1.9824 \\
13 & Acquisition Date & $2010-05-29 T 14: 24: 11$ \\
14 & Spectrometer Frequency & 499.81 \\
15 & Spectral Width & 4132.4 \\
16 & Lowest Frequency & -209.9 \\
17 & Nucleus & $1 \mathrm{H}$ \\
18 & Acquired Size & 8192 \\
19 & Spectral Size & 65536 \\
\hline
\end{tabular}

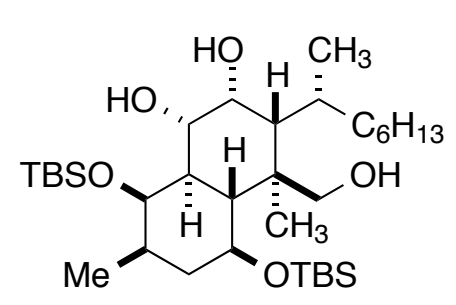

Williams and Tantillo, et al.

Compound 14

[Scheme 2, Step 5]

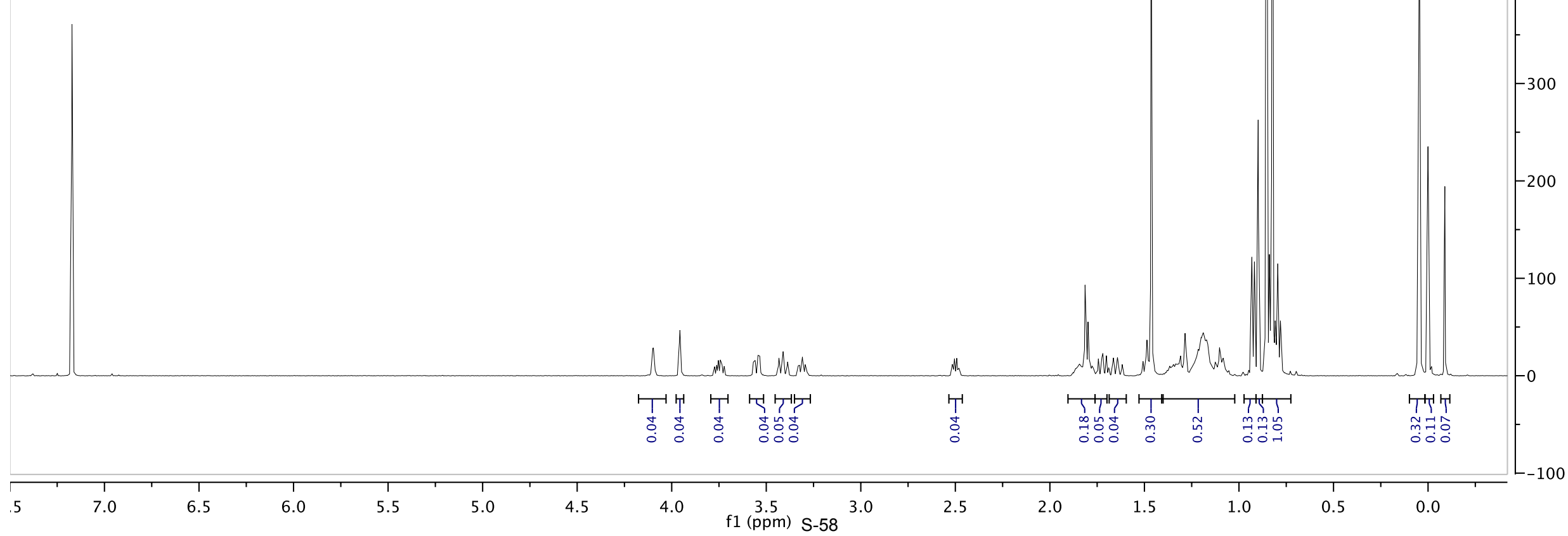




\begin{tabular}{|c|c|c|}
\hline & Parameter & Value \\
\hline 1 & Title & III-Ick-007-013C_prod_C13_vxr \\
\hline 2 & Origin & Varian \\
\hline 3 & Spectrometer & inova \\
\hline 4 & Author & jcklein \\
\hline 5 & Solvent & cdcl3 \\
\hline 6 & Temperature & 25.0 \\
\hline 7 & Pulse Sequence & s2pul \\
\hline 8 & Number of Scans & 1696 \\
\hline 9 & Receiver Gain & 60 \\
\hline 10 & Relaxation Delay & 3.0000 \\
\hline 11 & Pulse Width & 0.0000 \\
\hline 12 & Acquisition Time & 1.3033 \\
\hline 13 & Acquisition Date & 2010-03-27T14:03:19 \\
\hline 14 & Spectrometer Frequency & 100.57 \\
\hline 15 & Spectral Width & 25141.4 \\
\hline 16 & Lowest Frequency & -1508.9 \\
\hline 17 & Nucleus & $13 \mathrm{C}$ \\
\hline 18 & Acquired Size & 32768 \\
\hline 19 & Spectral Size & 65536 \\
\hline
\end{tabular}

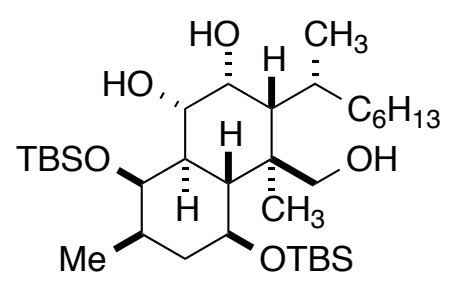

Compound 14

[Scheme 2, Step 5]

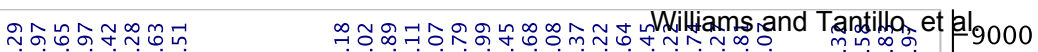

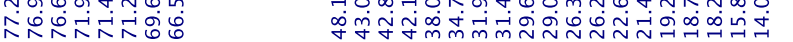

$\sqrt[1]{+}$
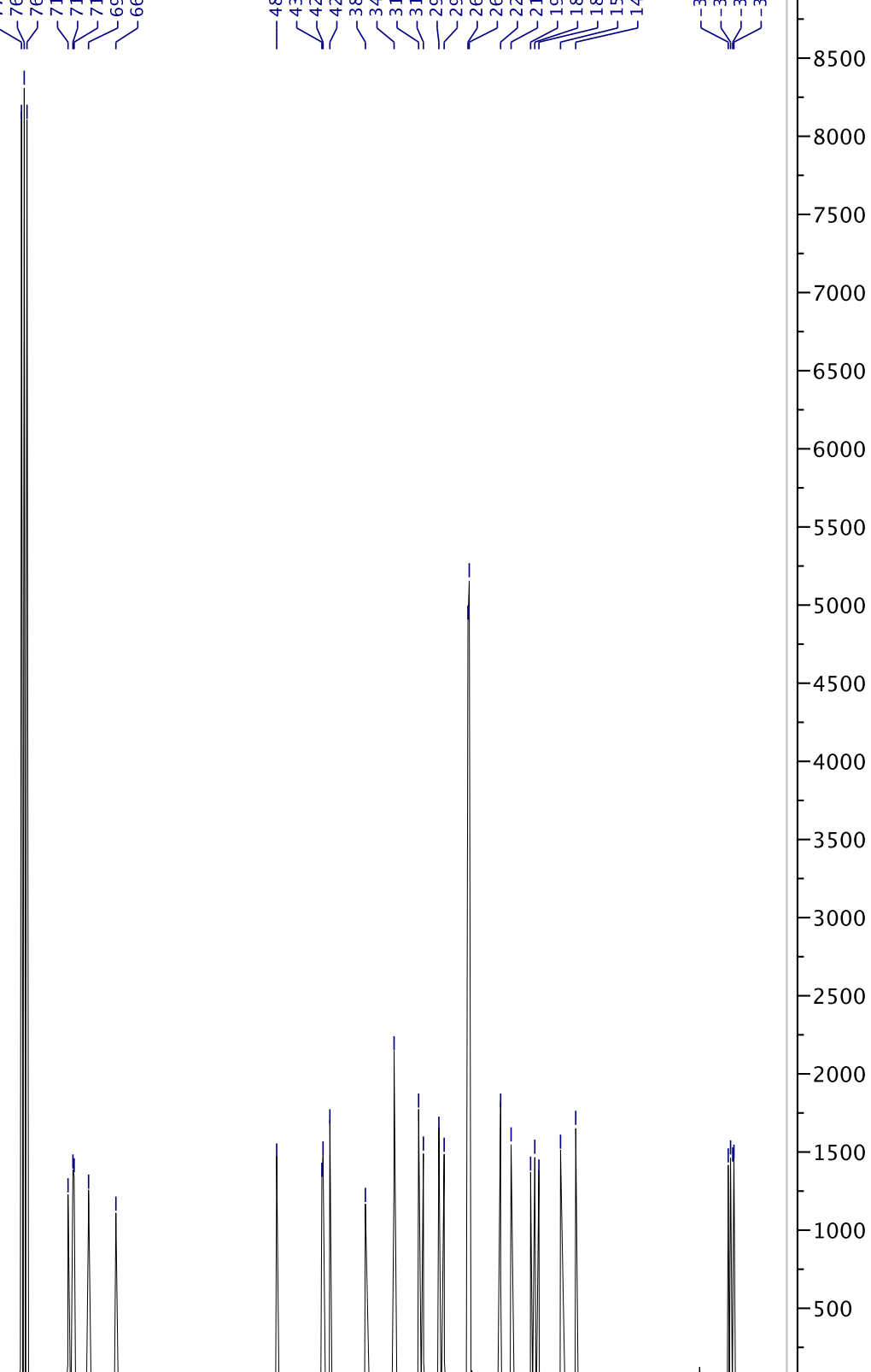

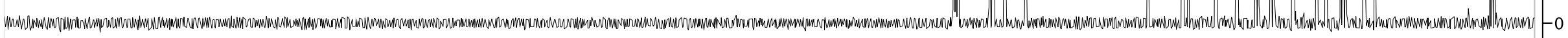

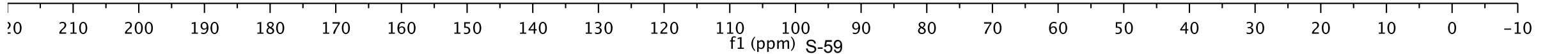




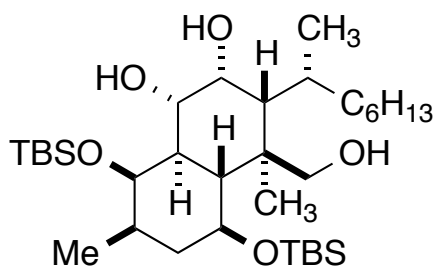

Compound 14

[Scheme 2, Step 5]

\begin{tabular}{|lll|}
\hline \multicolumn{1}{|c|}{ Parameter } & \multicolumn{1}{c|}{ Value } \\
1 & Title & IV-lck-149H_f14-20_wD2O_COSY \\
2 & Origin & Varian \\
3 & Spectrometer & inova \\
4 & Author & jcklein \\
5 & Solvent & cdcl3 \\
6 & Temperature & 25.0 \\
7 & Pulse Sequence & gCOSY \\
8 & Number of Scans & 4 \\
9 & Receiver Gain & 58 \\
10 & Relaxation Delay & 1.0000 \\
11 & Pulse Width & 0.0000 \\
12 & Acquisition Time & 0.1500 \\
13 & Acquisition Date & $2011-05-21 T 19: 23: 52$ \\
14 & Spectrometer Frequency & $(400.11,400.11)$ \\
15 & Spectral Width & $(3472.4,3472.4)$ \\
16 & Lowest Frequency & $(-240.4,-240.4)$ \\
17 & Nucleus & $(1 \mathrm{H}, 1 \mathrm{H})$ \\
18 & Acquired Size & $(521,128)$ \\
19 & Spectral Size & $(1024,1024)$ \\
\hline
\end{tabular}

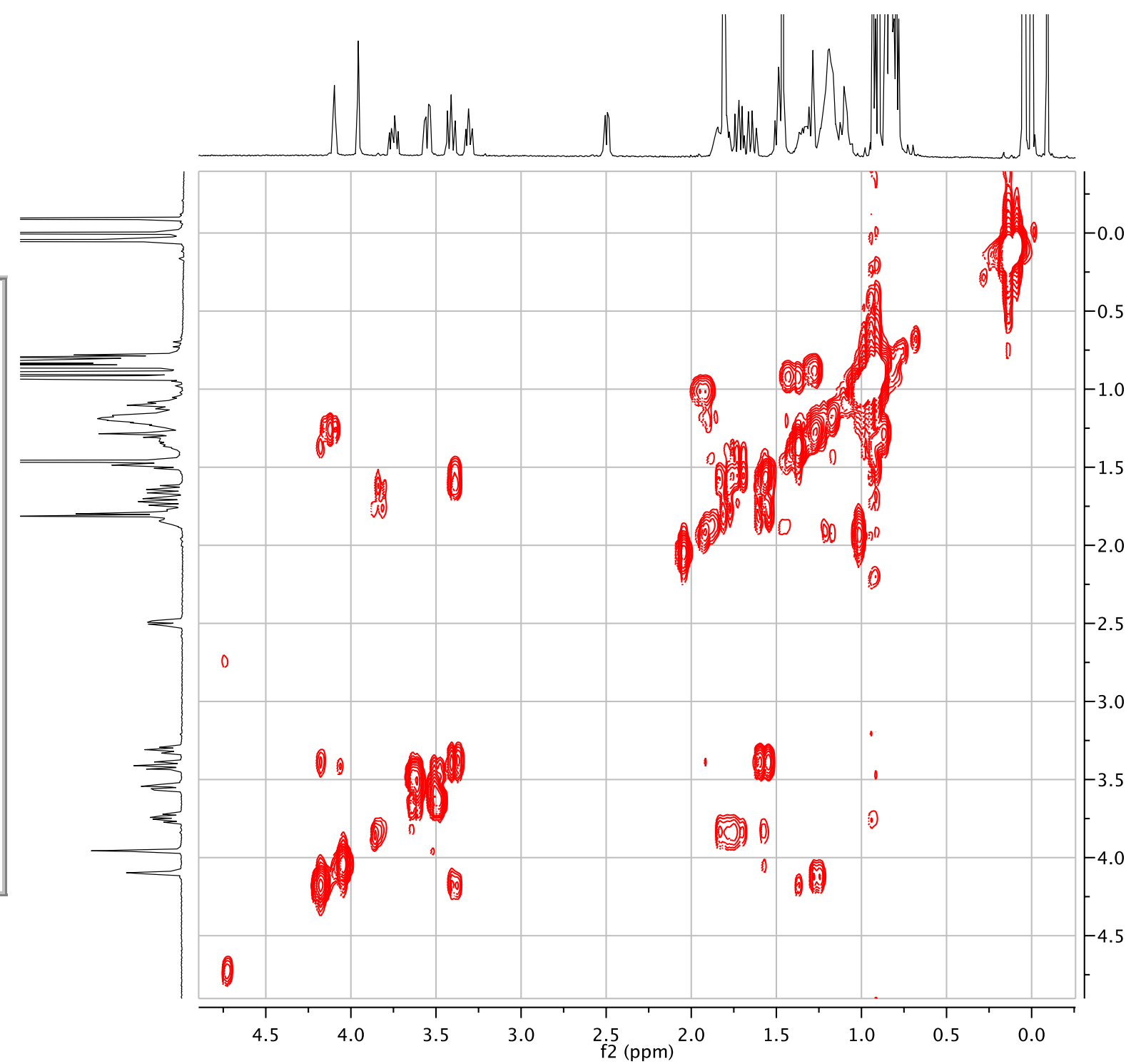




\begin{tabular}{|lll|}
\hline \multicolumn{1}{|c|}{ Parameter } & \multicolumn{1}{c|}{ Value } \\
1 & Title & III-lck-115H_f14-17_vi500 \\
2 & Origin & Varian \\
3 & Spectrometer & inova \\
4 & Author & jcklein \\
5 & Solvent & cdcl3 \\
6 & Temperature & 25.0 \\
7 & Pulse Sequence & s2pul \\
8 & Number of Scans & 48 \\
9 & Receiver Gain & 46 \\
10 & Relaxation Delay & 1.0000 \\
11 & Pulse Width & 0.0000 \\
12 & Acquisition Time & 2.0488 \\
13 & Acquisition Date & $2010-06-16 T 10: 59: 21$ \\
14 & Spectrometer Frequency & 499.81 \\
15 & Spectral Width & 7996.8 \\
16 & Lowest Frequency & -999.6 \\
17 & Nucleus & $1 \mathrm{H}$ \\
18 & Acquired Size & 16384 \\
19 & Spectral Size & 65536 \\
\hline
\end{tabular}

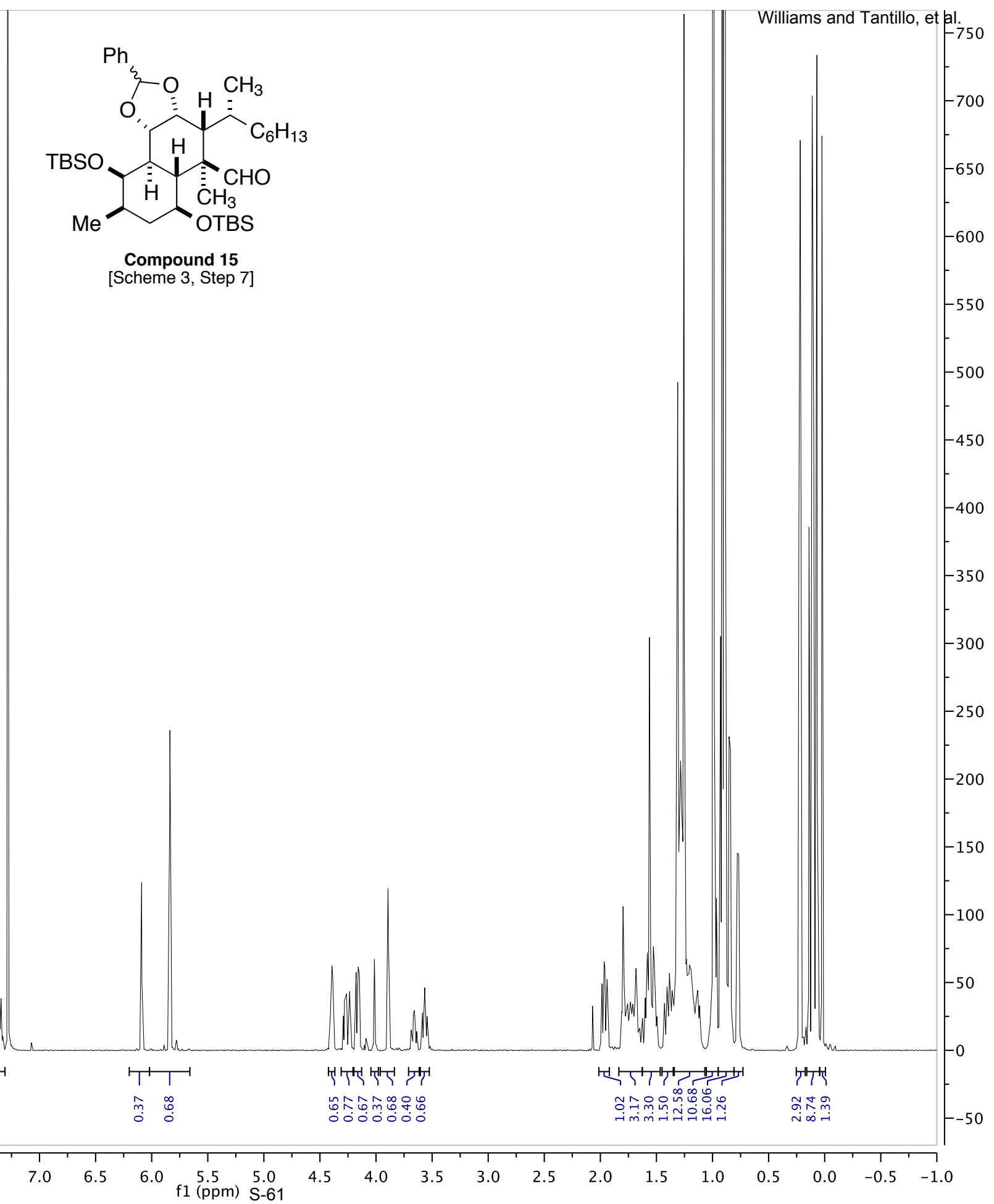




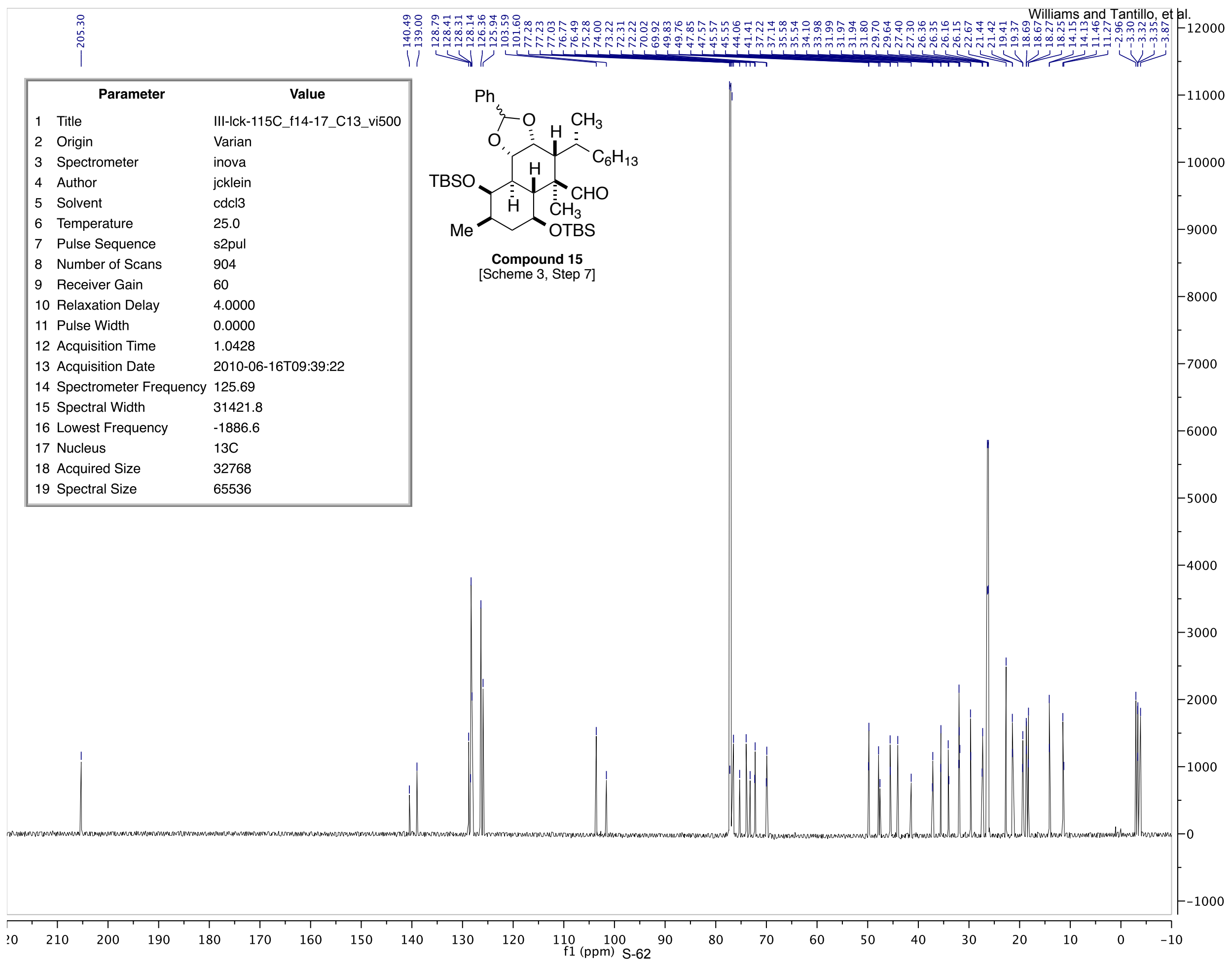




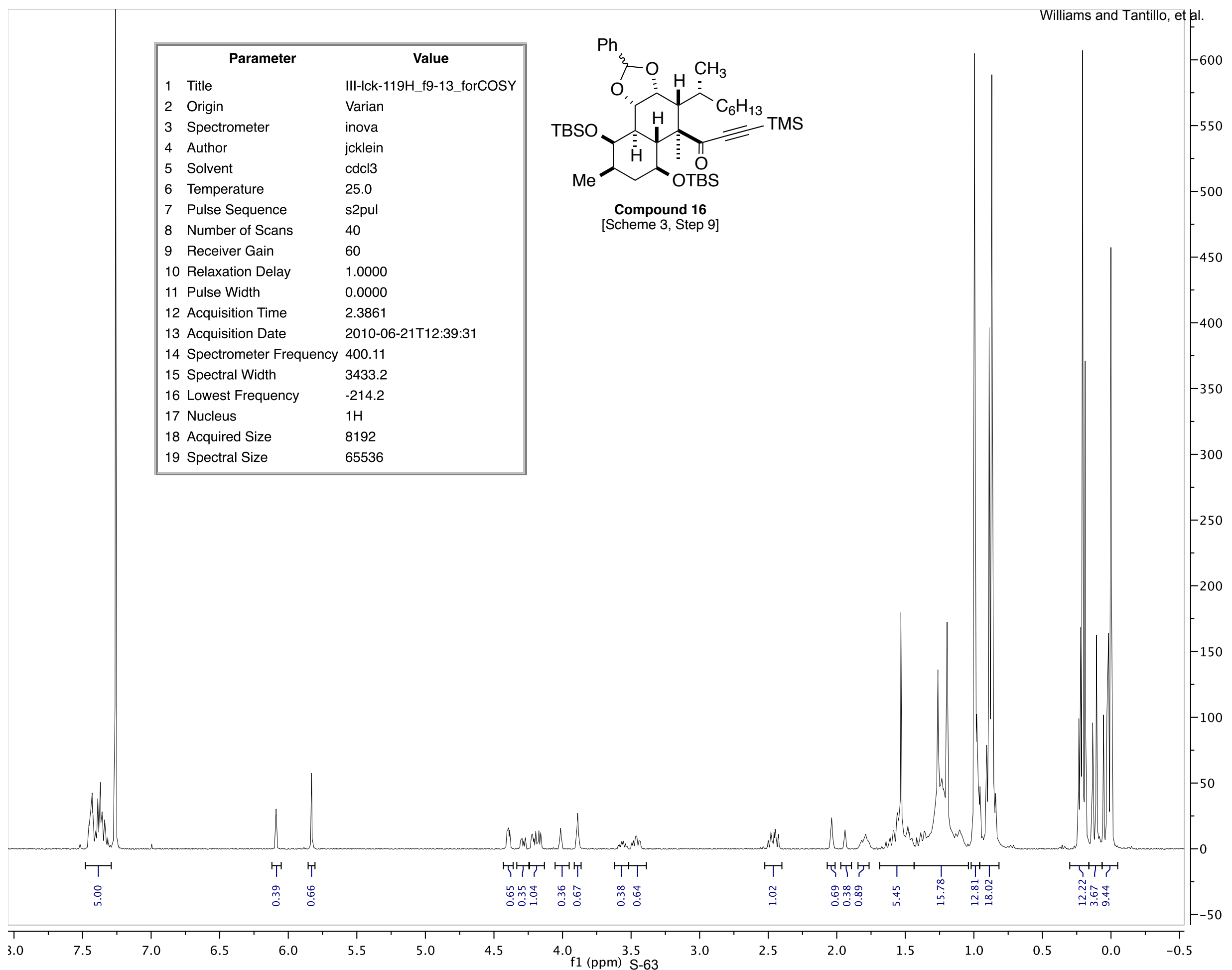




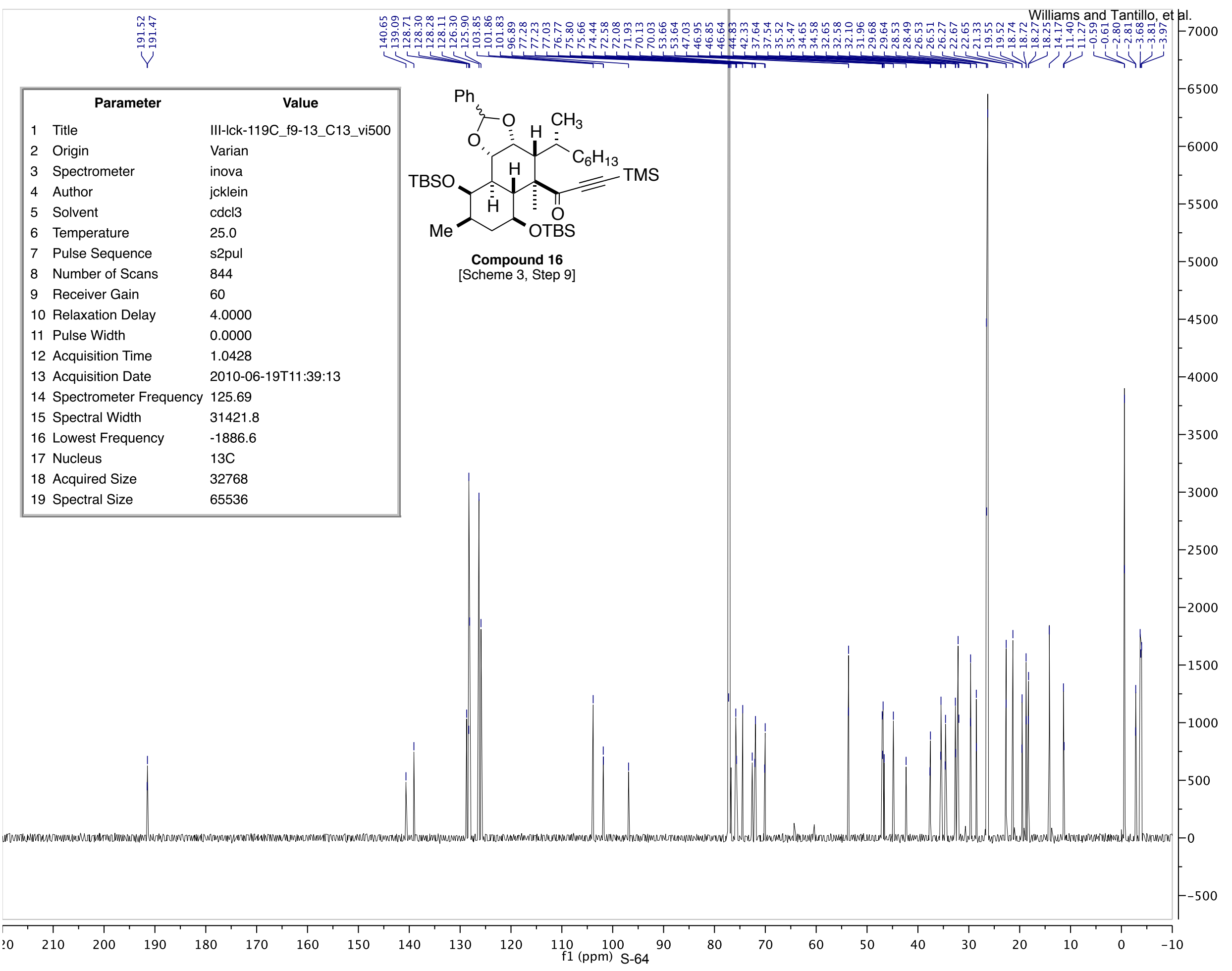




\begin{tabular}{|lll|}
\hline \multicolumn{1}{|c|}{ Parameter } & \multicolumn{1}{c|}{ Value } \\
1 & Title & IV-lck-159H_crude_i500 \\
2 & Origin & Varian \\
3 & Spectrometer & inova \\
4 & Author & jcklein \\
5 & Solvent & cdcl3 \\
6 & Temperature & 26.0 \\
7 & Pulse Sequence & s2pul \\
8 & Number of Scans & 24 \\
9 & Receiver Gain & 58 \\
10 & Relaxation Delay & 1.0000 \\
11 & Pulse Width & 0.0000 \\
12 & Acquisition Time & 2.0488 \\
13 & Acquisition Date & $2011-05-29 T 16: 26: 48$ \\
14 & Spectrometer Frequency & 499.81 \\
15 & Spectral Width & 7996.8 \\
16 & Lowest Frequency & -999.6 \\
17 & Nucleus & $1 \mathrm{H}$ \\
18 & Acquired Size & 16384 \\
19 & Spectral Size & 65536 \\
\hline
\end{tabular}

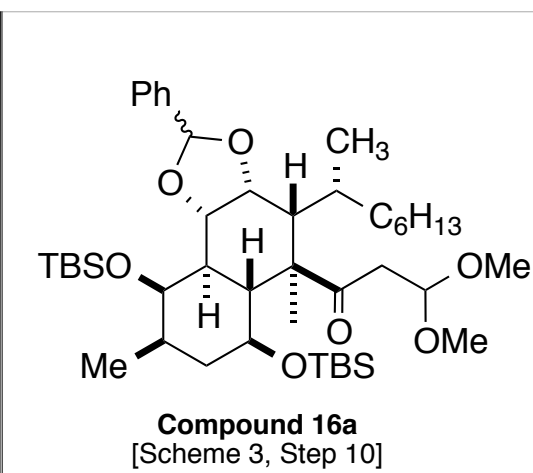

Williams and Tantillo, et al.

Scheme 3, Step 10

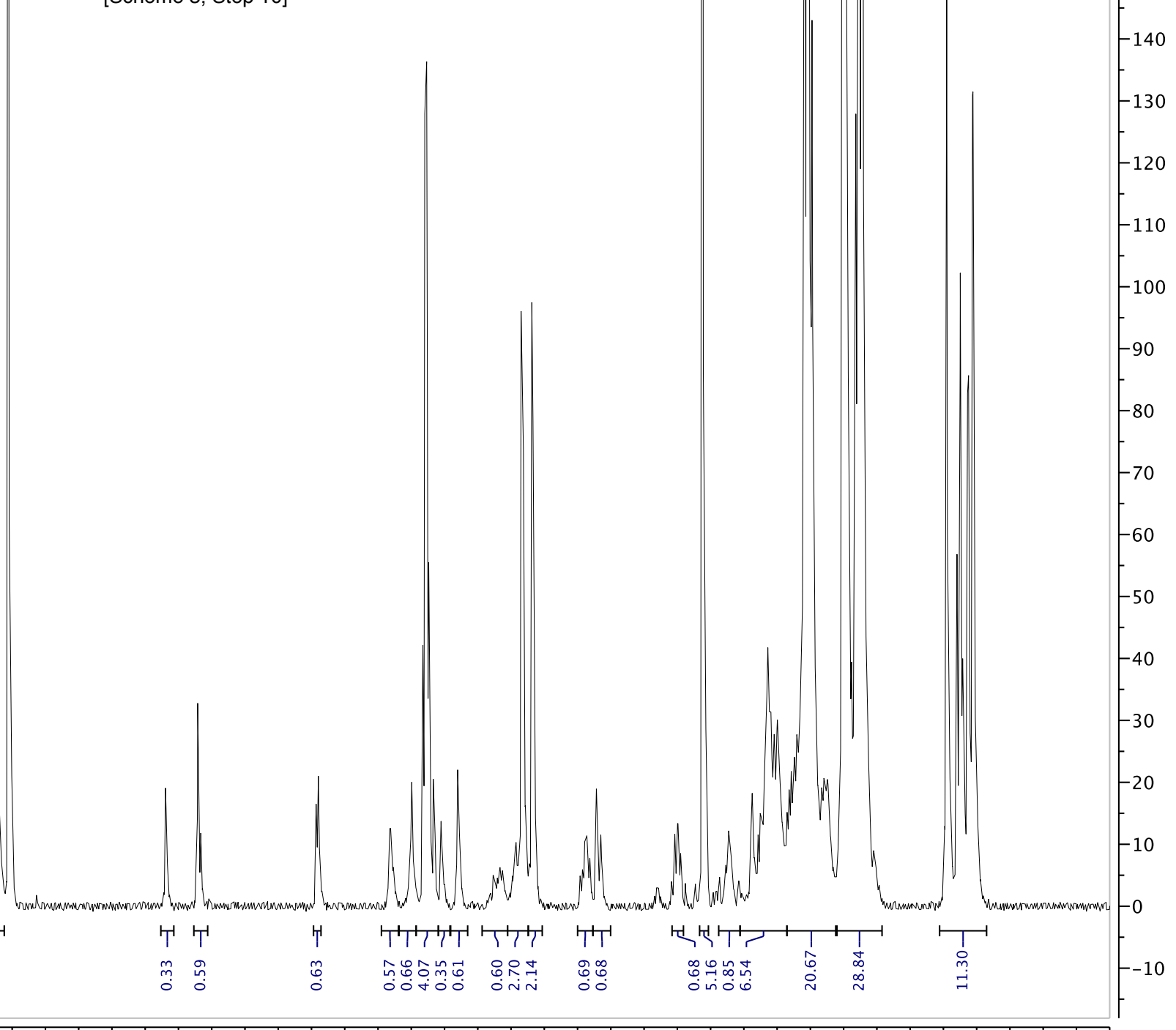

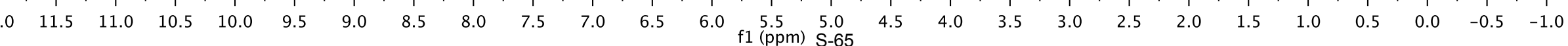




\begin{tabular}{|lll|}
\hline \multicolumn{1}{|c|}{ Parameter } & \multicolumn{1}{c|}{ Value } \\
1 & Title & III-lck-123H_B_f30-43_vi500 \\
2 & Origin & Varian \\
3 & Spectrometer & inova \\
4 & Author & jcklein \\
5 & Solvent & cdcl3 \\
6 & Temperature & 25.0 \\
7 & Pulse Sequence & s2pul \\
8 & Number of Scans & 36 \\
9 & Receiver Gain & 26 \\
10 & Relaxation Delay & 1.0000 \\
11 & Pulse Width & 0.0000 \\
12 & Acquisition Time & 2.0488 \\
13 & Acquisition Date & $2010-06-29 T 09: 31: 31$ \\
14 & Spectrometer Frequency & 499.81 \\
15 & Spectral Width & 7996.8 \\
16 & Lowest Frequency & -999.6 \\
17 & Nucleus & $1 \mathrm{H}$ \\
18 Acquired Size & 16384 \\
19 & Spectral Size & 65536 \\
\hline
\end{tabular}

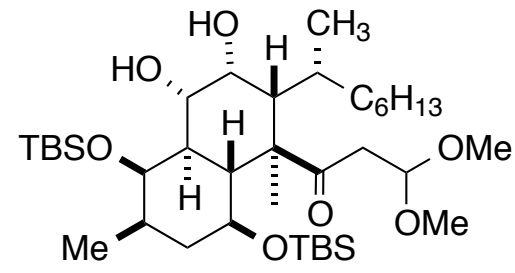

Williams and Tantillo, et al.

Compound 17

[Scheme 3, Step 11]

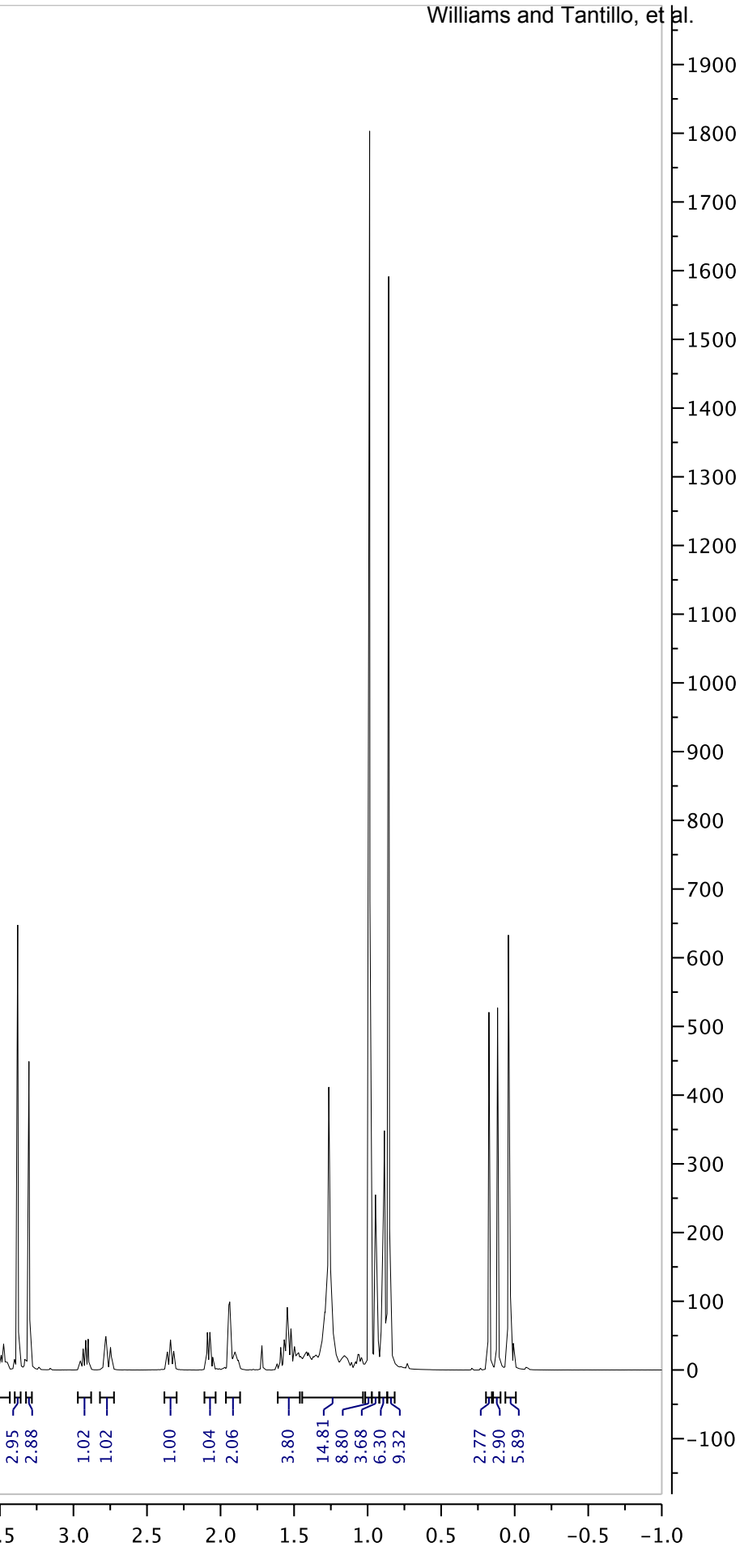




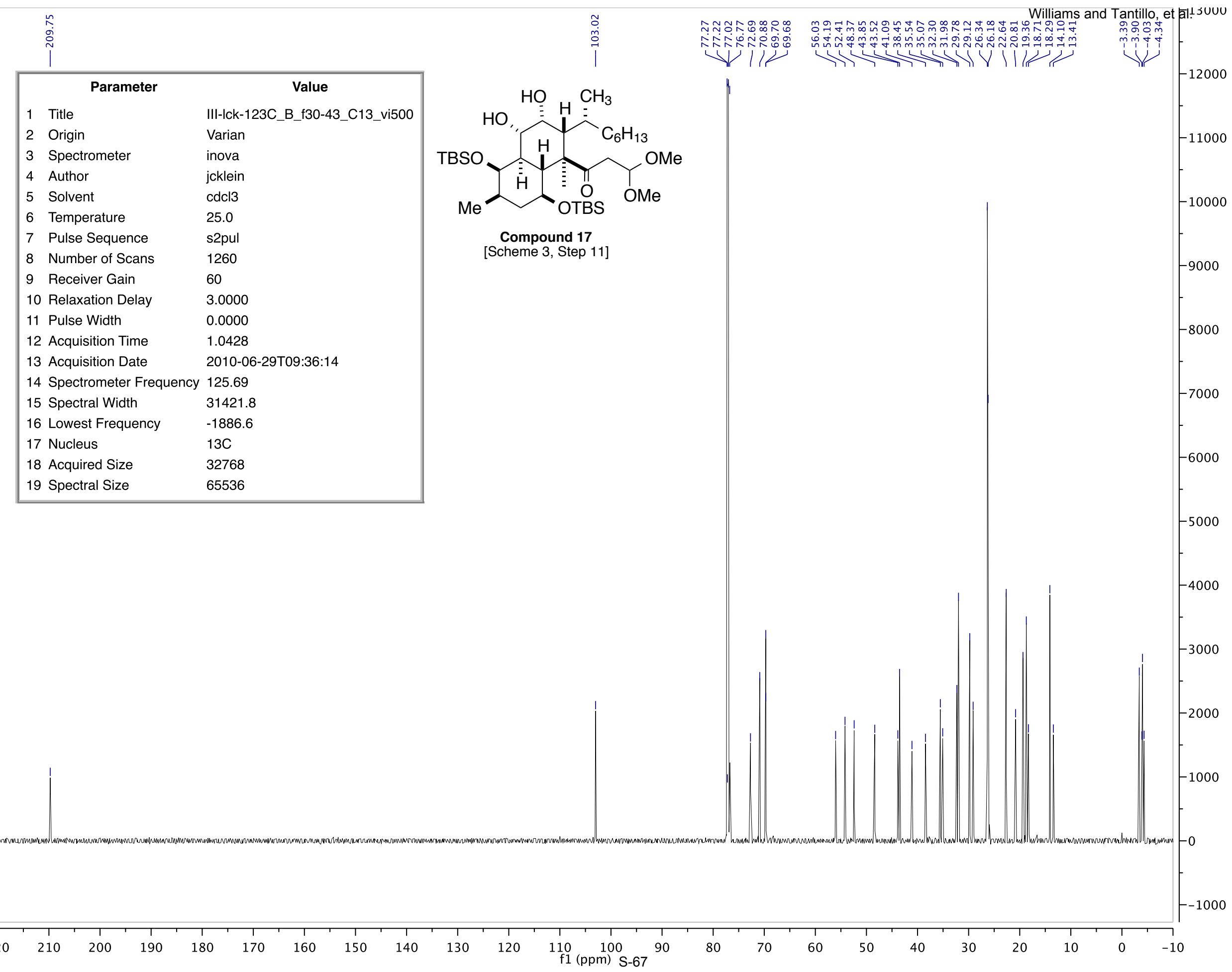




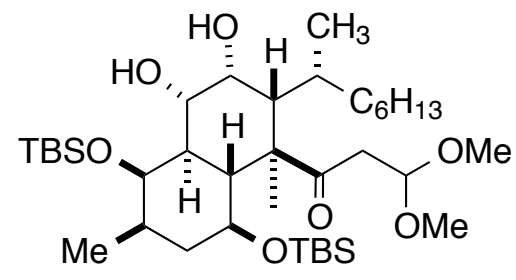

Compound 17

[Scheme 3, Step 11]

\begin{tabular}{|lll|}
\hline \multicolumn{2}{|c|}{ Parameter } & \multicolumn{1}{c|}{ Value } \\
1 & Title & III-Ick-123H_B_f30-43_COSY_vi500 \\
2 & Origin & Varian \\
3 & Spectrometer & inova \\
4 & Author & jcklein \\
5 & Solvent & cdcl3 \\
6 & Temperature & 25.0 \\
7 & Pulse Sequence & gCOSY \\
8 & Number of Scans & 4 \\
9 & Receiver Gain & 26 \\
10 & Relaxation Delay & 1.0000 \\
11 & Pulse Width & 0.0000 \\
12 & Acquisition Time & 0.1499 \\
13 & Acquisition Date & $2010-06-29 \mathrm{~T} 11: 17: 39$ \\
14 & Spectrometer Frequency & $(499.81,499.81)$ \\
15 & Spectral Width & $(4088.5,4088.5)$ \\
16 & Lowest Frequency & $(-219.9,-219.9)$ \\
17 & Nucleus & $(1 \mathrm{H}, 1 \mathrm{H})$ \\
18 & Acquired Size & $(613,128)$ \\
19 & Spectral Size & $(1024,1024)$ \\
\hline
\end{tabular}

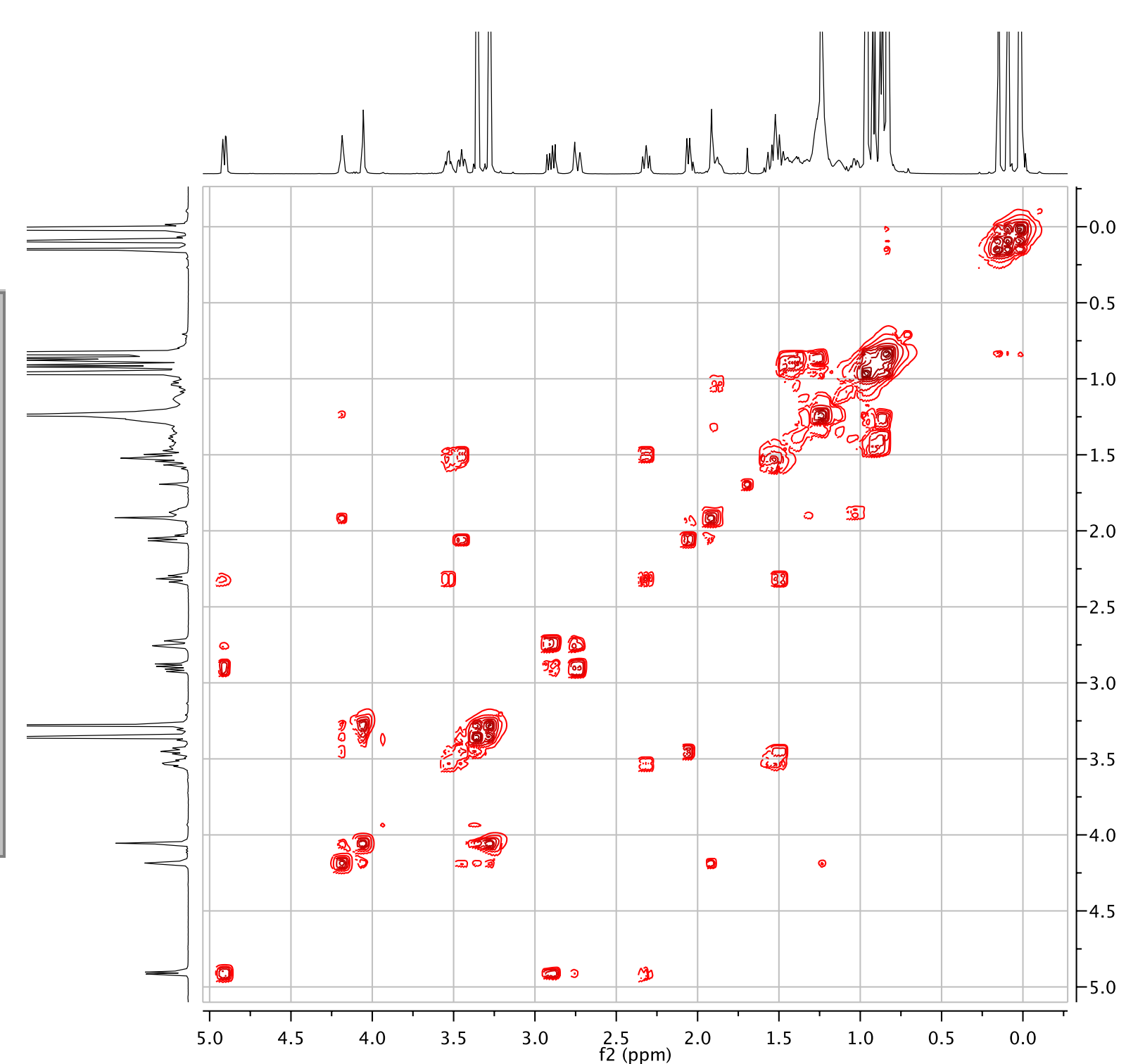




\begin{tabular}{|lll|}
\hline \multicolumn{1}{|c|}{ Parameter } & \multicolumn{1}{c|}{ Value } \\
1 & Title & III-lck-127H_f19-27_forC13_vi500 \\
2 & Origin & Varian \\
3 & Spectrometer & inova \\
4 & Author & jcklein \\
5 & Solvent & cdcl3 \\
6 & Temperature & 25.0 \\
7 & Pulse Sequence & s2pul \\
8 & Number of Scans & 32 \\
9 & Receiver Gain & 36 \\
10 & Relaxation Delay & 1.0000 \\
11 & Pulse Width & 0.0000 \\
12 & Acquisition Time & 2.0488 \\
13 & Acquisition Date & $2010-07-04 T 09: 44: 15$ \\
14 & Spectrometer Frequency & 499.81 \\
15 & Spectral Width & 7996.8 \\
16 & Lowest Frequency & -999.6 \\
17 & Nucleus & $1 \mathrm{H}$ \\
18 Acquired Size & 16384 \\
19 & Spectral Size & 65536 \\
\hline
\end{tabular}

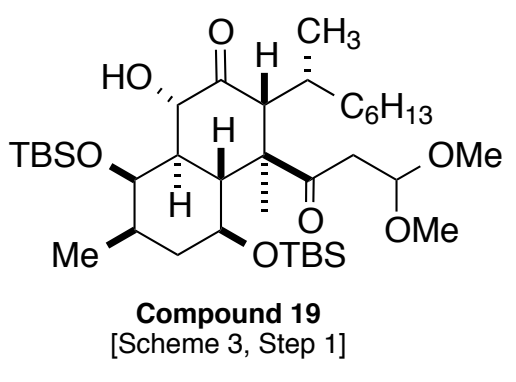

Williams and Tantillo, et al.
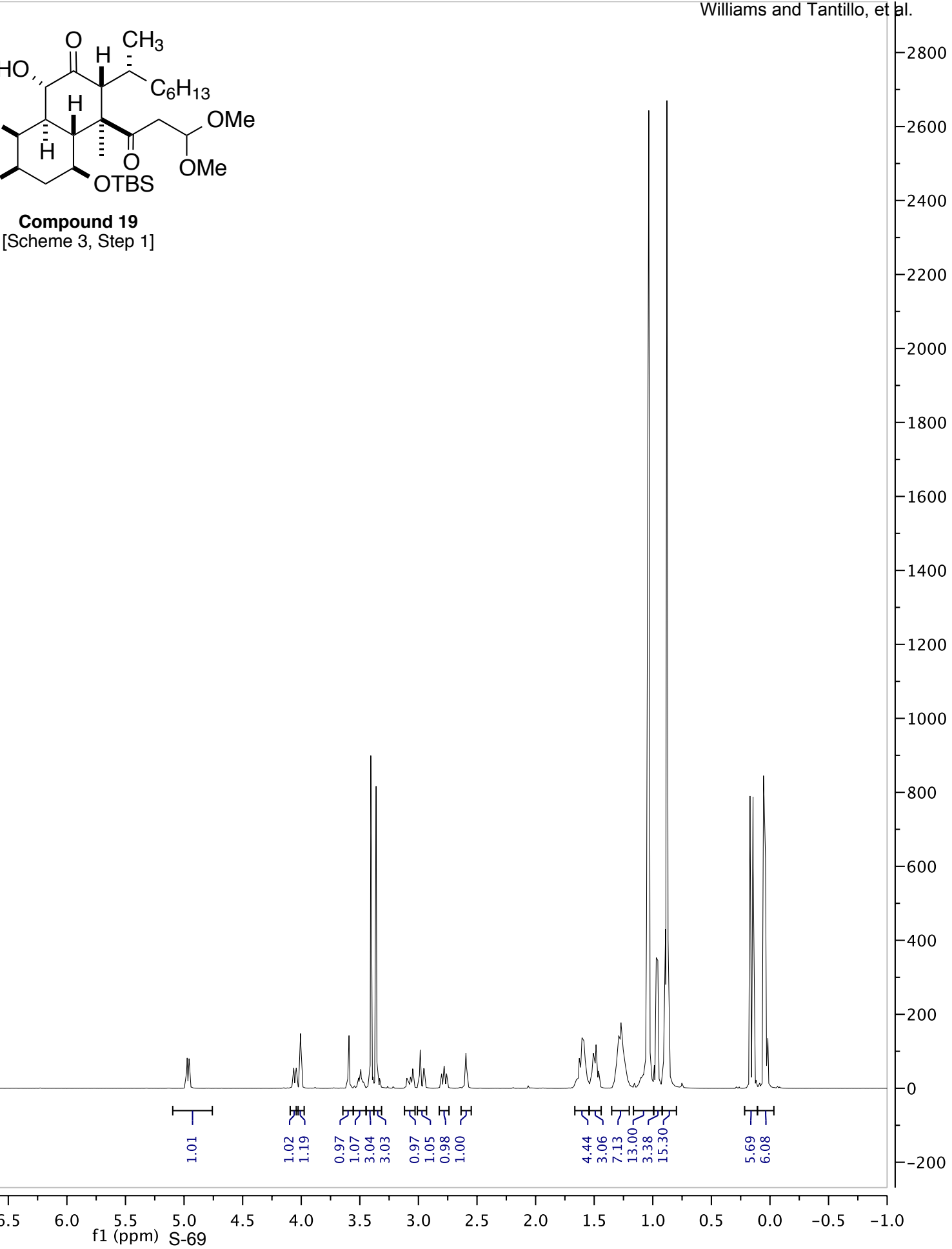


\begin{tabular}{|lll|}
\hline \multicolumn{1}{|c|}{ Parameter } & \multicolumn{1}{c|}{ Value } \\
1 & Title & III-lck-127C_f19-27_C13_vi500 \\
2 & Origin & Varian \\
3 & Spectrometer & inova \\
4 & Author & jcklein \\
5 & Solvent & cdcl3 \\
6 & Temperature & 25.0 \\
7 & Pulse Sequence & s2pul \\
8 & Number of Scans & 4544 \\
9 & Receiver Gain & 60 \\
10 & Relaxation Delay & 3.0000 \\
11 & Pulse Width & 0.0000 \\
12 & Acquisition Time & 1.0428 \\
13 & Acquisition Date & $2010-07-04 T 09: 57: 02$ \\
14 & Spectrometer Frequency & 125.69 \\
15 & Spectral Width & 31421.8 \\
16 & Lowest Frequency & -1886.6 \\
17 & Nucleus & $13 \mathrm{C}$ \\
18 & Acquired Size & 32768 \\
19 & Spectral Size & 65536 \\
\hline
\end{tabular}

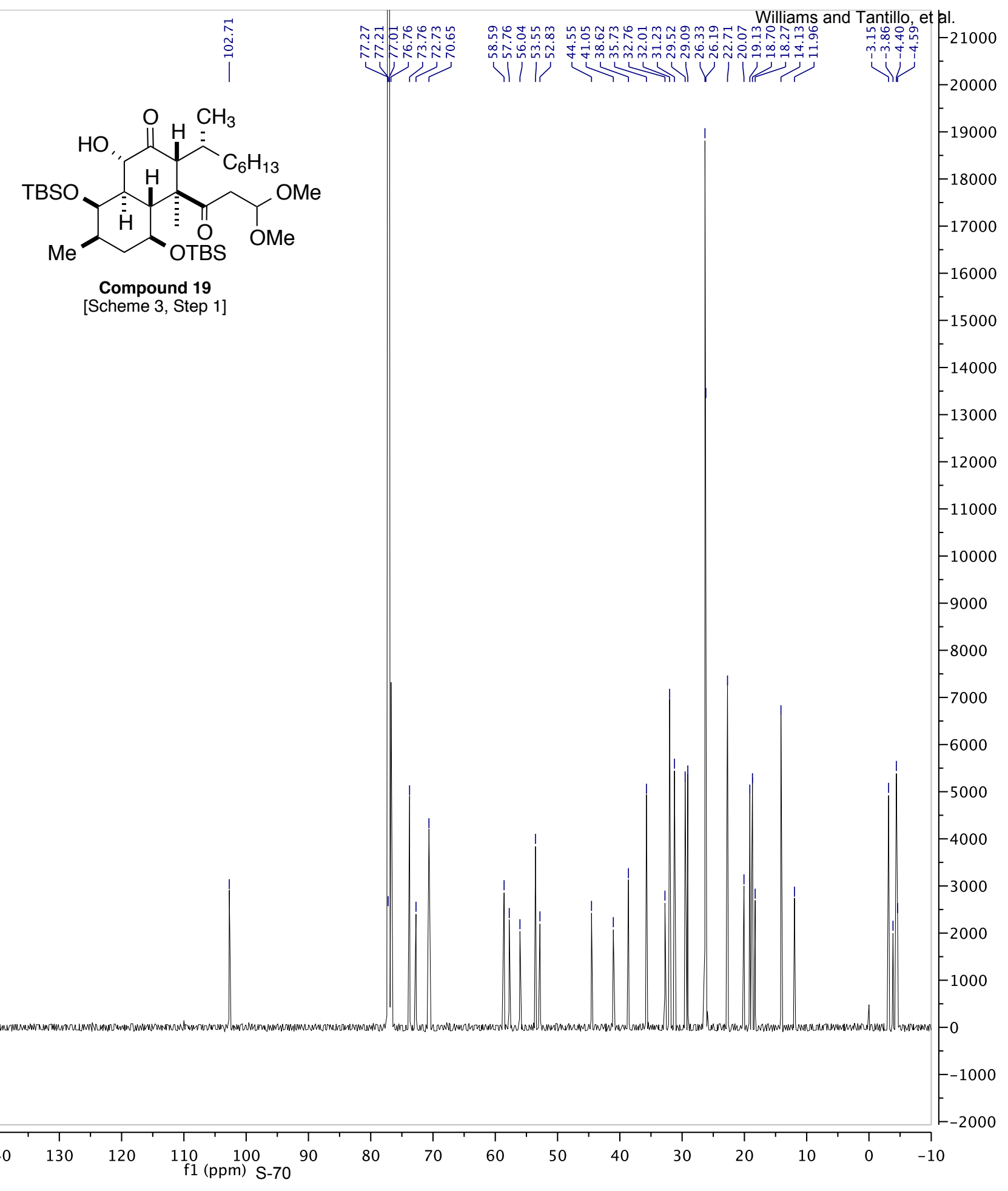

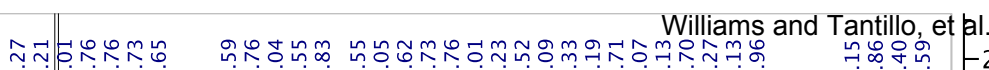

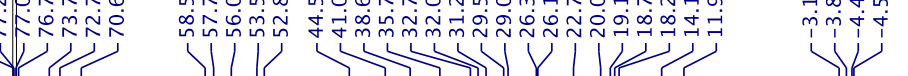

1 Title

Spectromete

3.0000

12 Acquisition Time

13 Acquisition Date

5 Spectral Width

Lowest Frequency

8 Acquired Siz 


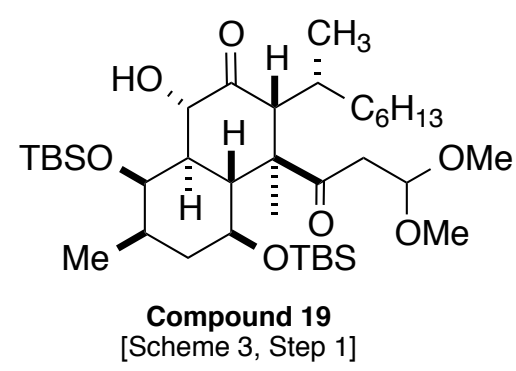

\begin{tabular}{|lll|}
\hline \multicolumn{1}{|c|}{ Parameter } & \multicolumn{1}{c|}{ Value } \\
1 & Title & III-lck-127H_19-27_COSY_vi500 \\
2 & Origin & Varian \\
3 & Spectrometer & inova \\
4 & Author & jcklein \\
5 & Solvent & cdcl3 \\
6 & Temperature & 25.0 \\
7 & Pulse Sequence & gCOSY \\
8 & Number of Scans & 4 \\
9 & Receiver Gain & 32 \\
10 & Relaxation Delay & 1.0000 \\
11 & Pulse Width & 0.0000 \\
12 & Acquisition Time & 0.1500 \\
13 & Acquisition Date & $2010-07-04 T 15: 21: 01$ \\
14 & Spectrometer Frequency & $(499.81,499.81)$ \\
15 & Spectral Width & $(4152.0,4152.0)$ \\
16 & Lowest Frequency & $(-229.4,-229.4)$ \\
17 & Nucleus & $(1 \mathrm{H}, 1 \mathrm{H})$ \\
18 & Acquired Size & $(623,128)$ \\
19 & Spectral Size & $(1024,1024)$ \\
\hline
\end{tabular}

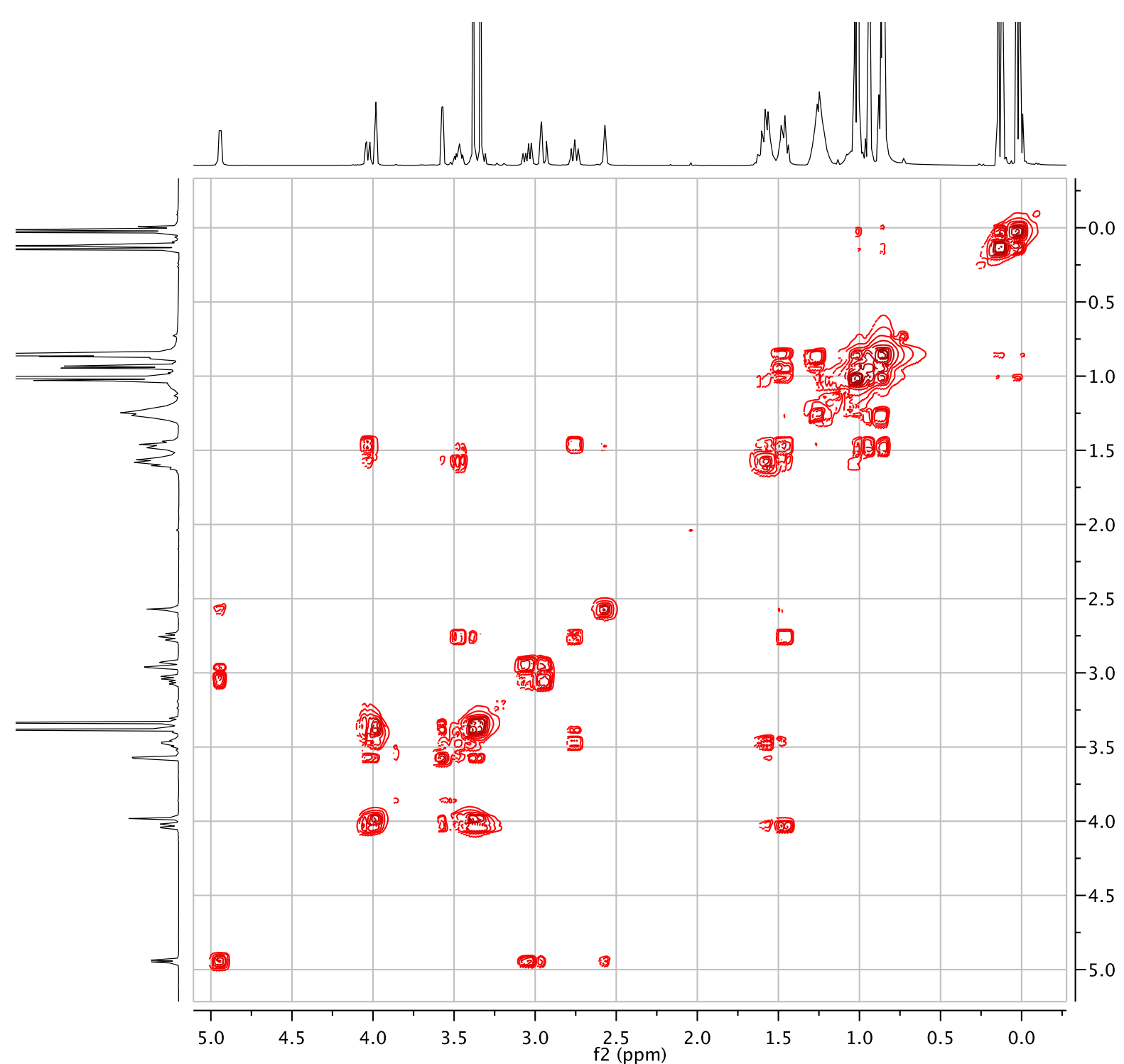




\begin{tabular}{|lll|}
\hline \multicolumn{2}{|c|}{ Parameter } & \multicolumn{1}{c|}{ Value } \\
1 & Title & SAB-4-292 \\
2 & Origin & Varian \\
3 & Spectrometer & inova \\
4 & Solvent & cdcl3 \\
5 & Temperature & 25.0 \\
6 & Pulse Sequence & s2pul \\
7 & Number of Scans & 64 \\
8 & Receiver Gain & 30 \\
9 & Relaxation Delay & 1.0000 \\
10 & Pulse Width & 0.0000 \\
11 & Acquisition Time & 1.7046 \\
12 & Acquisition Date & $2015-10-30 T 19: 27: 06$ \\
13 & Spectrometer Frequency & 599.80 \\
14 & Spectral Width & 9611.9 \\
15 & Lowest Frequency & -1192.2 \\
16 & Nucleus & $1 \mathrm{H}$ \\
17 & Acquired Size & 16384 \\
18 & Spectral Size & 65536 \\
\hline
\end{tabular}

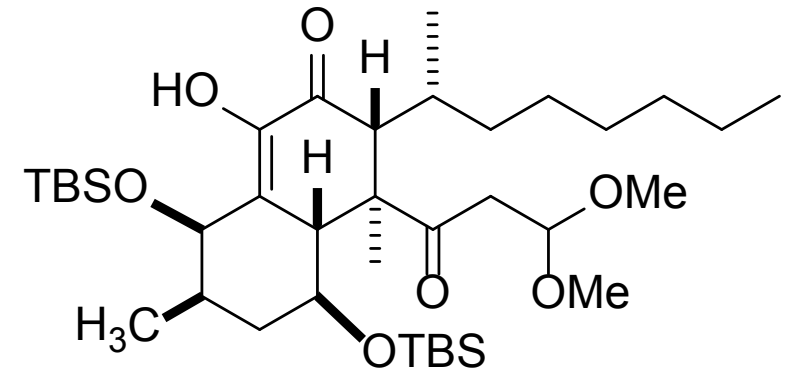

Compound 21

[Scheme 3]

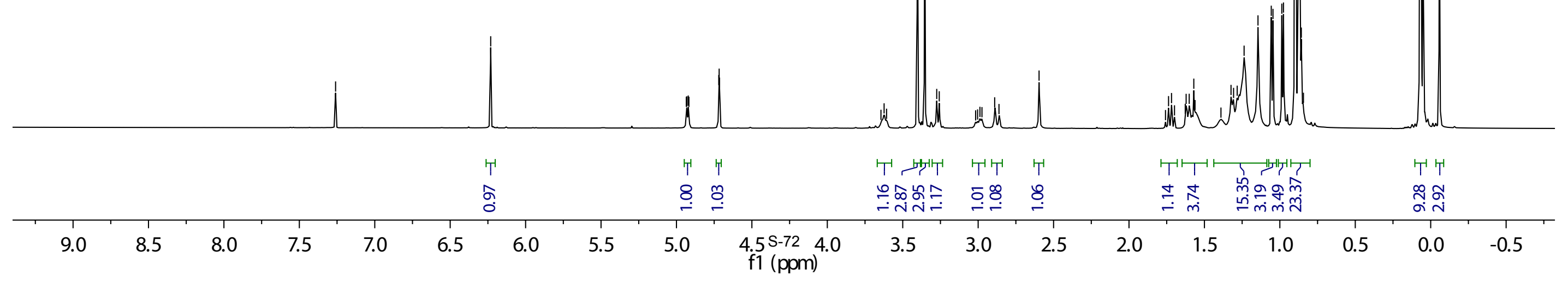


Parameters

\begin{tabular}{|lll|}
\hline \multicolumn{2}{|c|}{ Parameter } & \multicolumn{1}{c|}{ Value } \\
1 & Title & SAB-4-292-C2 \\
2 & Origin & Varian \\
3 & Spectrometer & inova \\
4 & Solvent & cdcl3 \\
5 & Temperature & 25.0 \\
6 & Pulse Sequence & s2pul \\
7 & Number of Scans & 20112 \\
8 & Receiver Gain & 60 \\
9 & Relaxation Delay & 1.5000 \\
10 & Pulse Width & 0.0000 \\
11 & Acquisition Time & 1.0428 \\
12 & Acquisition Date & $2015-12-01 T 18: 49: 10$ \\
13 & Spectrometer Frequency & 125.69 \\
14 & Spectral Width & 31421.8 \\
15 & Lowest Frequency & -1882.3 \\
16 & Nucleus & $13 \mathrm{C}$ \\
17 & Acquired Size & 32768 \\
18 & Spectral Size & 65536 \\
\hline
\end{tabular}

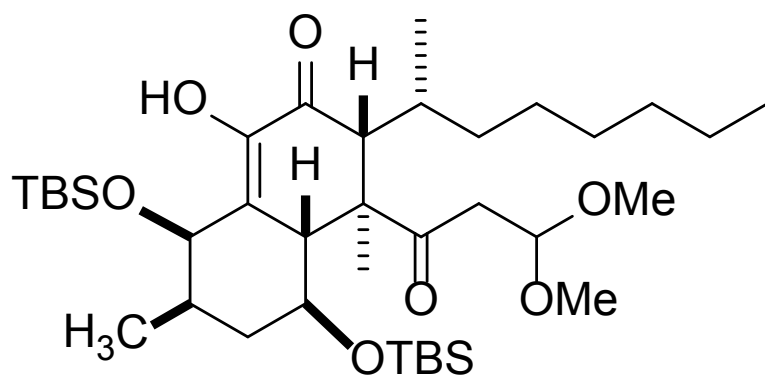

Compound 21

[Scheme 3]

90

80

$70 \quad 60$

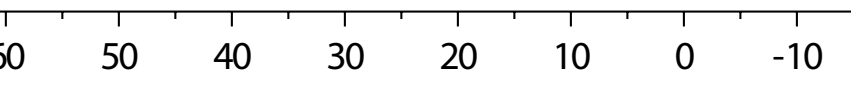




\begin{tabular}{|lll|}
\hline \multicolumn{2}{|c|}{ Parameter } & \multicolumn{1}{c|}{ Value } \\
1 & Title & SAB-4-302-2 \\
2 & Origin & Varian \\
3 & Spectrometer & inova \\
4 & Solvent & cdcl3 \\
5 & Temperature & 25.0 \\
6 & Pulse Sequence & s2pul \\
7 & Number of Scans & 32 \\
8 & Receiver Gain & 50 \\
9 & Relaxation Delay & 1.0000 \\
10 & Pulse Width & 0.0000 \\
11 & Acquisition Time & 1.7046 \\
12 & Acquisition Date & $2015-12-01 T 18: 35: 59$ \\
13 & Spectrometer Frequency & 599.78 \\
14 & Spectral Width & 9611.9 \\
15 & Lowest Frequency & -1197.7 \\
16 & Nucleus & $1 \mathrm{H}$ \\
17 & Acquired Size & 16384 \\
18 & Spectral Size & 65536 \\
\hline
\end{tabular}

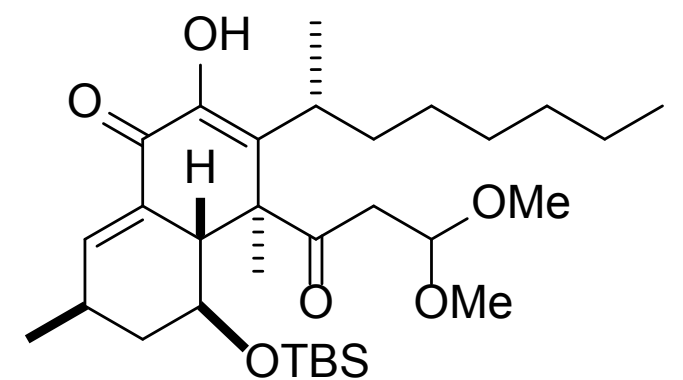

Compound 27

[Scheme 2, Step 1]

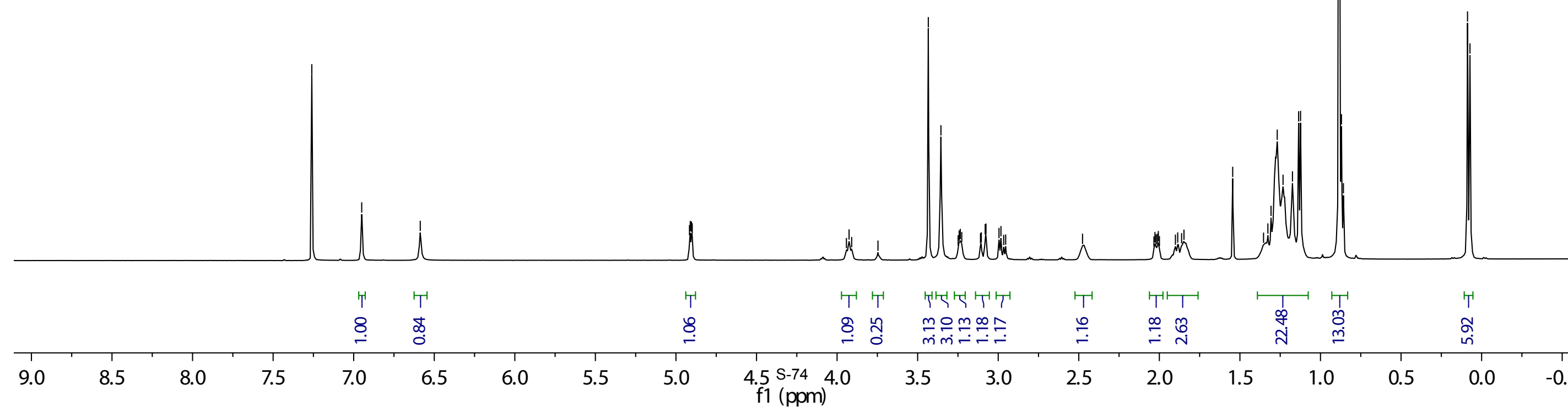




\begin{tabular}{|lll|}
\hline \multicolumn{2}{|c|}{ Parameter } & \multicolumn{1}{c|}{ Value } \\
1 & Title & SAB-4-30Z-C \\
2 & Origin & Varian \\
3 & Spectrometer & inova \\
4 & Solvent & cdcl3 \\
5 & Temperature & 24.1 \\
6 & Pulse Sequence & s2pul \\
7 & Number of Scans & 20000 \\
8 & Receiver Gain & 60 \\
9 & Relaxation Delay & 1.5000 \\
10 & Pulse Width & 0.0000 \\
11 & Acquisition Time & 1.0428 \\
12 & Acquisition Date & $2015-11-18 T 09: 17: 50$ \\
13 & Spectrometer Frequency & 125.69 \\
14 & Spectral Width & 31421.8 \\
15 & Lowest Frequency & -1867.8 \\
16 & Nucleus & $13 C$ \\
17 & Acquired Size & 32768 \\
18 & Spectral Size & 65536 \\
\hline
\end{tabular}

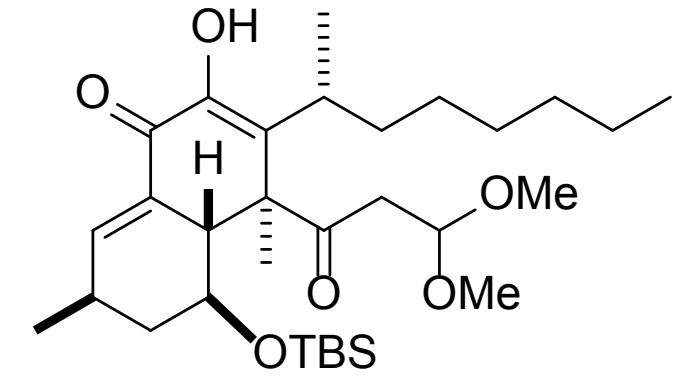

Compound 27

[Scheme 2, Step 1] 


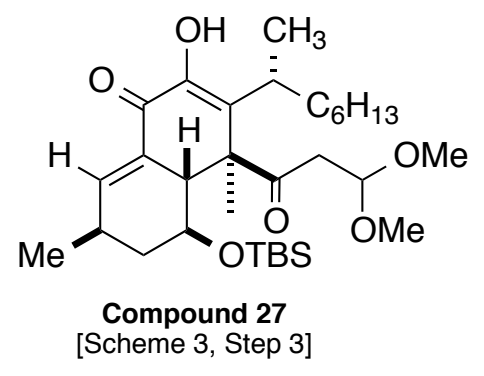

\begin{tabular}{|lll|}
\hline \multicolumn{1}{|c|}{ Parameter } & \multicolumn{1}{c|}{ Value } \\
1 & Title & III-Ick-179H_bttm_spot_COSY \\
2 & Origin & Varian \\
3 & Spectrometer & inova \\
4 & Author & jcklein \\
5 & Solvent & cdcl3 \\
6 & Temperature & 25.0 \\
7 & Pulse Sequence & gCOSY \\
8 & Number of Scans & 4 \\
9 & Receiver Gain & 60 \\
10 & Relaxation Delay & 1.0000 \\
11 & Pulse Width & 0.0000 \\
12 & Acquisition Time & 0.1499 \\
13 & Acquisition Date & $2010-09-11 \mathrm{~T} 13: 46: 35$ \\
14 & Spectrometer Frequency & $(400.11,400.11)$ \\
15 & Spectral Width & $(3416.2,3416.2)$ \\
16 & Lowest Frequency & $(-228.9,-228.9)$ \\
17 & Nucleus & $(1 \mathrm{H}, 1 \mathrm{H})$ \\
18 & Acquired Size & $(512,128)$ \\
19 & Spectral Size & $(512,512)$ \\
\hline
\end{tabular}

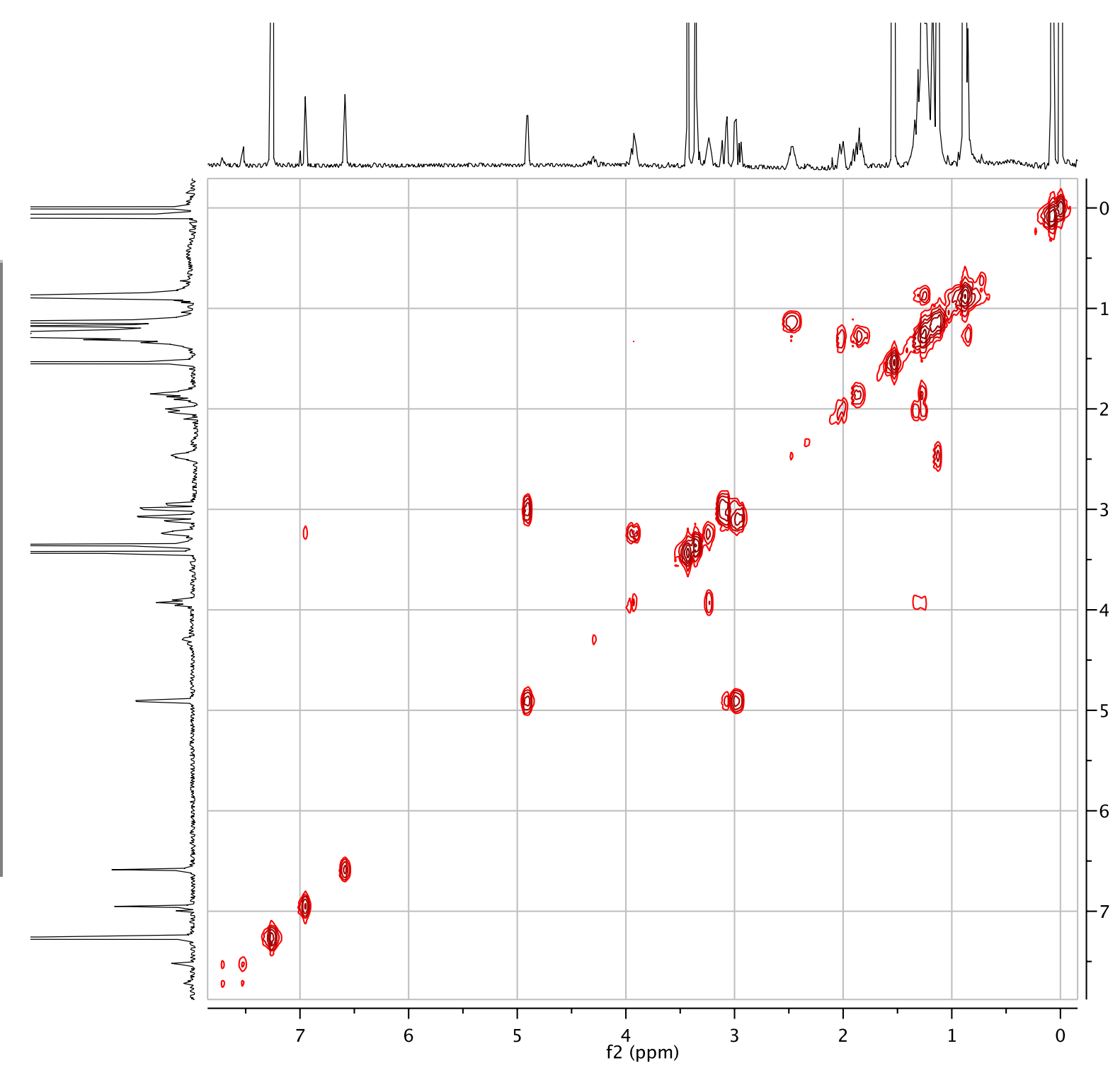




\section{Additional Computational data:}

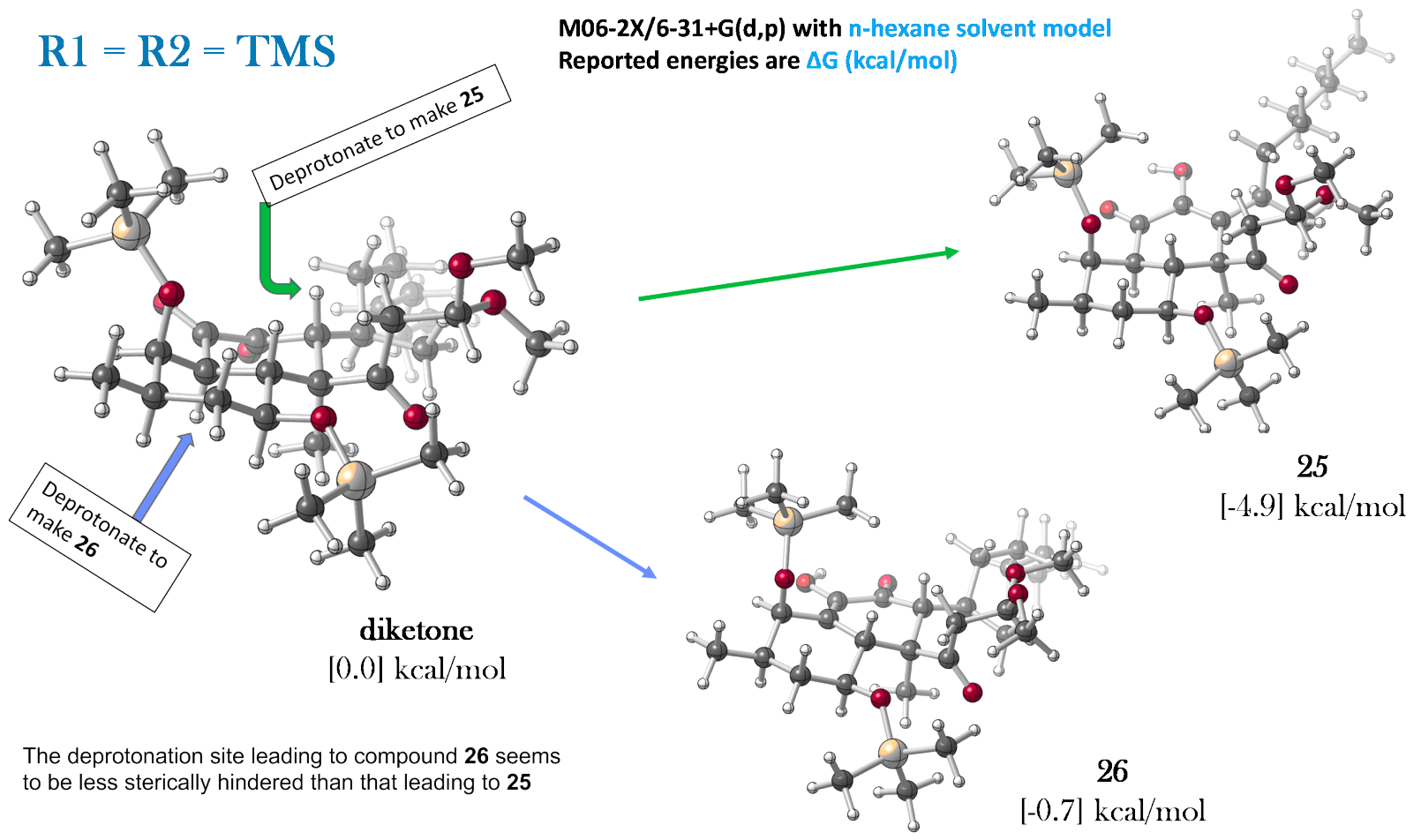

\section{Cartesian coordinates for the computed structures mentioned in the main text:}

Routecard includes: $m 062 x / 6-31+g(d, p)$ scrf=(smd, solvent=n-hexane)

1 Imaginary Frequencies: none found

Zero-point correction $=0.571920($ Hartree $/$ Particle $)$

Temperature 298.150 Kelvin. Pressure 1.00000 Atm.

Sum of electronic and thermal Free Energies = -1348.487062 hartrees $(-846189.11627562 \mathrm{kcal} / \mathrm{mol})$

$\begin{array}{lrrr}C & -3.633393 & -2.038665 & -0.181123 \\ C & -2.390346 & -1.445940 & 0.502652 \\ C & -2.193115 & 0.047600 & 0.197410 \\ C & -3.467596 & 0.800397 & 0.634424 \\ C & -4.681954 & 0.238689 & -0.094453 \\ C & -4.887578 & -1.248722 & 0.190024 \\ C & -0.864089 & 0.602790 & 0.818646 \\ C & -1.169951 & -2.238894 & 0.123842 \\ C & 0.148279 & -1.585165 & 0.177529 \\ C & 0.342274 & -0.273751 & 0.445186 \\ C & -6.124439 & -1.798259 & -0.513766 \\ C & -0.976884 & 0.682395 & 2.353067 \\ C & -0.652425 & 2.011678 & 0.237697 \\ C & -0.416624 & 2.157147 & -1.180300 \\ C & -0.221567 & 3.395331 & -1.708920 \\ C & 1.800112 & 0.199417 & 0.456324 \\ C & 2.394635 & 0.163971 & -0.975914 \\ C & 3.907250 & 0.405486 & -1.037776 \\ C & 4.739902 & -0.565777 & -0.201177\end{array}$




$\begin{array}{rrrr}\mathrm{C} & 6.242127 & -0.398390 & -0.423938 \\ \mathrm{C} & 7.090531 & -1.336055 & 0.433324 \\ \mathrm{C} & 8.587443 & -1.155578 & 0.195047 \\ \mathrm{C} & 2.122491 & 1.548274 & 1.111447 \\ \mathrm{O} & -3.478450 & -2.010310 & -1.593659 \\ \mathrm{O} & -3.424824 & 2.185401 & 0.336952 \\ \mathrm{O} & -1.226680 & -3.416765 & -0.216376 \\ \mathrm{O} & 1.188850 & -2.425438 & -0.059742 \\ \mathrm{O} & -0.703248 & 3.004278 & 0.986879 \\ \mathrm{O} & -0.222512 & 4.515548 & -1.012966 \\ \mathrm{H} & -2.515302 & -1.584639 & 1.588778 \\ \mathrm{H} & -3.749388 & -3.077448 & 0.154528 \\ \mathrm{H} & -3.613927 & 0.652435 & 1.716987 \\ \mathrm{H} & -5.563599 & 0.809330 & 0.215733 \\ \mathrm{H} & -4.550657 & 0.399793 & -1.172188 \\ \mathrm{H} & -5.017739 & -1.374575 & 1.275612 \\ \mathrm{H} & -2.123025 & 0.148824 & -0.893886 \\ \mathrm{H} & -6.262200 & -2.863269 & -0.297829 \\ \mathrm{H} & -7.023143 & -1.267342 & -0.184565 \\ \mathrm{H} & -6.032359 & -1.682193 & -1.597039 \\ \mathrm{H} & -0.037949 & 0.995655 & 2.808632 \\ \mathrm{H} & -1.735936 & 1.404026 & 2.664916 \\ \mathrm{H} & -1.232249 & -0.296849 & 2.765573 \\ \mathrm{H} & 1.893286 & 0.931633 & -1.575886 \\ \mathrm{H} & 2.173940 & -0.805988 & -1.431148 \\ \mathrm{H} & 4.215141 & 0.319110 & -2.088160 \\ \mathrm{H} & 4.142014 & 1.435975 & -0.741222 \\ \mathrm{H} & 4.524946 & -0.422547 & 0.867044 \\ \mathrm{H} & 4.444362 & -1.597688 & -0.438782 \\ \mathrm{H} & 6.474617 & -0.568818 & -1.484749 \\ \mathrm{H} & 6.526080 & 0.642250 & -0.209789 \\ \mathrm{H} & 6.805683 & -2.375142 & 0.222150 \\ \mathrm{H} & 6.861733 & -1.161113 & 1.492780 \\ \mathrm{H} & 9.181102 & -1.826618 & 0.823172 \\ \mathrm{H} & 8.845080 & -1.361012 & -0.849830 \\ \mathrm{H} & 8.897689 & -0.128611 & 0.417651 \\ \mathrm{H} & 1.893472 & 2.392307 & 0.453242 \\ \mathrm{H} & 1.609984 & 1.712303 & 2.059921 \\ \mathrm{H} & 3.195484 & 1.587827 & 1.321300 \\ \mathrm{H} & 2.325697 & -0.576691 & 1.029024 \\ \mathrm{H} & 0.803602 & -3.299534 & -0.243509 \\ \mathrm{H} & -2.851115 & 2.647481 & 0.961213 \\ \mathrm{H} & -2.891558 & -2.735445 & -1.842696 \\ \mathrm{H} & -0.407664 & 4.258638 & -0.069456 \\ \mathrm{H} & -0.399063 & 1.292233 & -1.830376 \\ \mathrm{H} & -0.042623 & 3.546095 & -2.771372 \\ & & & \\ & & & \\ & & & \end{array}$

22 Imaginary Frequencies: none found Zero-point correction $=0.572237$ (Hartree/Particle)

Temperature 298.150 Kelvin. Pressure 1.00000 Atm. Sum of electronic and thermal Free Energies = -1348.481264 hartrees $(-846185.47797264 \mathrm{kcal} / \mathrm{mol})$

$\begin{array}{lrrr}\mathrm{C} & 3.573197 & -2.048858 & 0.470276 \\ \mathrm{C} & 2.226142 & -1.428441 & 0.160452 \\ \mathrm{C} & 2.177284 & 0.069480 & -0.056555 \\ \mathrm{C} & 3.310752 & 0.475055 & -1.037011 \\ \mathrm{C} & 4.657552 & -0.070562 & -0.586666 \\ \mathrm{C} & 4.651640 & -1.595443 & -0.511792 \\ \mathrm{C} & 0.768212 & 0.609240 & -0.509144 \\ \mathrm{C} & 1.118169 & -2.184778 & 0.162986\end{array}$




\begin{tabular}{|c|c|c|c|}
\hline$C$ & & & \\
\hline c & -0.238411 & -1.592259 & 0.104953 \\
\hline$c$ & $\begin{array}{r}-0.359829 \\
6.020196\end{array}$ & $\begin{array}{l}-0.0894 \angle 0 \\
-2.159445\end{array}$ & $\begin{array}{r}0.314794 \\
-0.141963\end{array}$ \\
\hline c & 0.525031 & 0.368340 & -2.005486 \\
\hline C & 0.770707 & 2.117151 & -0.198940 \\
\hline C & 0.818818 & 2.570049 & 1.175500 \\
\hline C & 0.837911 & 3.903233 & 1.449069 \\
\hline C & -1.842301 & 0.334599 & 0.107862 \\
\hline C & -2.617062 & -0.016385 & 1.394014 \\
\hline C & -4.135093 & 0.181069 & 1.326953 \\
\hline C & -4.815402 & -0.578562 & 0.187812 \\
\hline C & -6.339217 & -0.480033 & 0.238275 \\
\hline C & -7.032482 & -1.217264 & -0.906590 \\
\hline C & -8.553167 & -1.096722 & -0.849607 \\
\hline C & -2.113722 & 1.791002 & -0.277150 \\
\hline 0 & 3.981958 & -1.630154 & 1.776026 \\
\hline 0 & 3.452873 & 1.881718 & -1.122064 \\
\hline 0 & 1.140886 & -3.536079 & 0.284155 \\
\hline 0 & -1.189549 & -2.347766 & -0.018460 \\
\hline 0 & 0.762336 & 2.938658 & -1.134402 \\
\hline 0 & 0.804878 & 4.857772 & 0.541967 \\
\hline $\mathrm{H}$ & 3.485025 & -3.140828 & 0.441056 \\
\hline $\mathrm{H}$ & 3.087038 & 0.053595 & -2.028592 \\
\hline $\mathrm{H}$ & 5.416783 & 0.275227 & -1.296241 \\
\hline $\mathrm{H}$ & 4.906782 & 0.352122 & 0.394807 \\
\hline $\mathrm{H}$ & 4.357742 & -1.992024 & -1.494929 \\
\hline $\mathrm{H}$ & 2.442533 & 0.546091 & 0.899947 \\
\hline $\mathrm{H}$ & -0.108905 & 0.047048 & 1.379272 \\
\hline $\mathrm{H}$ & 5.991871 & -3.252195 & -0.072483 \\
\hline $\mathrm{H}$ & 6.763421 & -1.887588 & -0.897986 \\
\hline $\mathrm{H}$ & 6.351164 & -1.767363 & 0.823471 \\
\hline H & -0.507538 & 0.601812 & -2.275993 \\
\hline $\mathrm{H}$ & 1.163601 & 0.988981 & -2.636426 \\
\hline $\mathrm{H}$ & 0.710853 & -0.679949 & -2.259892 \\
\hline $\mathrm{H}$ & -2.214903 & 0.605221 & 2.208124 \\
\hline $\mathrm{H}$ & -2.413703 & -1.060061 & 1.658012 \\
\hline $\mathrm{H}$ & -4.561823 & -0.155163 & 2.280937 \\
\hline $\mathrm{H}$ & -4.378516 & 1.248718 & 1.249007 \\
\hline $\mathrm{H}$ & -4.467654 & -0.190954 & -0.779658 \\
\hline $\mathrm{H}$ & -4.511590 & -1.634412 & 0.221874 \\
\hline $\mathrm{H}$ & -6.699392 & -0.880181 & 1.196726 \\
\hline $\mathrm{H}$ & -6.634635 & 0.579024 & 0.217105 \\
\hline $\mathrm{H}$ & -6.744897 & -2.276358 & -0.879763 \\
\hline $\mathrm{H}$ & -6.667185 & -0.822199 & -1.863496 \\
\hline $\mathrm{H}$ & -9.031886 & -1.629604 & -1.676740 \\
\hline $\mathrm{H}$ & -8.945115 & -1.511316 & 0.085739 \\
\hline $\mathrm{H}$ & -8.865477 & -0.047923 & -0.902824 \\
\hline $\mathrm{H}$ & -1.860833 & 2.485275 & 0.531865 \\
\hline $\mathrm{H}$ & -1.583352 & 2.106985 & -1.176648 \\
\hline $\mathrm{H}$ & -3.181241 & 1.909086 & -0.485957 \\
\hline $\mathrm{H}$ & -2.228932 & -0.295546 & -0.701941 \\
\hline $\mathrm{H}$ & 0.218930 & -3.837740 & 0.219018 \\
\hline $\mathrm{H}$ & 3.395665 & -2.037802 & 2.424659 \\
\hline$H$ & 2.722153 & 2.269177 & -1.620958 \\
\hline $\mathrm{H}$ & 0.783866 & 4.401408 & -0.342522 \\
\hline $\mathrm{H}$ & 0.851027 & 1.872913 & 2.001500 \\
\hline $\mathrm{H}$ & 0.878520 & 4.277098 & 2.469903 \\
\hline
\end{tabular}

23 Imaginary Frequencies: none found Zero-point correction $=0.656143$ (Hartree/Particle)

Temperature 298.150 Kelvin. Pressure 1.00000 Atm. Sum of electronic and thermal Free Energies = 
-1503.384254 hartrees (-943388.65322754 kcal/mo1)

\begin{tabular}{|c|c|c|c|}
\hline & & & \\
\hline C & -3.821132 & -2.232682 & -0.554563 \\
\hline C & -2.578722 & -1.863714 & 0.274606 \\
\hline C & -2.330000 & -0.349097 & 0.334906 \\
\hline C & -3.596385 & 0.324921 & 0.900232 \\
\hline C & -4.806507 & -0.003879 & 0.035747 \\
\hline C & -5.062779 & -1.509706 & -0.036996 \\
\hline C & -1.014151 & 0.011758 & 1.106871 \\
\hline C & -1.372125 & -2.569142 & -0.279535 \\
\hline C & -0.043033 & -1.991872 & -0.028642 \\
\hline C & 0.181583 & -0.813369 & 0.597294 \\
\hline C & -6.291304 & -1.837267 & -0.880271 \\
\hline C & -1.184989 & -0.231463 & 2.616681 \\
\hline C & -0.750544 & 1.518959 & 0.865279 \\
\hline C & -0.669098 & 2.023358 & -0.559948 \\
\hline C & 0.052266 & 3.356581 & -0.654178 \\
\hline C & 1.790675 & 4.134056 & 0.790775 \\
\hline C & 0.618045 & 4.979292 & -2.257642 \\
\hline C & 1.658660 & -0.456561 & 0.786179 \\
\hline C & 2.270151 & 0.003920 & -0.562692 \\
\hline C & 3.802025 & 0.077203 & -0.571984 \\
\hline C & 4.501884 & -1.209541 & -0.133854 \\
\hline C & 6.004578 & -1.194407 & -0.409870 \\
\hline C & 6.718268 & -2.457737 & 0.067658 \\
\hline C & 8.215529 & -2.440721 & -0.229192 \\
\hline C & 2.039463 & 0.511503 & 1.909400 \\
\hline 0 & -3.627208 & -1.872638 & -1.915295 \\
\hline 0 & -3.487482 & 1.741446 & 0.938505 \\
\hline 0 & -1.450484 & -3.616586 & -0.914494 \\
\hline 0 & 0.983736 & -2.768848 & -0.463126 \\
\hline 0 & -0.675827 & 2.292737 & 1.801232 \\
\hline 0 & 1.379882 & 3.212118 & -0.203979 \\
\hline 0 & 0.020917 & 3.723593 & -2.000821 \\
\hline $\mathrm{H}$ & -2.723517 & -2.255843 & 1.294271 \\
\hline $\mathrm{H}$ & -3.977076 & -3.316719 & -0.478727 \\
\hline $\mathrm{H}$ & -3.783658 & -0.062248 & 1.914119 \\
\hline $\mathrm{H}$ & -5.678675 & 0.508989 & 0.455277 \\
\hline $\mathrm{H}$ & -4.643756 & 0.397259 & -0.973002 \\
\hline $\mathrm{H}$ & -5.230399 & -1.879939 & 0.985950 \\
\hline $\mathrm{H}$ & -2.217581 & -0.012643 & -0.704448 \\
\hline $\mathrm{H}$ & -6.461790 & -2.918294 & -0.926275 \\
\hline $\mathrm{H}$ & -7.186766 & -1.371571 & -0.457028 \\
\hline $\mathrm{H}$ & -6.162004 & -1.471835 & -1.902483 \\
\hline $\mathrm{H}$ & -0.271452 & 0.004027 & 3.159388 \\
\hline $\mathrm{H}$ & -1.969568 & 0.399984 & 3.041928 \\
\hline $\mathrm{H}$ & -1.434212 & -1.278949 & 2.806999 \\
\hline $\mathrm{H}$ & -1.698953 & 2.157780 & -0.910903 \\
\hline $\mathrm{H}$ & -0.194217 & 1.292667 & -1.221889 \\
\hline $\mathrm{H}$ & -0.451886 & 4.122951 & -0.042391 \\
\hline $\mathrm{H}$ & 2.840643 & 3.919699 & 1.001995 \\
\hline $\mathrm{H}$ & 1.706341 & 5.171200 & 0.439884 \\
\hline $\mathrm{H}$ & 1.201853 & 4.012909 & 1.707955 \\
\hline $\mathrm{H}$ & 1.693244 & 4.957774 & -2.046537 \\
\hline $\mathrm{H}$ & 0.465462 & 5.192477 & -3.316802 \\
\hline $\mathrm{H}$ & 0.142126 & 5.771336 & -1.661179 \\
\hline $\mathrm{H}$ & 1.882217 & 0.995898 & -0.809098 \\
\hline $\mathrm{H}$ & 1.948454 & -0.693715 & -1.344097 \\
\hline $\mathrm{H}$ & 4.112366 & 0.319924 & -1.596938 \\
\hline $\mathrm{H}$ & 4.147606 & 0.914480 & 0.048828 \\
\hline $\mathrm{H}$ & 4.343482 & -1.376979 & 0.940841 \\
\hline $\mathrm{H}$ & 4.041714 & -2.065704 & -0.647686 \\
\hline $\mathrm{H}$ & 6.173929 & -1.068637 & -1.488621 \\
\hline H & & -0.317424 & \\
\hline
\end{tabular}




$\begin{array}{rrrr}\mathrm{H} & 6.257734 & -3.332690 & -0.409516 \\ \mathrm{H} & 6.558524 & -2.575550 & 1.147482 \\ \mathrm{H} & 8.706338 & -3.352900 & 0.123703 \\ \mathrm{H} & 8.401327 & -2.356716 & -1.305610 \\ \mathrm{H} & 8.702812 & -1.589247 & 0.258662 \\ \mathrm{H} & 1.697162 & 1.531368 & 1.721496 \\ \mathrm{H} & 1.674919 & 0.188527 & 2.886758 \\ \mathrm{H} & 3.131146 & 0.543865 & 1.980378 \\ \mathrm{H} & 2.131054 & -1.418157 & 1.021433 \\ \mathrm{H} & 0.584227 & -3.553580 & -0.875598 \\ \mathrm{H} & -3.009908 & 2.021370 & 1.729408 \\ \mathrm{H} & -3.048020 & -2.532074 & -2.318024\end{array}$

24 Imaginary Frequencies: none found

Zero-point correction $=0.656902$ (Hartree/Particle)

Temperature 298.150 Kelvin. Pressure 1.00000 Atm. Sum of electronic and thermal Free Energies = -1503.375174 hartrees $(-943382.95543674 \mathrm{kcal} / \mathrm{mol})$

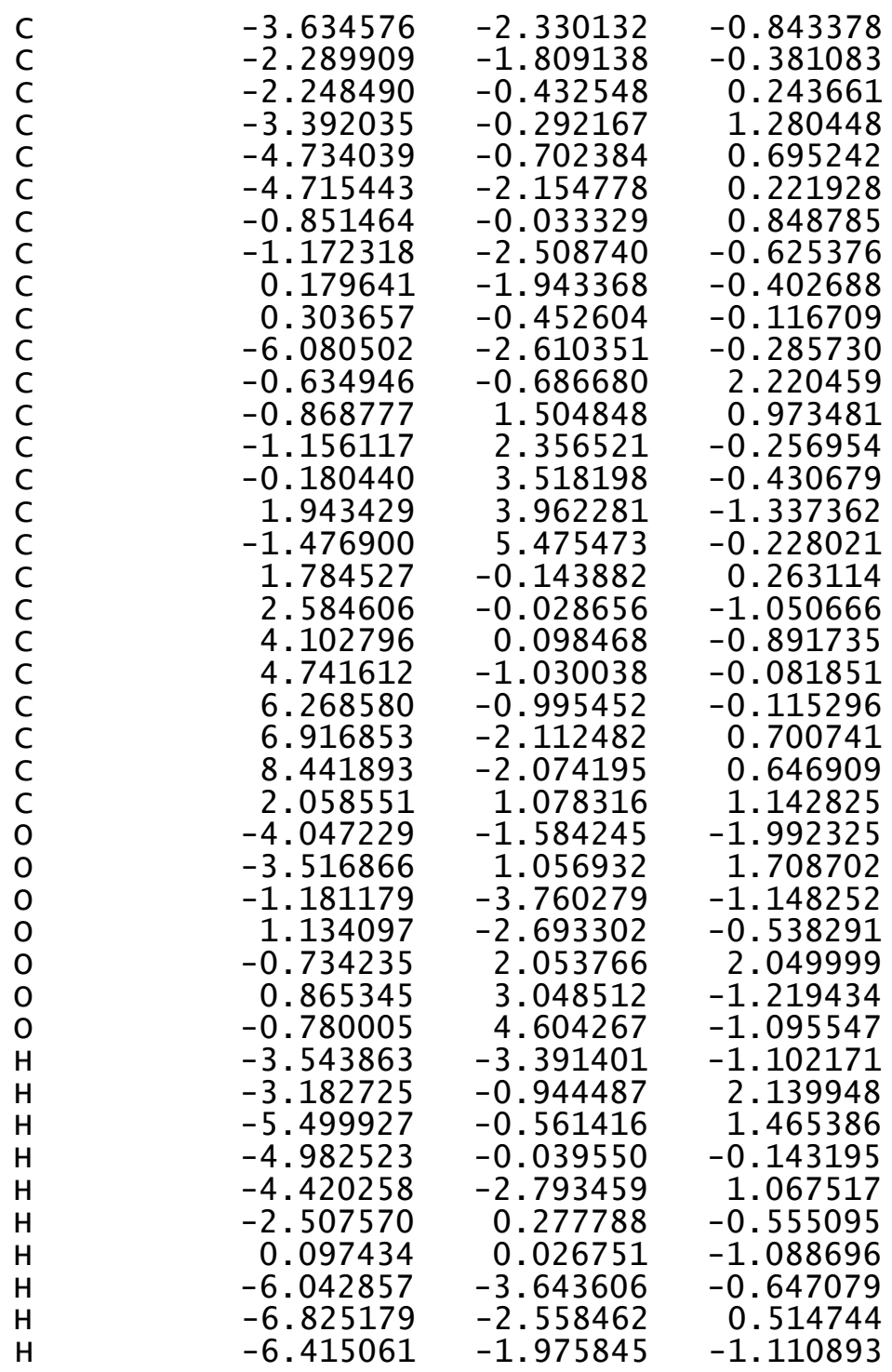




$\begin{array}{rrrr}\mathrm{H} & 0.398670 & -0.575498 & 2.552309 \\ \mathrm{H} & -1.261322 & -0.238533 & 2.995082 \\ \mathrm{H} & -0.858466 & -1.757389 & 2.175870 \\ \mathrm{H} & -2.164540 & 2.753663 & -0.090472 \\ \mathrm{H} & -1.170626 & 1.794128 & -1.192331 \\ \mathrm{H} & 0.198143 & 3.862885 & 0.549662 \\ \mathrm{H} & 2.740328 & 3.445203 & -1.875264 \\ \mathrm{H} & 1.643819 & 4.854548 & -1.895040 \\ \mathrm{H} & 2.312677 & 4.260990 & -0.345932 \\ \mathrm{H} & -0.798328 & 5.925412 & 0.509340 \\ \mathrm{H} & -1.909568 & 6.262435 & -0.847250 \\ \mathrm{H} & -2.287015 & 4.961907 & 0.307637 \\ \mathrm{H} & 2.202715 & 0.847547 & -1.593065 \\ \mathrm{H} & 2.375697 & -0.909637 & -1.668398 \\ \mathrm{H} & 4.547330 & 0.107839 & -1.895919 \\ \mathrm{H} & 4.363074 & 1.064991 & -0.439708 \\ \mathrm{H} & 4.407115 & -0.976144 & 0.963535 \\ \mathrm{H} & 4.386448 & -1.996580 & -0.466573 \\ \mathrm{H} & 6.610456 & -1.065025 & -1.157793 \\ \mathrm{H} & 6.620267 & -0.022894 & 0.258758 \\ \mathrm{H} & 6.558750 & -3.081770 & 0.330264 \\ \mathrm{H} & 6.582390 & -2.038038 & 1.743664 \\ \mathrm{H} & 8.887153 & -2.881739 & 1.235995 \\ \mathrm{H} & 8.800476 & -2.176140 & -0.383275 \\ \mathrm{H} & 8.824985 & -1.125166 & 1.038041 \\ \mathrm{H} & 1.816389 & 2.008395 & 0.621555 \\ \mathrm{H} & 1.521757 & 1.065222 & 2.091413 \\ \mathrm{H} & 3.126421 & 1.103320 & 1.381436 \\ \mathrm{H} & 2.148263 & -1.024553 & 0.805957 \\ \mathrm{H} & -0.253731 & -4.049846 & -1.192130 \\ \mathrm{H} & -3.437202 & -1.774232 & -2.715399 \\ \mathrm{H} & -2.870574 & 1.254101 & 2.398367 \\ & & & \\ & & & \end{array}$

25TMS-TMS Imaginary Frequencies: none found Zero-point correction $=0.859882($ Hartree/Particle)

Temperature 298.150 Kelvin. Pressure 1.00000 Atm. Sum of electronic and thermal Free Energies = -2320.441503 hartrees $(-1456100.24754753 \mathrm{kcal} / \mathrm{mol})$

$\begin{array}{lrrr}\text { Si } & -3.359432 & -3.198591 & -0.799338 \\ \text { Si } & -1.881038 & 3.856520 & 1.581985 \\ \text { C } & -2.736228 & 2.445769 & -0.683082 \\ \text { C } & -1.598119 & 1.660519 & -1.358382 \\ \text { C } & -1.437713 & 0.278334 & -0.717904 \\ \text { C } & -2.755658 & -0.489708 & -0.925830 \\ \text { C } & -3.931011 & 0.279967 & -0.319530 \\ \text { C } & -4.061948 & 1.702544 & -0.870197 \\ \text { C } & -0.165596 & -0.485525 & -1.206304 \\ \text { C } & -0.322546 & 2.455034 & -1.317639 \\ \text { C } & 0.952107 & 1.747125 & -1.126863 \\ \text { C } & 1.086798 & 0.400818 & -1.065623 \\ \text { C } & -5.215358 & 2.455253 & -0.214598 \\ \text { C } & -0.319211 & -0.905782 & -2.677492 \\ \text { C } & -0.007405 & -1.754174 & -0.330475 \\ \text { C } & -0.026111 & -1.582693 & 1.176803 \\ \text { C } & 0.565617 & -2.775424 & 1.901957 \\ \text { C } & 2.355542 & -4.170415 & 1.159602 \\ \text { C } & 0.843302 & -3.595608 & 4.087296 \\ \text { C } & 2.532028 & -0.096626 & -0.975597 \\ \text { C } & 3.084885 & 0.134760 & 0.455059 \\ \text { C } & 4.589589 & -0.119227 & 0.599190 \\ \text { C } & 5.471605 & 0.714009 & -0.329855\end{array}$




\begin{tabular}{|c|c|c|c|}
\hline$C$ & רזברת & תרדרמזז0 & \\
\hline 2 & 6.962552 & 0.502726 & -0.072104 \\
\hline & 7.860923 & 1.330633 & -0.989016 \\
\hline & 9.346321 & 1.086638 & -0.734510 \\
\hline C & 2.853153 & -1.521120 & -1.439009 \\
\hline C & -0.042054 & 3.635875 & 1.884654 \\
\hline C & -2.795616 & 3.772113 & 3.214011 \\
\hline C & -2.240319 & 5.465142 & 0.692818 \\
\hline C & -2.662405 & -4.467665 & 0.380149 \\
\hline C & -5.228406 & -3.084670 & -0.656071 \\
\hline C & -2.912286 & -3.613286 & -2.572187 \\
\hline 0 & -2.470133 & 2.555588 & 0.700447 \\
\hline 0 & -2.646075 & -1.758999 & -0.313390 \\
\hline 0 & -0.303706 & 3.672915 & -1.466715 \\
\hline 0 & 2.034708 & 569813 & -1.102610 \\
\hline 0 & 0.116660 & -2.852281 & -0.834891 \\
\hline 0 & 1.936686 & -2.889284 & 1.597368 \\
\hline 0 & 0.375782 & -2.543336 & 3.267999 \\
\hline $\mathrm{H}$ & -1.854311 & 556894 & -2.424920 \\
\hline $\mathrm{H}$ & -2.797551 & 3.441296 & -1.143986 \\
\hline $\mathrm{H}$ & -2.928470 & -0.603660 & -2.010963 \\
\hline $\mathrm{H}$ & -4.855817 & -0.278637 & -0.503949 \\
\hline $\mathrm{H}$ & -3.784887 & 0.322504 & 0.767280 \\
\hline $\mathrm{H}$ & -4.244076 & 1.649182 & -1.954197 \\
\hline $\mathrm{H}$ & -1.329538 & 0.453521 & 0.359745 \\
\hline $\mathrm{H}$ & -5.313760 & 3.465732 & -0.626364 \\
\hline $\mathrm{H}$ & -6.164995 & 1.934046 & -0.372106 \\
\hline $\mathrm{H}$ & -5.047468 & 2.540595 & 0.863747 \\
\hline $\mathrm{H}$ & 0.576932 & -1.401462 & -3.047839 \\
\hline $\mathrm{H}$ & -1.144813 & -1.610628 & -2.794515 \\
\hline $\mathrm{H}$ & -0.503394 & -0.028712 & -3.304885 \\
\hline $\mathrm{H}$ & -1.073745 & -1.475167 & 1.476422 \\
\hline $\mathrm{H}$ & 0.494580 & -0.667313 & 1.478578 \\
\hline $\mathrm{H}$ & 0.056193 & -3.707750 & 1.606868 \\
\hline $\mathrm{H}$ & 3.430868 & -4.105621 & 0.978406 \\
\hline $\mathrm{H}$ & 2.173456 & -4.940157 & 1.921385 \\
\hline $\mathrm{H}$ & 1.846363 & -4.454176 & 0.230576 \\
\hline $\mathrm{H}$ & 1.932393 & -3.698874 & 4. 019601 \\
\hline $\mathrm{H}$ & 0.572238 & -3.345610 & 5.114367 \\
\hline $\mathrm{H}$ & 0.366870 & -4.548532 & 3.812963 \\
\hline $\mathrm{H}$ & 2.565095 & -0.537798 & 1.144478 \\
\hline $\mathrm{H}$ & 2.864796 & 1.166080 & 0.753882 \\
\hline $\mathrm{H}$ & 4.866332 & 0.100024 & 1.638886 \\
\hline $\mathrm{H}$ & 4.806883 & -1.185495 & 0.450577 \\
\hline H & 5.257566 & 0.466391 & -1.378788 \\
\hline $\mathrm{H}$ & 5.222836 & 1.778161 & -0.209572 \\
\hline $\mathrm{H}$ & 7.190108 & 0.748219 & 0.975049 \\
\hline $\mathrm{H}$ & 7.203137 & -0.563258 & -0.197067 \\
\hline $\mathrm{H}$ & 7.632238 & 2.395467 & -0.850772 \\
\hline H & 7.6226 & 1.096451 & -2.034901 \\
\hline $\mathrm{H}$ & 9.974407 & 1.698651 & -1.388996 \\
\hline H & 9.610524 & 1.326375 & 0.301473 \\
\hline $\mathrm{H}$ & 9.605709 & 0.036358 & -0.908376 \\
\hline H & 2.560100 & -2.267746 & -0.698753 \\
\hline H & 2.392769 & -1.777703 & -2.394283 \\
\hline H & 3.936150 & -1.601164 & -1.576640 \\
\hline $\mathrm{H}$ & 3.083052 & 0.587184 & -1.63355 \\
\hline H & 0.189607 & 2.591230 & 2.12554 \\
\hline $\mathrm{H}$ & 0.556204 & 3.924857 & 1.01543 \\
\hline H & 0.287070 & 4.248180 & 2.732364 \\
\hline $\mathrm{H}$ & -2.434702 & 4.534756 & 3.91307 \\
\hline H & -3.870652 & 3.927620 & 3.072723 \\
\hline $\mathrm{H}$ & -2.657858 & 2.793712 & 3.687026 \\
\hline H & -1.921368 & 6.309921 & 1.314764 \\
\hline H & & 528299 & -0.2576 \\
\hline
\end{tabular}




$\begin{array}{rrrr}\mathrm{H} & -3.311481 & 5.588984 & 0.497268 \\ \mathrm{H} & -2.780872 & -4.145724 & 1.420717 \\ \mathrm{H} & -3.167335 & -5.433443 & 0.267080 \\ \mathrm{H} & -1.596385 & -4.615569 & 0.177742 \\ \mathrm{H} & -5.656032 & -2.401478 & -1.398552 \\ \mathrm{H} & -5.676906 & -4.070747 & -0.826785 \\ \mathrm{H} & -5.538623 & -2.746745 & 0.338450 \\ \mathrm{H} & -3.205942 & -2.826540 & -3.276682 \\ \mathrm{H} & -1.837531 & -3.793299 & -2.676420 \\ \mathrm{H} & -3.438258 & -4.526638 & -2.875697 \\ \mathrm{H} & 1.697278 & 3.470594 & -1.247630\end{array}$

26TMS-TMS Imaginary Frequencies: none found Zero-point correction $=0.860248($ Hartree/Particle)

Temperature 298.150 Kelvin. Pressure 1.00000 Atm. Sum of electronic and thermal Free Energies = -2320.434841 hartrees $(-1456096.06707591 \mathrm{kcal} / \mathrm{mol})$

$\begin{array}{lrrr}\mathrm{Si} & -3.241012 & -3.062219 & -1.473889 \\ \mathrm{Si} & -2.198422 & 3.623969 & 1.774776 \\ \mathrm{C} & -2.597327 & 2.533887 & -0.716048 \\ \mathrm{C} & -1.304035 & 1.758111 & -0.843945 \\ \mathrm{C} & -1.345739 & 0.281375 & -0.532079 \\ \mathrm{C} & -2.559114 & -0.379470 & -1.225446 \\ \mathrm{C} & -3.848326 & 0.398541 & -0.979128 \\ \mathrm{C} & -3.744221 & 1.845882 & -1.457169 \\ \mathrm{C} & -0.005970 & -0.485183 & -0.824290 \\ \mathrm{C} & -0.150087 & 2.393353 & -1.083058 \\ \mathrm{C} & 1.162623 & 1.728849 & -0.916208 \\ \mathrm{C} & 1.200291 & 0.346543 & -0.274509 \\ \mathrm{C} & -5.055896 & 2.602669 & -1.272261 \\ \mathrm{C} & 0.179346 & -0.736721 & -2.324532 \\ \mathrm{C} & -0.080308 & -1.828766 & -0.065264 \\ \mathrm{C} & -0.421659 & -1.832547 & 1.422139 \\ \mathrm{C} & 0.504575 & -2.717746 & 2.248238 \\ \mathrm{C} & 2.630306 & -2.644080 & 3.255937 \\ \mathrm{C} & -0.943840 & -4.309676 & 3.221818 \\ \mathrm{C} & 2.660790 & -0.194948 & -0.379644 \\ \mathrm{C} & 3.463081 & 0.430184 & 0.780938 \\ \mathrm{C} & 4.971700 & 0.164026 & 0.763284 \\ \mathrm{C} & 5.669287 & 0.578914 & -0.532051 \\ \mathrm{C} & 7.190202 & 0.461934 & -0.447798 \\ \mathrm{C} & 7.899006 & 0.815069 & -1.754166 \\ \mathrm{C} & 9.417378 & 0.693538 & -1.653363 \\ \mathrm{C} & 2.877805 & -1.709429 & -0.404014 \\ \mathrm{C} & -0.488403 & 3.006115 & 2.254463 \\ \mathrm{C} & -3.328609 & 3.578517 & 3.263773 \\ \mathrm{C} & -2.074480 & 5.344613 & 1.044388 \\ \mathrm{C} & -2.958729 & -4.426594 & -0.229620 \\ \mathrm{C} & -5.066521 & -2.880831 & -1.874788 \\ \mathrm{C} & -2.301605 & -3.402298 & -3.058838 \\ \mathrm{O} & -2.941094 & 2.601912 & 0.664868 \\ \mathrm{O} & -2.667561 & -1.687983 & -0.696659 \\ \mathrm{O} & -0.078984 & 3.715454 & -1.385551 \\ \mathrm{O} & 2.158684 & 2.367960 & -1.221418 \\ \mathrm{O} & 0.098071 & -2.888173 & -0.630982 \\ \mathrm{O} & 1.585253 & -1.925356 & 2.622937 \\ \mathrm{O} & -0.118785 & -3.182390 & 3.424042 \\ \mathrm{H} & -2.456126 & 3.545784 & -1.117696 \\ \mathrm{H} & -2.375428 & -0.418326 & -2.311718 \\ \mathrm{H} & -4.671426 & -0.109958 & -1.493689 \\ & & & \end{array}$




$\begin{array}{rrr}-4.070869 & 0.380907 & 0.095102 \\ -3.474380 & 1.850009 & -2.523923 \\ -1.569982 & 0.205455 & 0.542616 \\ 0.988014 & 0.539543 & 0.791777 \\ -4.961534 & 3.644770 & -1.597113 \\ -5.858241 & 2.138558 & -1.854411 \\ -5.349084 & 2.601173 & -0.218377 \\ 1.191777 & -1.081778 & -2.544137 \\ -0.505983 & -1.50451 & -2.684153 \\ 0.006213 & 0.181851 & -2.894908 \\ -1.446812 & -2.218062 & 1.471676 \\ -0.416228 & -0.840570 & 1.879693 \\ 0.845896 & -3.588450 & 1.657917 \\ 3.447274 & -1.937931 & 3.416846 \\ 2.302667 & -3.050075 & 4.217654 \\ 2.984178 & -3.464589 & 2.615552 \\ -0.392523 & -5.125950 & 2.734430 \\ -1.277442 & -4.641291 & 4.206508 \\ -1.826640 & -4.069813 & 2.614567 \\ 3.040007 & 0.043893 & 1.718794 \\ 3.302851 & 1.514829 & 0.777510 \\ 5.423367 & 0.714485 & 1.599423 \\ 5.175362 & -0.897087 & 0.959651 \\ 5.312244 & -0.042228 & -1.365108 \\ 5.391365 & 1.612908 & -0.780601 \\ 7.561208 & 1.115967 & 0.354227 \\ 7.459418 & -0.564172 & -0.157540 \\ 7.628964 & 1.838460 & -2.045600 \\ 7.529190 & 0.158412 & -2.552530 \\ 9.906330 & 0.949187 & -2.598400 \\ 9.813834 & 1.361772 & -0.880968 \\ 9.712214 & -0.328442 & -1.390692 \\ 2.592355 & -2.171320 & 0.544849 \\ 2.339914 & -2.213761 & -1.206542 \\ 3.943568 & -1.905928 & -0.558986 \\ 3.061791 & 0.202396 & -1.319306 \\ -0.488572 & 1.923320 & 2.427786 \\ 0.266332 & 3.239408 & 1.495953 \\ -0.170406 & 3.483840 & 3.189120 \\ -2.958457 & 4.236689 & 4.057704 \\ -4.339628 & 3.903881 & 2.997734 \\ -3.398907 & 2.565130 & 3.673133 \\ -1.684402 & 6.048553 & 1.788691 \\ -1.396674 & 5.363307 & 0.184094 \\ -3.055043 & 5.710829 & 0.719807 \\ -3.525660 & -4.245477 & 0.689978 \\ -3.274630 & -5.396248 & -0.630573 \\ -1.892316 & -4.491949 & 0.014388 \\ -5.244363 & -2.153549 & -2.674793 \\ -5.467366 & -3.842440 & -2.217858 \\ -5.644923 & -2.573840 & -0.996827 \\ -2.358402 & -2.576140 & -3.776757 \\ -1.248525 & -3.613492 & -2.847728 \\ -2.730837 & -4.284000 & -3.550540 \\ 0.863758 & 3.917217 & -1.516583\end{array}$

\section{TBS-TMS}

$\mathrm{HF}=-2439.0868687$ hartrees $(-1530551.40097794 \mathrm{kcal} / \mathrm{mol})$

$\begin{array}{lrrr}\text { Si } & 2.293432 & 4.184622 & -0.587810 \\ \text { Si } & 2.473397 & -3.320960 & 0.536817 \\ \text { C } & 2.808965 & -1.401472 & -1.476900 \\ \text { C } & 1.478161 & -0.772384 & -1.925394\end{array}$




\begin{tabular}{|c|c|c|c|}
\hline$C$ & & & \\
\hline c & 1.111747 & 0.417055 & -1.032447 \\
\hline c & 3.583202 & 0.875316 & -0.793401 \\
\hline C & 3.937579 & -0.374326 & -1.603905 \\
\hline C & -0.327360 & 0.965845 & -1.293405 \\
\hline C & 0.394309 & -1.813739 & -1.954480 \\
\hline C & -0.970061 & -1.433426 & -1.559353 \\
\hline C & -1.360766 & -0.175818 & -1.242798 \\
\hline C & 5.274978 & -0.965539 & -1.169665 \\
\hline C & -0.403676 & 1.650047 & -2.668415 \\
\hline C & -0.646307 & 2.006902 & -0.190660 \\
\hline C & -0.447387 & 1.592589 & 1.255167 \\
\hline C & -1.188991 & 2.494260 & 2.222436 \\
\hline C & -3.282346 & 3.584021 & 1.860209 \\
\hline C & -1.408533 & 2.858316 & 4.534314 \\
\hline C & -2.859890 & -0.017276 & -0.974959 \\
\hline C & -3.214215 & -0.597152 & 0.419374 \\
\hline C & -4.718115 & -0.694684 & 0.699001 \\
\hline C & -5.504911 & -1.527304 & -0.312461 \\
\hline C & -6.975903 & -1.682131 & 0.069912 \\
\hline C & -7.778522 & -2.515113 & -0.927672 \\
\hline C & -9.250863 & -2.636725 & -0.542786 \\
\hline C & -3.499002 & 1.364347 & -1.147326 \\
\hline C & 3.422337 & -3.464049 & 2.149638 \\
\hline C & 3.205958 & -4.496555 & -0.723507 \\
\hline C & 0.647344 & -3.654563 & 0.785022 \\
\hline C & 1.478336 & 5.061253 & 0.845621 \\
\hline C & 4.153100 & 4.445710 & -0.581802 \\
\hline C & 1.602748 & 4.787214 & -2.223274 \\
\hline C & 4.804970 & -2.796438 & 2.030385 \\
\hline C & 2.673350 & -2.806454 & 3.323601 \\
\hline C & 3.655043 & -4.941979 & 2.514565 \\
\hline 0 & 2.705842 & -1.795795 & -0.123794 \\
\hline 0 & 1.928536 & 2.566130 & -0.333285 \\
\hline 0 & 0.601374 & -2.965370 & -2.324187 \\
\hline 0 & -1.861716 & -2.458372 & -1.621532 \\
\hline 0 & -1.032863 & 3.122767 & -0.475417 \\
\hline 0 & -2.578641 & 2.365396 & 2.027923 \\
\hline 0 & -0.824949 & 2.081462 & 3.508144 \\
\hline $\mathrm{H}$ & 1.603734 & -0.439355 & -2.968083 \\
\hline $\mathrm{H}$ & 3.020032 & -2.269693 & -2.116361 \\
\hline $\mathrm{H}$ & 2.266244 & 1.798430 & -2.228779 \\
\hline $\mathrm{H}$ & 4.357349 & 1.639541 & -0.927460 \\
\hline$H$ & 3.554943 & 0.620957 & 0.273755 \\
\hline$H$ & 3.999103 & -0.102409 & -2.668495 \\
\hline $\mathrm{H}$ & 1.145753 & 0.044524 & -0.001048 \\
\hline $\mathrm{H}$ & 5.529952 & -1.848576 & -1.765933 \\
\hline $\mathrm{H}$ & 6.083340 & -0.236597 & -1.285730 \\
\hline $\mathrm{H}$ & 5.233095 & -1.264291 & -0.117386 \\
\hline $\mathrm{H}$ & -1.411659 & 2.000212 & -2.885863 \\
\hline $\mathrm{H}$ & 0.251445 & 2.522810 & -2.703363 \\
\hline H & -0.112319 & 0.949805 & -3.456811 \\
\hline H & 0.625007 & 1.660057 & 1.465619 \\
\hline H & -0.745567 & 0.550491 & 1.414002 \\
\hline H & -0.904489 & 3.549172 & 2.073969 \\
\hline H & -4.336260 & 3.324887 & 1.735827 \\
\hline H & -3.182148 & 4.235726 & 2.738396 \\
\hline H & -2.932067 & 4.120852 & 0.970271 \\
\hline $\mathrm{H}$ & -2.497929 & 2.739036 & 4.553105 \\
\hline $\mathrm{H}$ & -0.994752 & 2.502364 & 5.479266 \\
\hline $\mathrm{H}$ & -1.158252 & 3.922606 & 4.412012 \\
\hline H & -2.772476 & 0.044472 & 1.188139 \\
\hline $\mathrm{H}$ & -2.767178 & -1.593893 & 0.50893 \\
\hline H & -4.843570 & -1.1388 & 1.6952 \\
\hline
\end{tabular}




\begin{tabular}{rrrr}
$\mathrm{H}$ & -5.154673 & 0.311070 & 0.763066 \\
$\mathrm{H}$ & -5.447116 & -1.067488 & -1.308762 \\
$\mathrm{H}$ & -5.040825 & -2.519613 & -0.405839 \\
$\mathrm{H}$ & -7.047477 & -2.142458 & 1.065680 \\
$\mathrm{H}$ & -7.432823 & -0.685543 & 0.158539 \\
$\mathrm{H}$ & -7.332144 & -3.515339 & -1.002336 \\
$\mathrm{H}$ & -7.694168 & -2.063632 & -1.924932 \\
$\mathrm{H}$ & -9.806774 & -3.248797 & -1.259539 \\
$\mathrm{H}$ & -9.360410 & -3.097375 & 0.445312 \\
$\mathrm{H}$ & -9.727797 & -1.651017 & -0.503885 \\
$\mathrm{H}$ & -3.288094 & 2.020852 & -0.301260 \\
$\mathrm{H}$ & -3.193527 & 1.869109 & -2.065061 \\
$\mathrm{H}$ & -4.584693 & 1.235739 & -1.201794 \\
$\mathrm{H}$ & -3.326815 & -0.681618 & -1.713036 \\
$\mathrm{H}$ & 3.183472 & -5.531258 & -0.363696 \\
$\mathrm{H}$ & 2.653056 & -4.457258 & -1.668284 \\
$\mathrm{H}$ & 4.249539 & -4.239265 & -0.935000 \\
$\mathrm{H}$ & 0.504918 & -4.681248 & 1.142556 \\
$\mathrm{H}$ & 0.212652 & -2.979039 & 1.528597 \\
$\mathrm{H}$ & 0.086560 & -3.553424 & -0.151064 \\
$\mathrm{H}$ & 1.758743 & 4.601522 & 1.799812 \\
$\mathrm{H}$ & 1.769600 & 6.116855 & 0.882152 \\
$\mathrm{H}$ & 0.389523 & 5.012453 & 0.739094 \\
$\mathrm{H}$ & 4.632528 & 4.002911 & -1.462127 \\
$\mathrm{H}$ & 4.379697 & 5.518619 & -0.594647 \\
$\mathrm{H}$ & 4.619229 & 4.018832 & 0.312624 \\
$\mathrm{H}$ & 1.975589 & 4.210021 & -3.077297 \\
$\mathrm{H}$ & 0.508654 & 4.750998 & -2.227043 \\
$\mathrm{H}$ & 1.906312 & 5.828766 & -2.384506 \\
$\mathrm{H}$ & -1.369182 & -3.230084 & -1.950418 \\
$\mathrm{H}$ & 4.682263 & -1.739175 & 1.920673 \\
$\mathrm{H}$ & 5.376366 & -2.999608 & 2.911934 \\
$\mathrm{H}$ & 5.316940 & -3.186673 & 1.175690 \\
$\mathrm{H}$ & 3.035373 & -1.809387 & 3.463938 \\
$\mathrm{H}$ & 1.625788 & -2.778428 & 3.107432 \\
$\mathrm{H}$ & 2.838488 & -3.374649 & 4.215107 \\
$\mathrm{H}$ & 4.719921 & -5.462004 & 2.509947 \\
$\mathrm{H}$ & 4.092890 & -5.386702 & 1.798400 \\
$\mathrm{H}$ & & -5.004104 & 3.488901 \\
$\mathrm{H}$ & & & \\
\hline & & &
\end{tabular}

\section{TBS-TMS}

$\mathrm{HF}=-2439.0730899$ hartrees

$(-1530542.75464315 \mathrm{kcal} / \mathrm{mol})$

$\begin{array}{lrrr}\text { Si } & 2.114381 & 4.166856 & -1.126440 \\ \text { Si } & 2.707495 & -3.122732 & 0.563545 \\ \text { C } & 2.611538 & -1.472522 & -1.631724 \\ \text { C } & 1.190656 & -0.971256 & -1.488138 \\ \text { C } & 0.992502 & 0.382266 & -0.849029 \\ \text { C } & 1.975307 & 1.410591 & -1.454334 \\ \text { C } & 3.406070 & 0.881320 & -1.483189 \\ \text { C } & 3.518990 & -0.421877 & -2.272547 \\ \text { C } & -0.490689 & 0.899861 & -0.863858 \\ \text { C } & 0.155212 & -1.765771 & -1.786309 \\ \text { C } & -1.230488 & -1.437116 & -1.380803 \\ \text { C } & -1.451118 & -0.259902 & -0.437693 \\ \text { C } & 4.960761 & -0.913256 & -2.357697 \\ \text { C } & -0.888634 & 1.419011 & -2.249666 \\ \text { C } & -0.581485 & 2.038399 & 0.176502 \\ \text { C } & -0.080125 & 1.798092 & 1.597696 \\ \text { C } & -1.054747 & 2.278021 & 2.667139 \\ \text { C } & -3.001391 & 1.555934 & 3.775644 \\ \text { C } & 0.173818 & 3.892068 & 3.877710 \\ \text { C } & -2.989100 & -0.021786 & -0.314255 \\ \text { C } & -3.524260 & -1.029898 & 0.724363\end{array}$




\begin{tabular}{|c|c|c|c|}
\hline$C$ & 7 & 1 & \\
\hline c & -5.048137 & -1.083248 & 0.872808 \\
\hline C & -7.295626 & -1.569764 & -0.217749 \\
\hline C & -8.070185 & -1.777319 & -1.518012 \\
\hline C & -9.563269 & -1.995891 & -1.287119 \\
\hline C & -3.484959 & 1.385466 & 0.025021 \\
\hline C & 3.907237 & -3.410650 & 1.982605 \\
\hline C & 2.831380 & -4.527026 & -0.664914 \\
\hline C & 0.966849 & -2.927698 & 1.228040 \\
\hline C & 1.726754 & 5.149097 & 0.414454 \\
\hline C & 3.884276 & 4.455059 & -1.683678 \\
\hline C & 0.954366 & 4.632862 & -2.521936 \\
\hline C & 5.365909 & -3.199108 & 1.536370 \\
\hline C & 3.789010 & -4.864249 & 2.477250 \\
\hline C & 3.621956 & -2.492493 & 3.185602 \\
\hline 0 & 3.116500 & -1.758425 & -0.330569 \\
\hline 0 & 1.898171 & 2.571326 & -0.647906 \\
\hline 0 & 0.297016 & -2.979609 & -2.378760 \\
\hline 0 & -2.118618 & -2.188771 & -1.754959 \\
\hline 0 & -1.016586 & 3.133278 & -0.116973 \\
\hline 0 & -1.919792 & 1.218761 & 2.923678 \\
\hline 0 & -0.399119 & 2.602069 & 3.872183 \\
\hline $\mathrm{H}$ & 2.616052 & -2.383510 & -2.244321 \\
\hline $\mathrm{H}$ & 1.665462 & 1.639392 & -2.487235 \\
\hline $\mathrm{H}$ & 4.056558 & 1.645758 & -1.922890 \\
\hline $\mathrm{H}$ & 3.741981 & 0.717053 & -0.451692 \\
\hline $\mathrm{H}$ & 3.135255 & -0.256047 & -3.290409 \\
\hline $\mathrm{H}$ & 1.319300 & 0.273937 & 0.196191 \\
\hline $\mathrm{H}$ & -1.087008 & -0.625843 & 0.538416 \\
\hline $\mathrm{H}$ & 5.025322 & -1.859665 & -2.905922 \\
\hline $\mathrm{H}$ & 5.591562 & -0.181223 & -2.871773 \\
\hline $\mathrm{H}$ & 5.366311 & -1.074060 & -1.354550 \\
\hline $\mathrm{H}$ & -1.965808 & 1.586129 & -2.313499 \\
\hline $\mathrm{H}$ & -0.403183 & 2.370024 & -2.469840 \\
\hline $\mathrm{H}$ & -0.613816 & 0.697719 & -3.026384 \\
\hline $\mathrm{H}$ & 0.854467 & 2.367511 & 1.663377 \\
\hline $\mathrm{H}$ & 0.150767 & 0.752559 & 1.814382 \\
\hline $\mathrm{H}$ & -1.616629 & 3.164188 & 2.317710 \\
\hline $\mathrm{H}$ & -3.649344 & 0.678679 & 3.826193 \\
\hline $\mathrm{H}$ & -2.647809 & 1.808167 & 4.779854 \\
\hline $\mathrm{H}$ & -3.571885 & 2.402138 & 3.366952 \\
\hline $\mathrm{H}$ & -0.571225 & 4.661139 & 3.630242 \\
\hline $\mathrm{H}$ & 0.549643 & 4.069615 & 4.886722 \\
\hline $\mathrm{H}$ & 1.011042 & 3.973455 & 3.171979 \\
\hline $\mathrm{H}$ & -3.076582 & -0.771750 & 1.694276 \\
\hline $\mathrm{H}$ & -3.166813 & -2.032341 & 0.460775 \\
\hline $\mathrm{H}$ & -5.291791 & -1.879081 & 1.589341 \\
\hline $\mathrm{H}$ & -5.421695 & -0.153028 & 1.321301 \\
\hline $\mathrm{H}$ & 660504 & -0.506636 & -1.122458 \\
\hline $\mathrm{H}$ & -5.363951 & -2.227772 & -0.929768 \\
\hline $\mathrm{H}$ & -7.445191 & -2.440691 & 0.436288 \\
\hline $\mathrm{H}$ & -7.716002 & -0.706363 & 0.318388 \\
\hline $\mathrm{H}$ & -7.649640 & -2.637707 & -2.054724 \\
\hline $\mathrm{H}$ & -7.921853 & -0.904904 & -2.167810 \\
\hline $\mathrm{H}$ & -10.100324 & -2.141306 & -2.229430 \\
\hline $\mathrm{H}$ & -9.738400 & -2.879376 & -0.663346 \\
\hline $\mathrm{H}$ & -10.011017 & -1.135889 & -0.776937 \\
\hline $\mathrm{H}$ & -3.184395 & 1.685109 & 1.032514 \\
\hline $\mathrm{H}$ & -3.144764 & 2.147922 & -0.675447 \\
\hline $\mathrm{H}$ & -4.579255 & 1.385988 & -0.006501 \\
\hline $\mathrm{H}$ & -3.413387 & -0.285556 & -1.289988 \\
\hline $\mathrm{H}$ & 2.558300 & -5.480711 & -0.199681 \\
\hline $\mathrm{H}$ & 2.163464 & -4.361933 & -1.516636 \\
\hline $\mathrm{H}$ & 3.8515 & -4.622010 & -1.05180 \\
\hline
\end{tabular}




$\begin{array}{rrrr}\mathrm{H} & 0.643954 & -3.847408 & 1.729590 \\ \mathrm{H} & 0.909470 & -2.114272 & 1.959424 \\ \mathrm{H} & 0.254226 & -2.718727 & 0.422142 \\ \mathrm{H} & 2.418150 & 4.898709 & 1.226337 \\ \mathrm{H} & 1.809459 & 6.225417 & 0.226094 \\ \mathrm{H} & 0.701485 & 4.939868 & 0.740176 \\ \mathrm{H} & 4.102087 & 3.966025 & -2.639700 \\ \mathrm{H} & 4.057955 & 5.528991 & -1.823574 \\ \mathrm{H} & 4.605518 & 4.095582 & -0.941926 \\ \mathrm{H} & 1.081721 & 4.007034 & -3.412604 \\ \mathrm{H} & -0.088711 & 4.572669 & -2.195572 \\ \mathrm{H} & 1.153715 & 5.667727 & -2.826445 \\ \mathrm{H} & -0.600523 & -3.339244 & -2.487049 \\ \mathrm{H} & 5.386539 & -2.569794 & 0.671246 \\ \mathrm{H} & 5.917345 & -2.735799 & 2.327675 \\ \mathrm{H} & 5.807336 & -4.144750 & 1.300143 \\ \mathrm{H} & 2.762469 & -5.090712 & 2.676817 \\ \mathrm{H} & 4.161071 & -5.528281 & 1.725231 \\ \mathrm{H} & 4.361347 & -4.983720 & 3.373385 \\ \mathrm{H} & 4.352588 & -1.711510 & 3.219382 \\ \mathrm{H} & 2.646630 & -2.064316 & 3.084097 \\ \mathrm{H} & 3.668434 & -3.063715 & 4.089176\end{array}$

\section{Full Gaussian09 Citation:}

Gaussian 09, Revision D.01, Frisch, M. J.; Trucks, G. W.; Schlegel, H. B.; Scuseria, G. E.; Robb, M. A.; Cheeseman, J. R.; Scalmani, G.; Barone, V.; Mennucci, B.; Petersson, G. A.; Nakatsuji, H.; Caricato, M.; Li, X.; Hratchian, H. P.; Izmaylov, A. F.; Bloino, J.; Zheng, G.; Sonnenberg, J. L.; Hada, M.; Ehara, M.; Toyota, K.; Fukuda, R.; Hasegawa, J.; Ishida, M.; Nakajima, T.; Honda, Y.; Kitao, O.; Nakai, H.; Vreven, T.; Montgomery, J. A., Jr.; Peralta, J. E.; Ogliaro, F.; Bearpark, M.; Heyd, J. J.; Brothers, E.; Kudin, K. N.; Staroverov, V. N.; Kobayashi, R.; Normand, J.; Raghavachari, K.; Rendell, A.; Burant, J. C.; Iyengar, S. S.; Tomasi, J.; Cossi, M.; Rega, N.; Millam, N. J.; Klene, M.; Knox, J. E.; Cross, J. B.; Bakken, V.; Adamo, C.; Jaramillo, J.; Gomperts, R.; Stratmann, R. E.; Yazyev, O.; Austin, A. J.; Cammi, R.; Pomelli, C.; Ochterski, J. W.; Martin, R. L.; Morokuma, K.; Zakrzewski, V. G.; Voth, G. A.; Salvador, P.; Dannenberg, J. J.; Dapprich, S.; Daniels, A. D.; Farkas, Ö.; Foresman, J. B.; Ortiz, J. V.; Cioslowski, J.; Fox, D. J. Gaussian, Inc., Wallingford CT, 2009. 